\title{
$0 T-8$
}

GROUND WATER IN THE VICINITY OF MCGREGOR

RANGE CAMP SITE, OTERO COUNTY, N. MEX.

By

.

James W. Hood

OFR: $58-49$ 


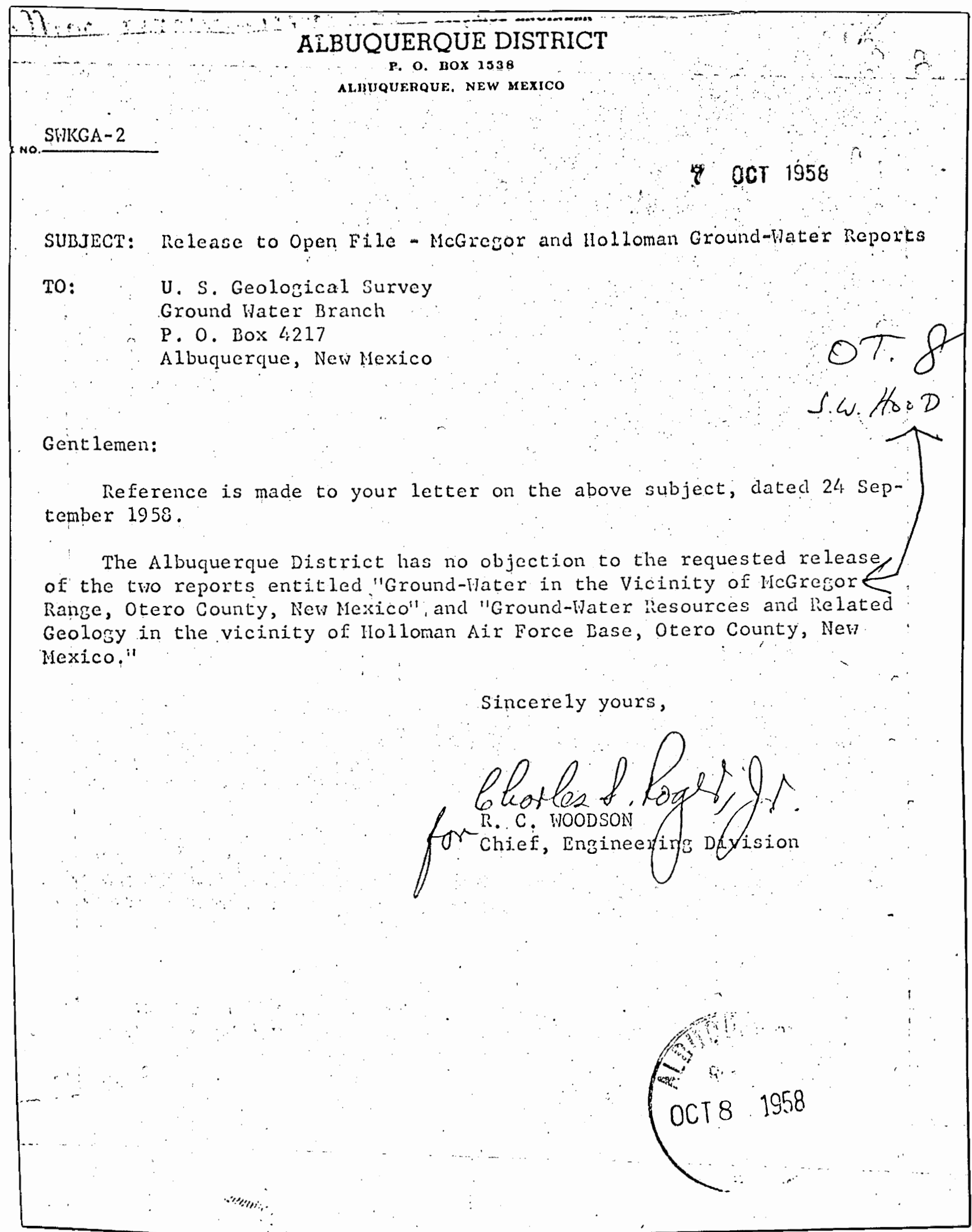




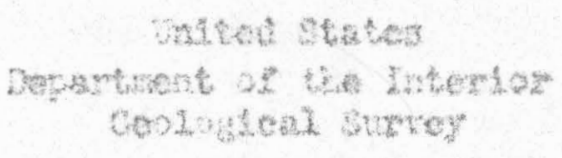

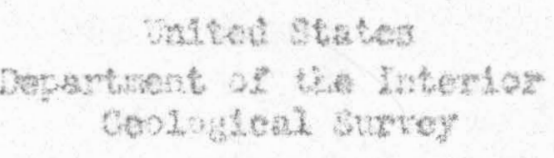

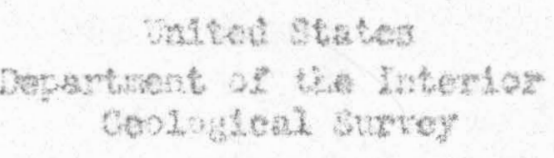

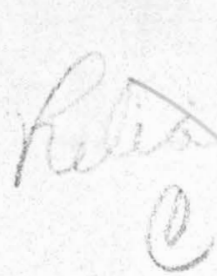
$\frac{3}{8}$
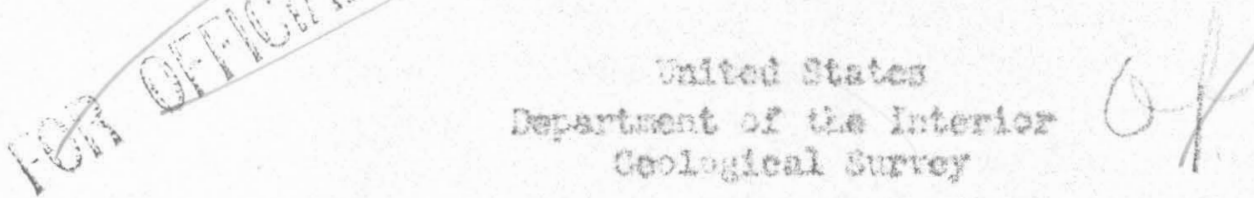

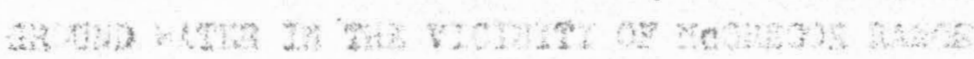

$$
\begin{aligned}
& \text { Is }
\end{aligned}
$$

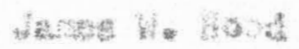

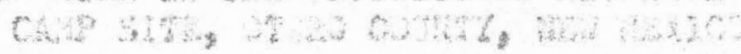

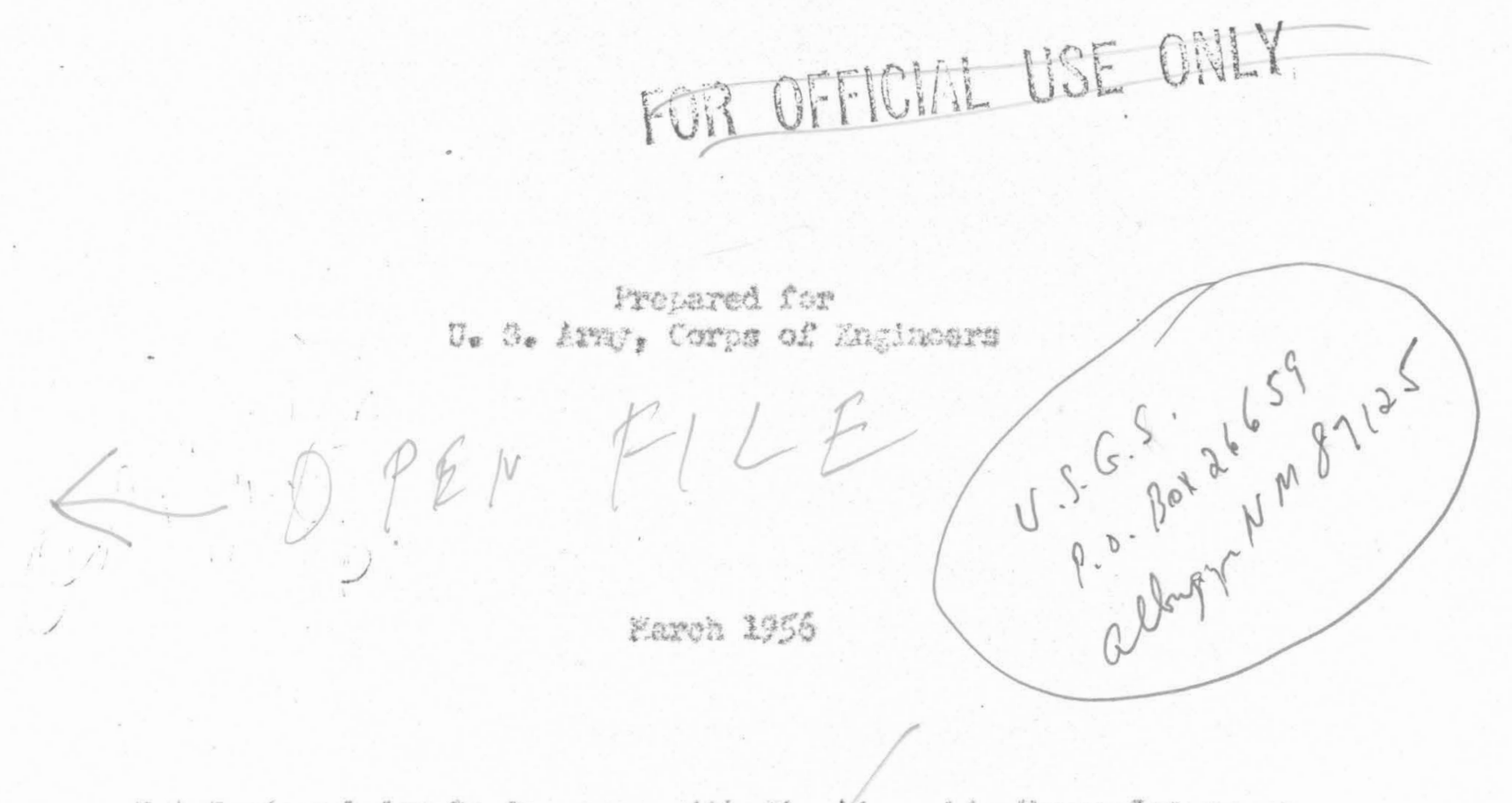

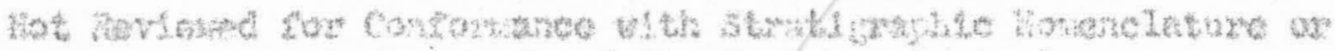

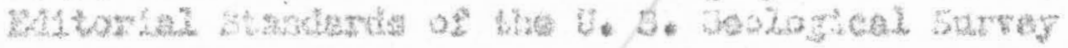




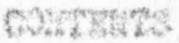

12.4. pata

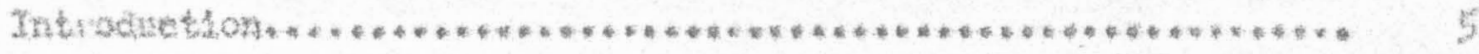

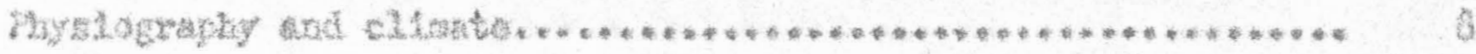

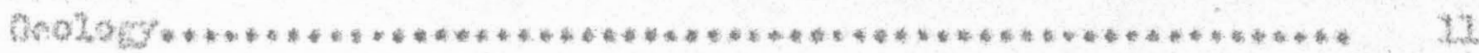

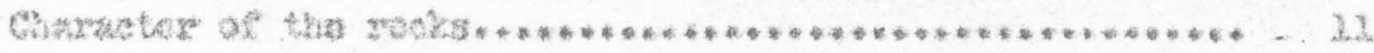

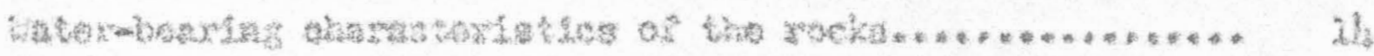

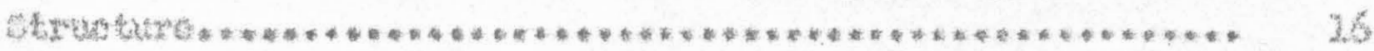

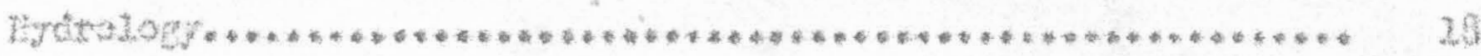

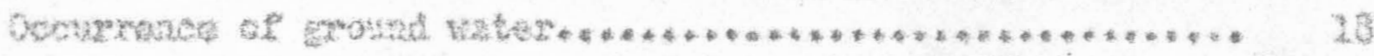

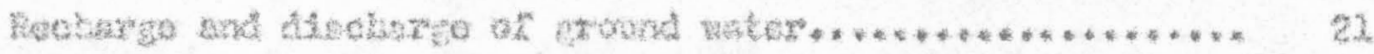

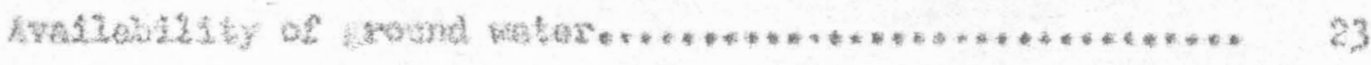

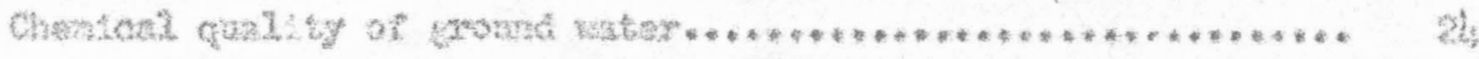

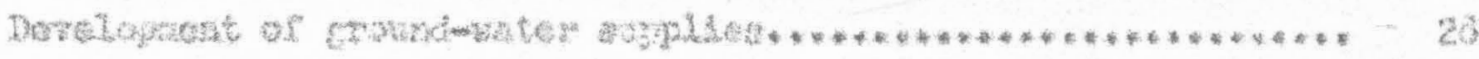

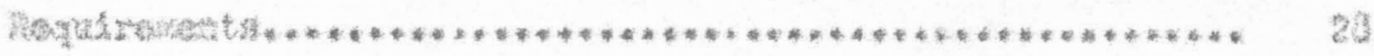

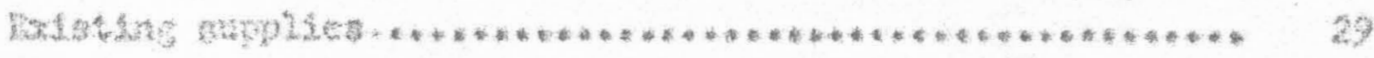

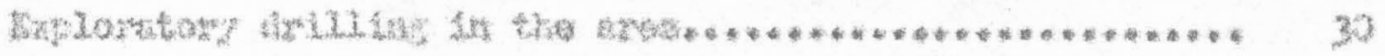

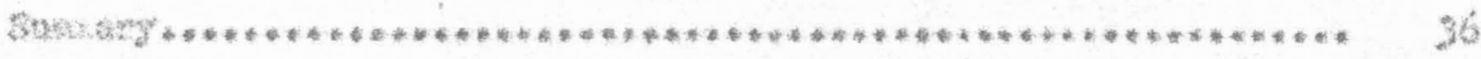

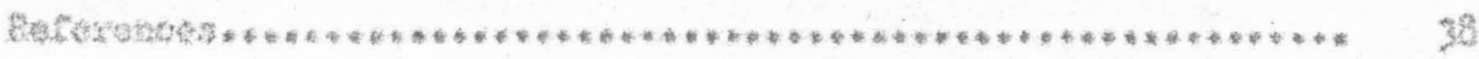




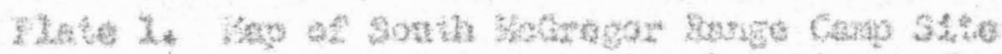

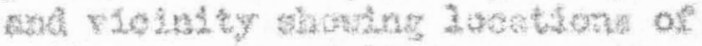

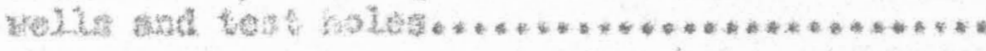

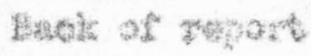

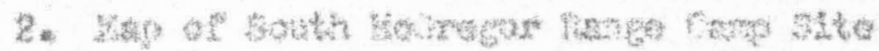

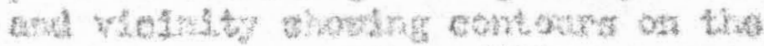

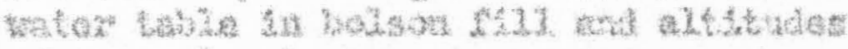

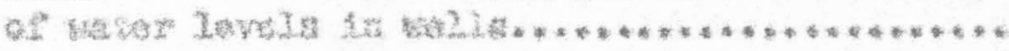

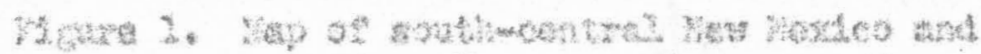

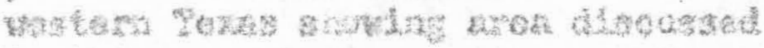

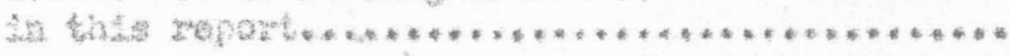

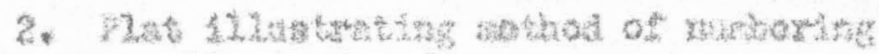

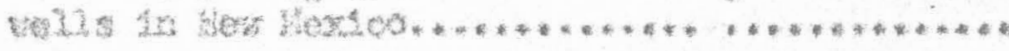




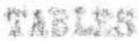

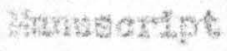
gotedes

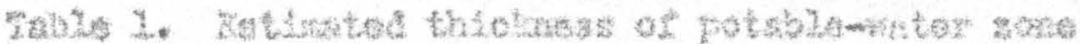

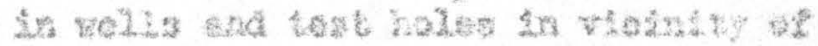

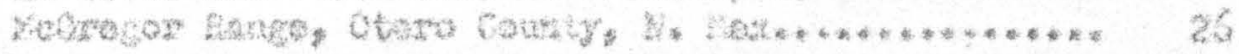

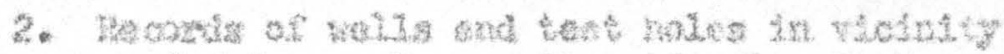

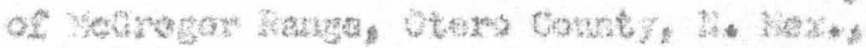

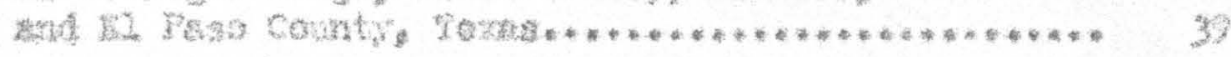

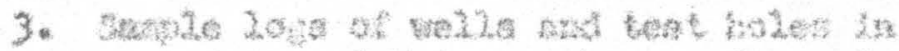

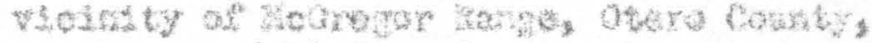

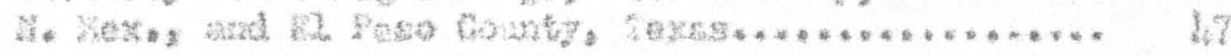

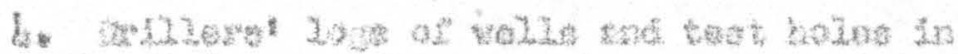

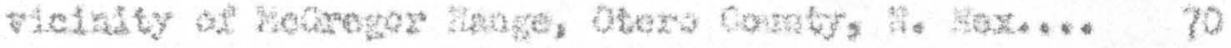

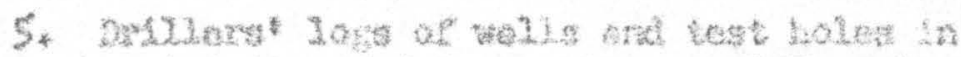

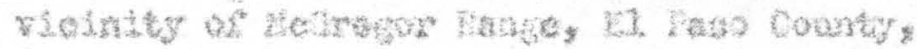

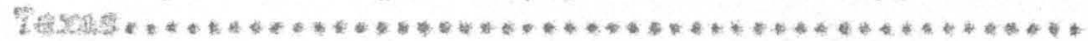

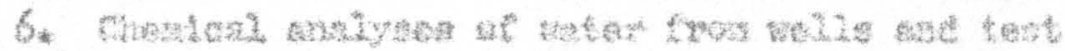

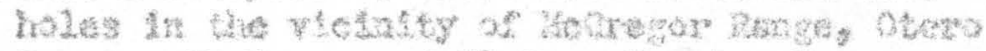

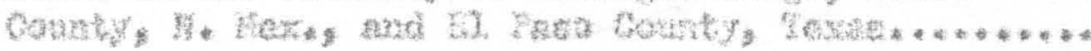




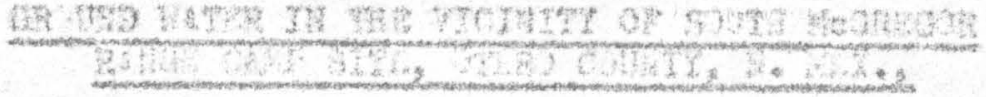 \\ is \\ Jande the tod

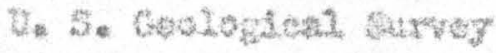 \\ Warch 2356}

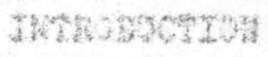

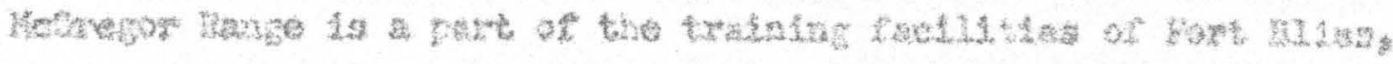
Ior

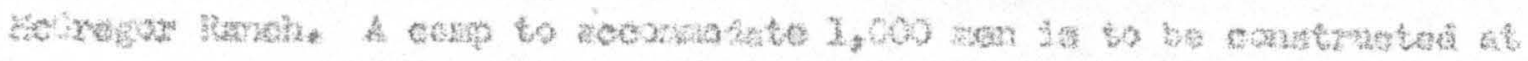

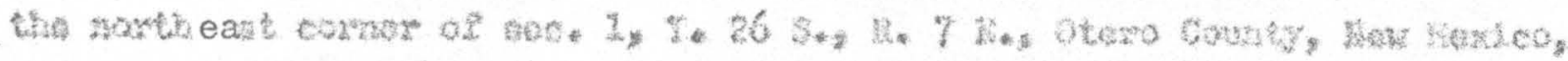

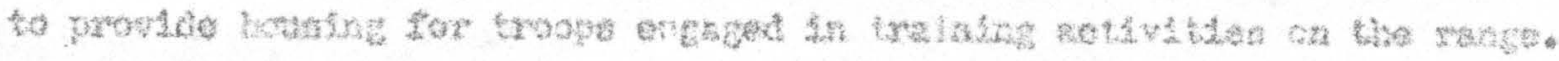

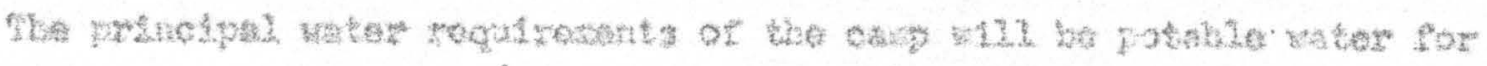

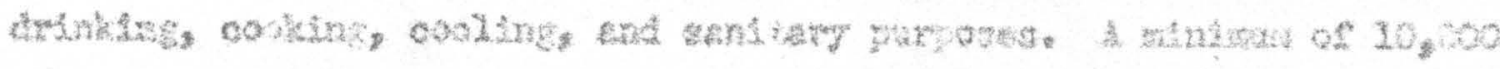

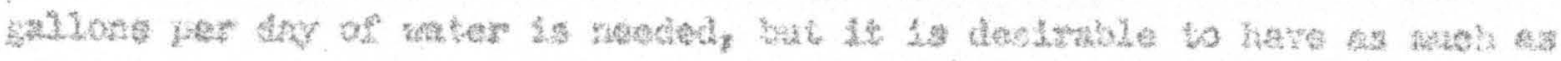

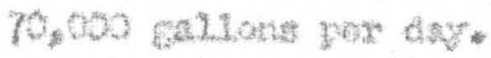

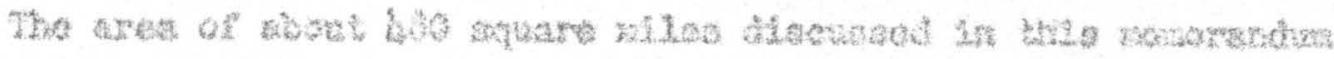

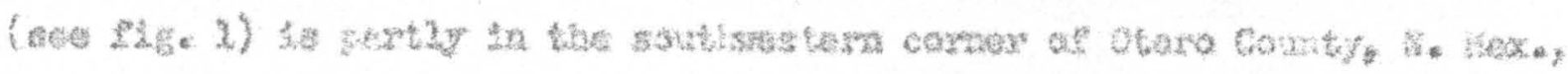

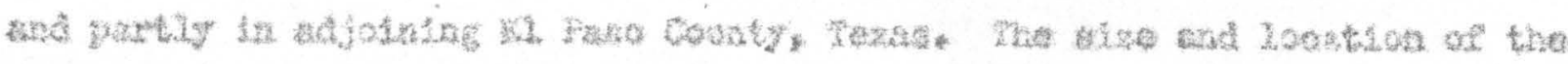

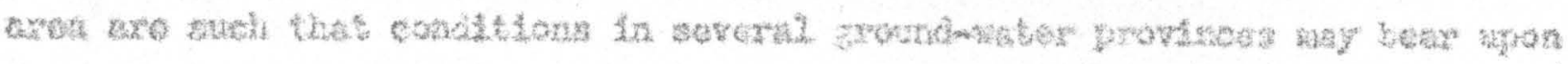

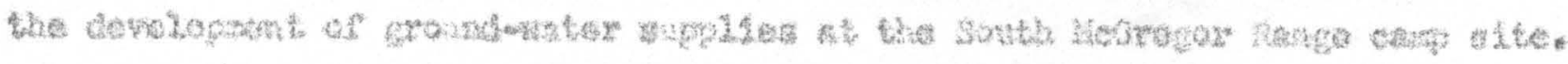

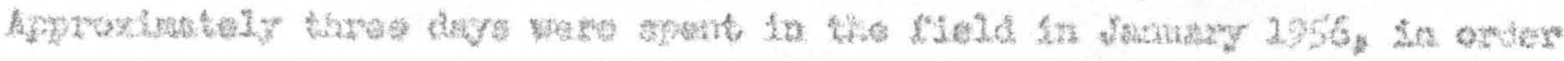

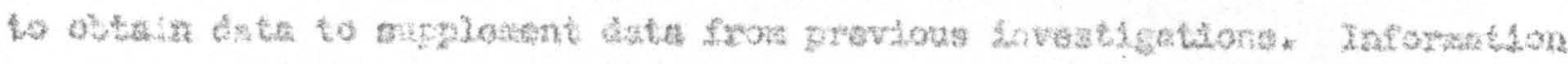

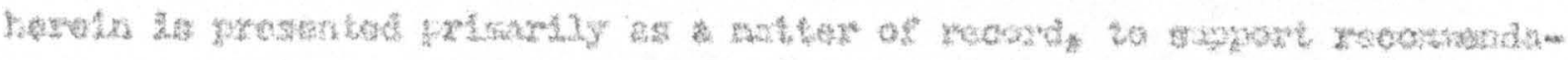

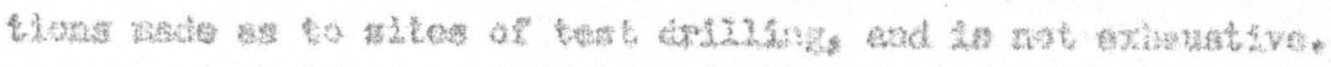




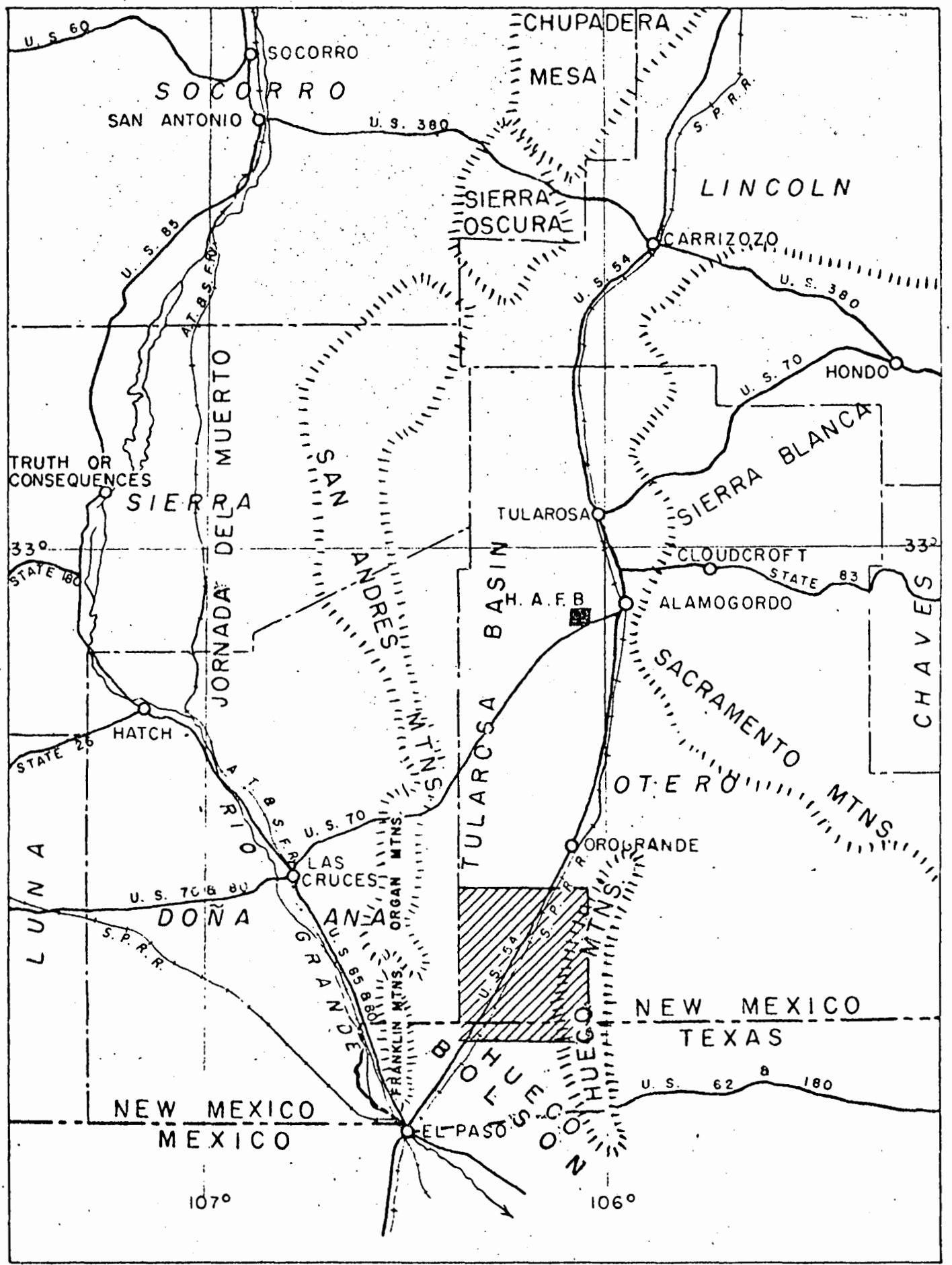

Fig. 1.-Map of south-central New Mexico and western Texas showing area discussed in this report. 


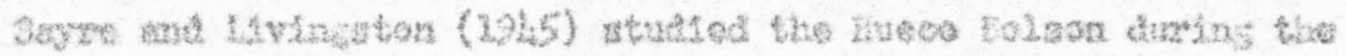

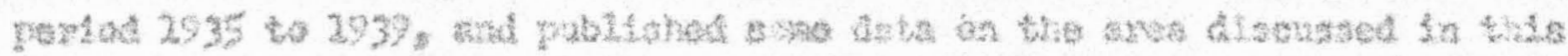

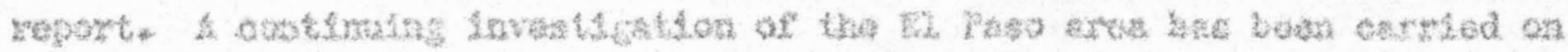

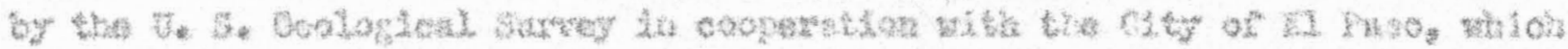

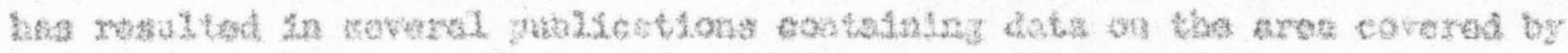

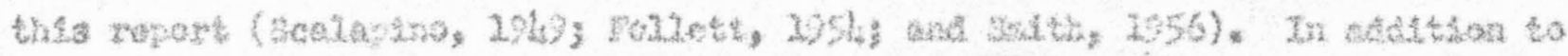

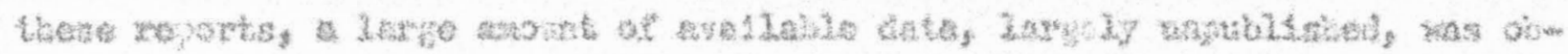

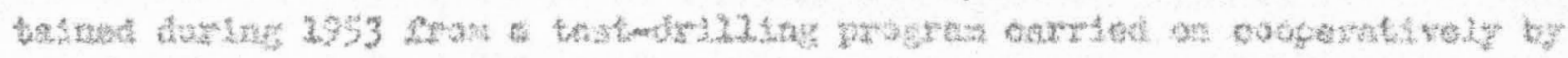

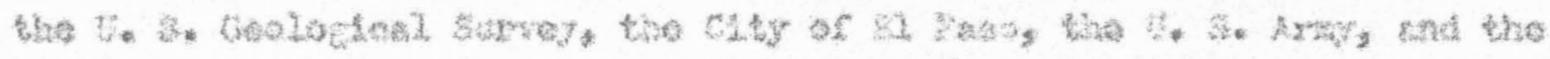

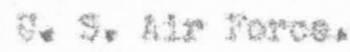

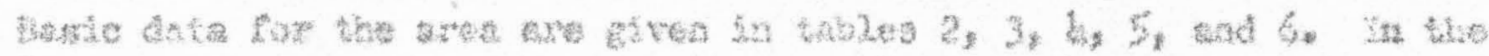

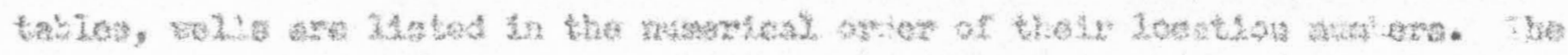

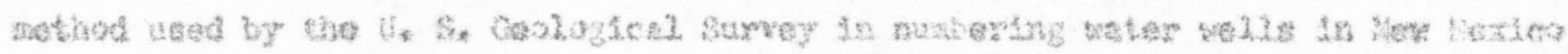

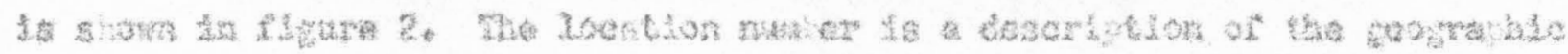

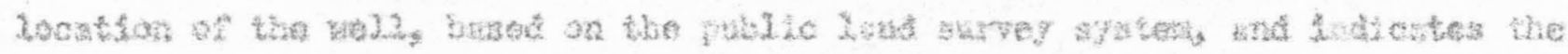

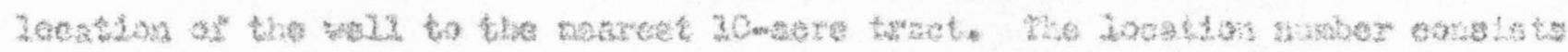

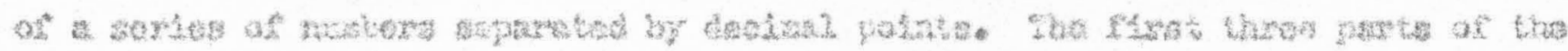

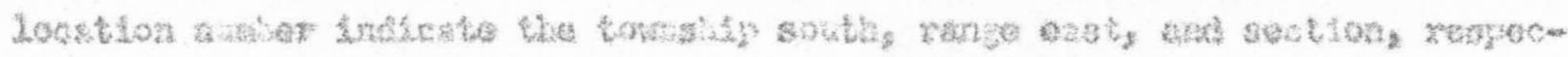

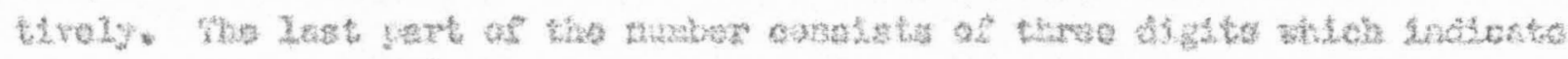

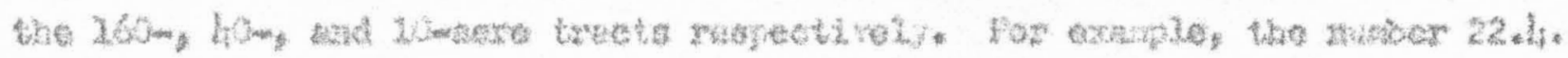

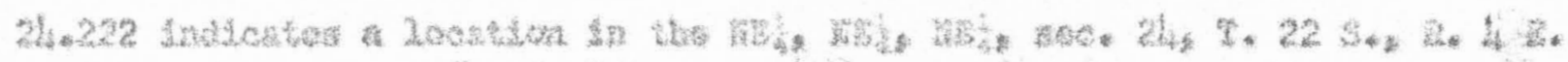

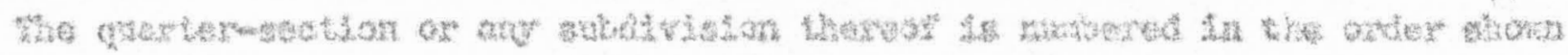

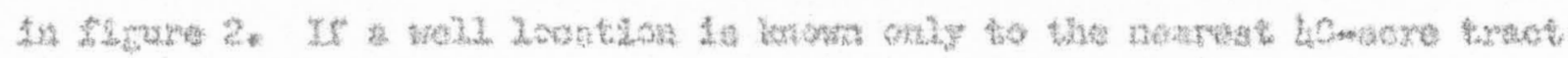

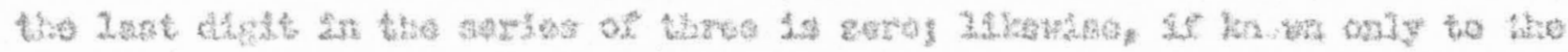

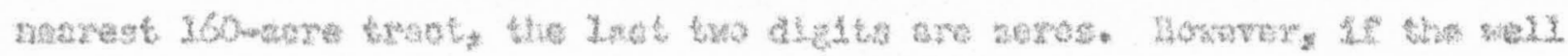

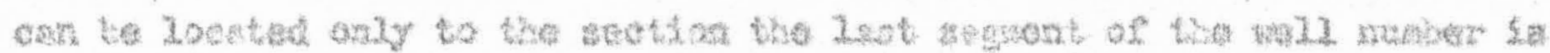




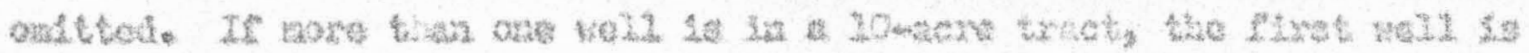

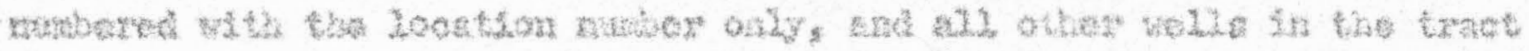

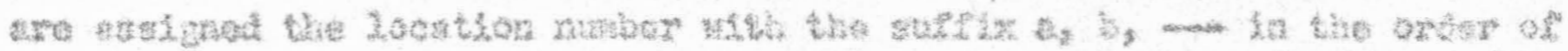

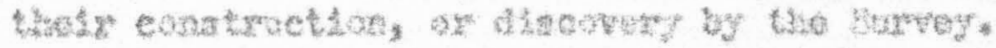

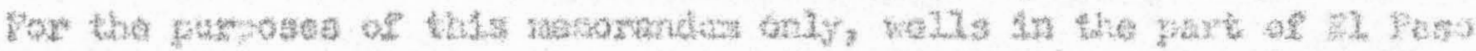

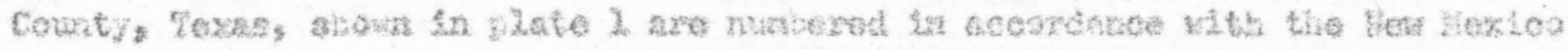

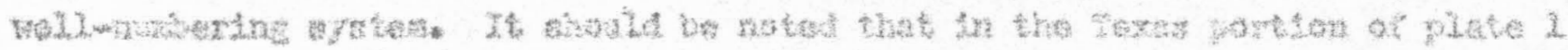

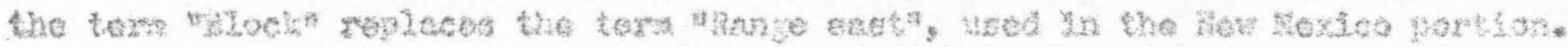

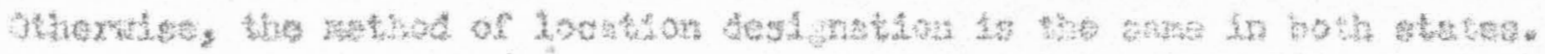




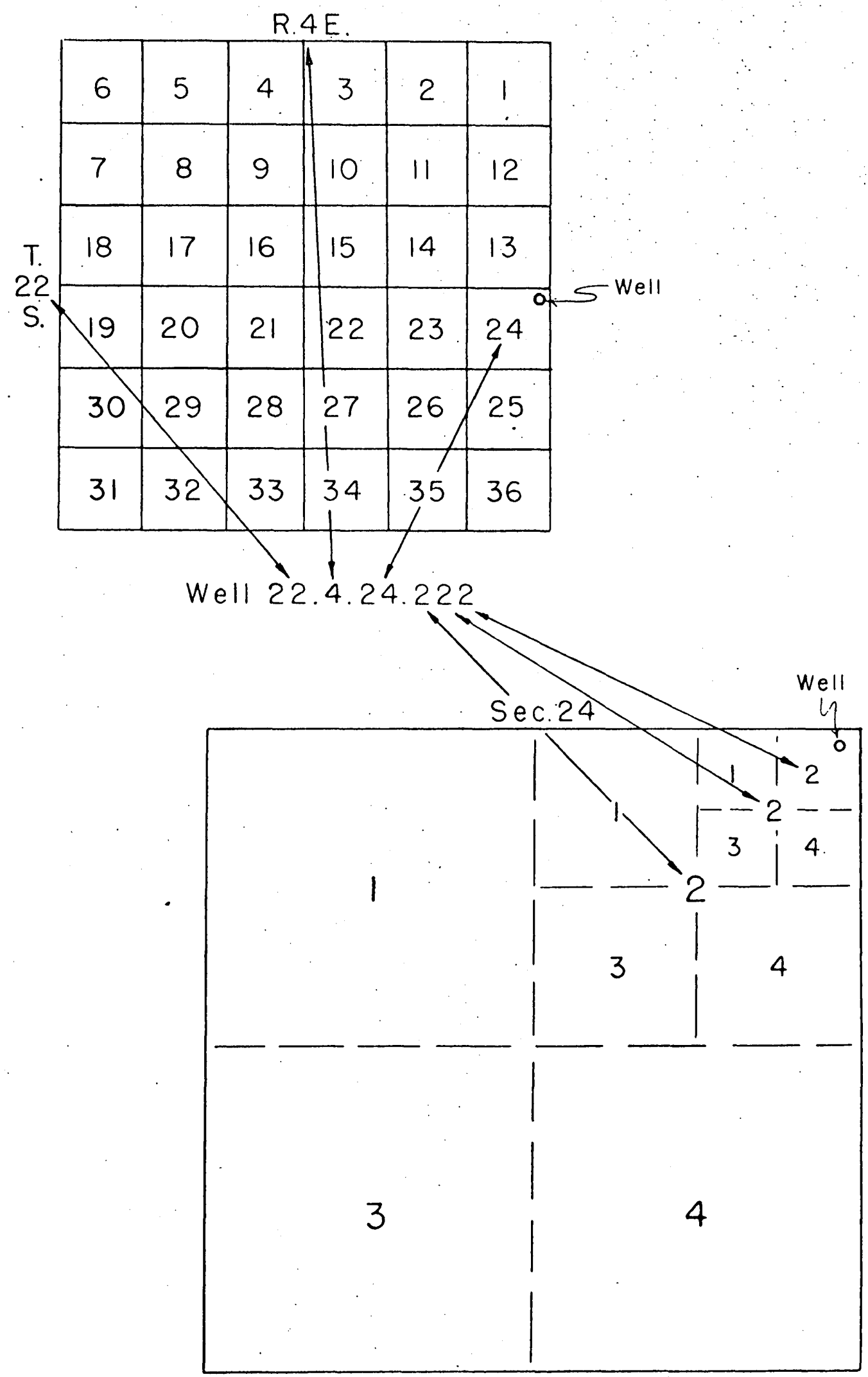

Figure 2--Plat illustrating method of numbering wells in New Mexico 


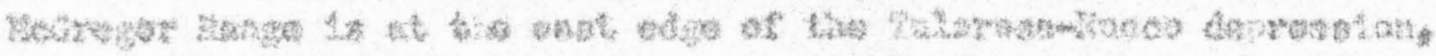

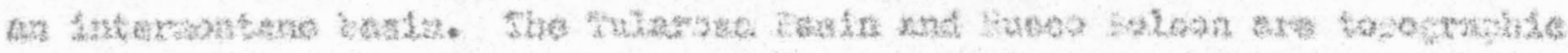
guthtys

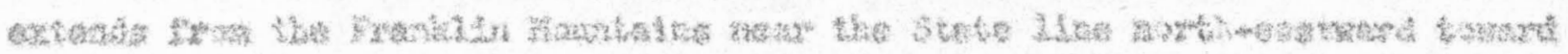

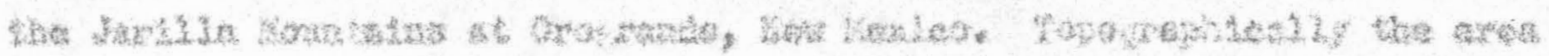

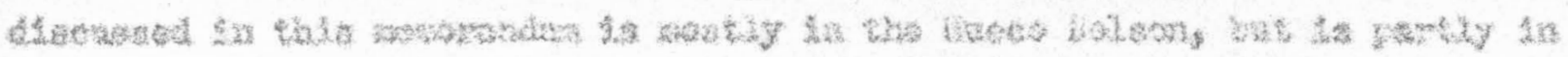

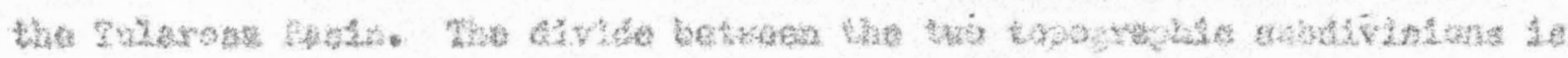

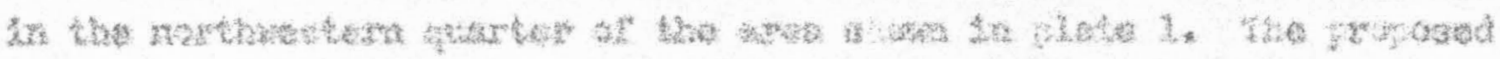

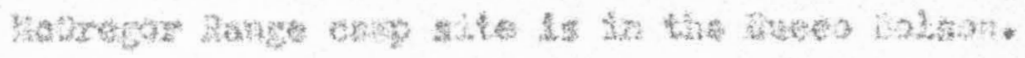

ond a

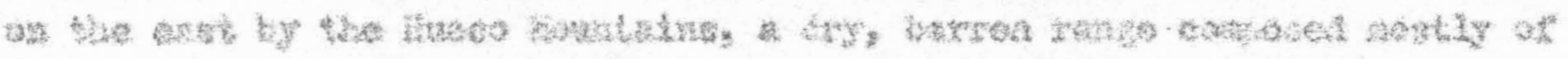

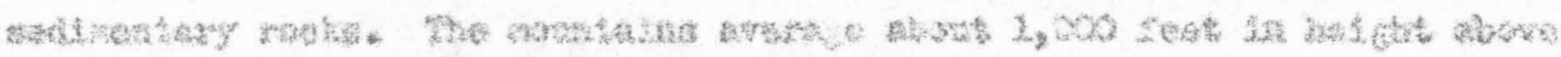
s.

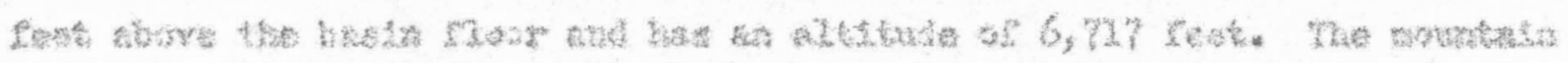

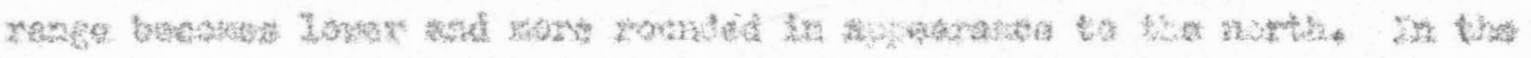

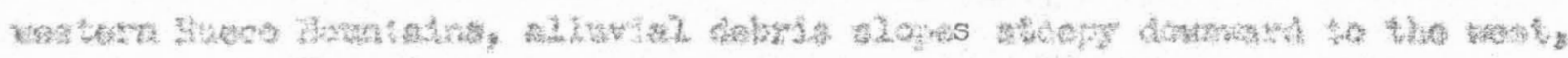

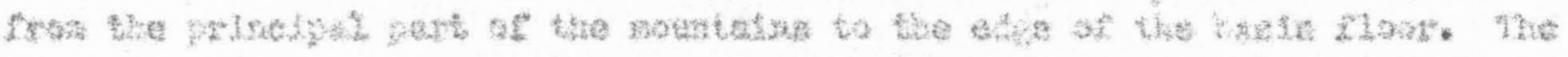

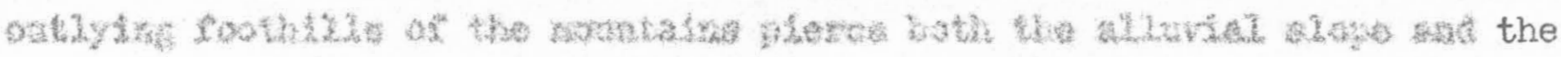

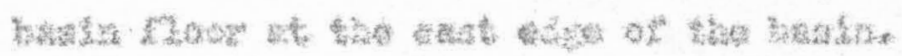

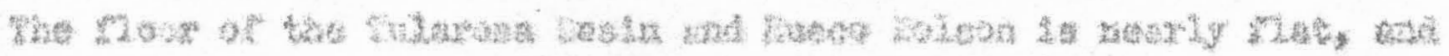

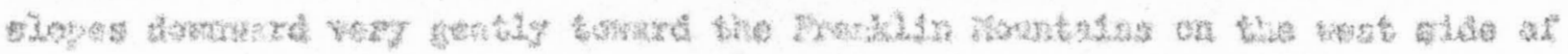

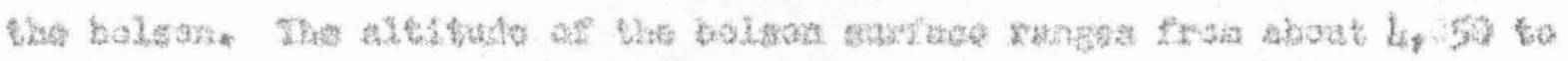

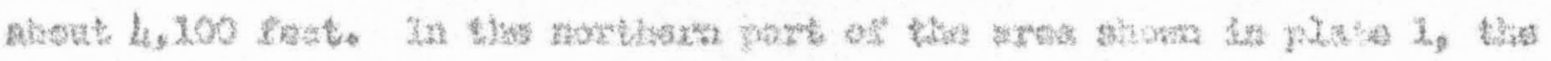

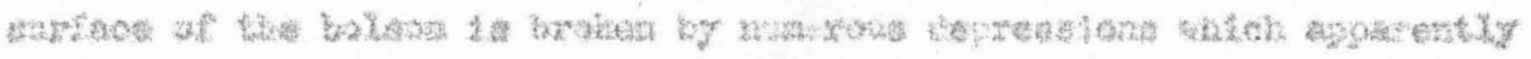




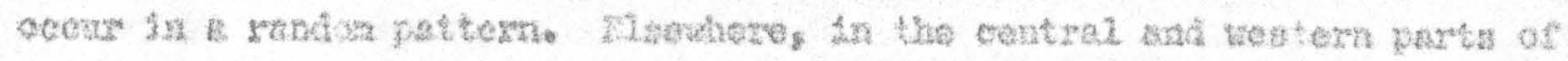

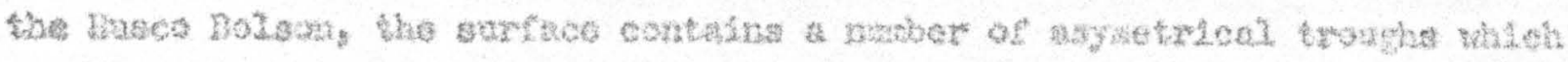

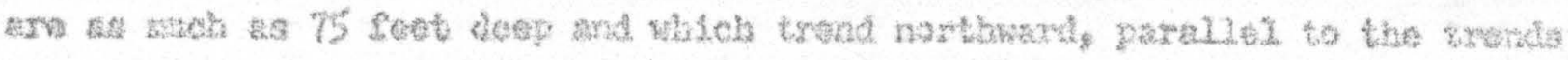

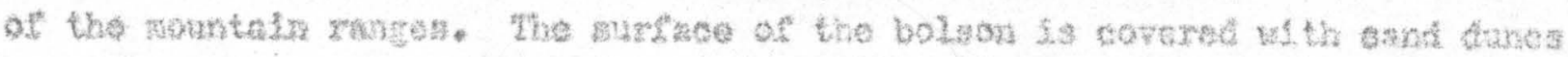

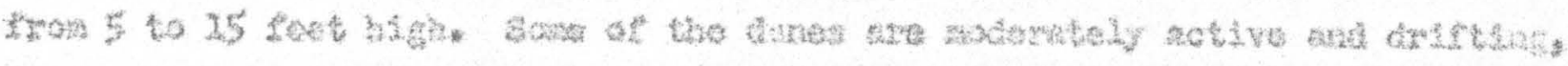

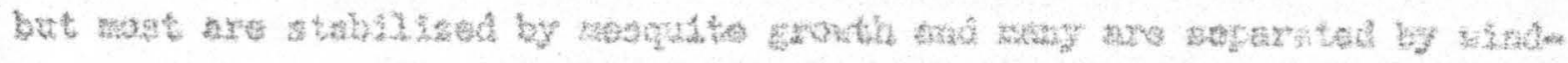

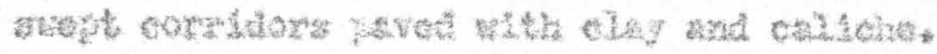

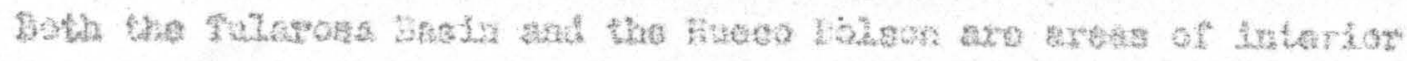

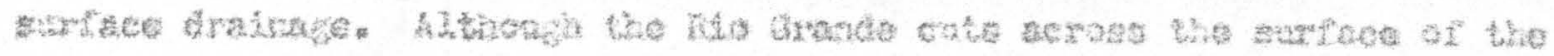

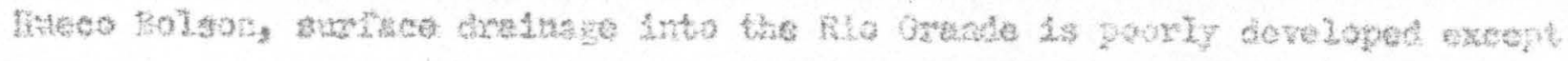

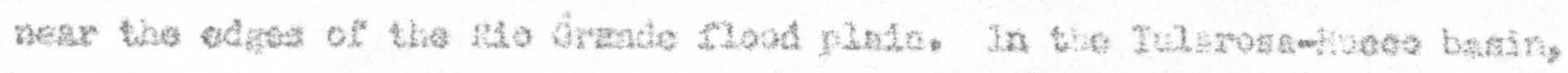

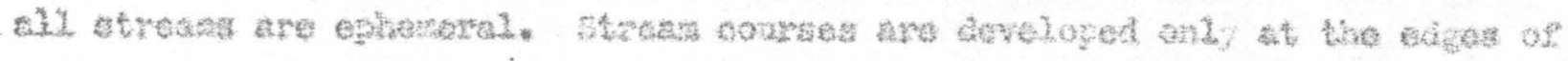

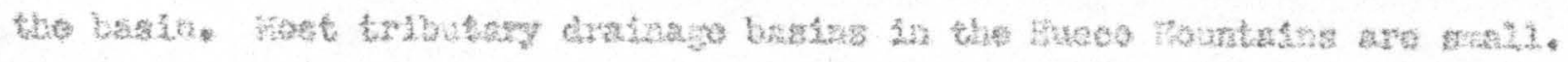

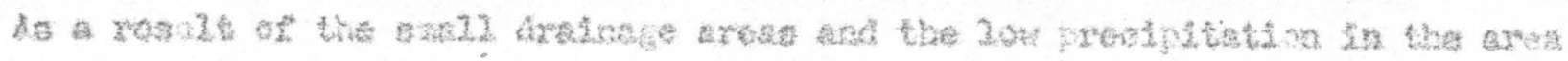

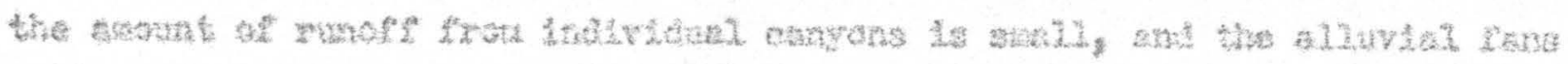

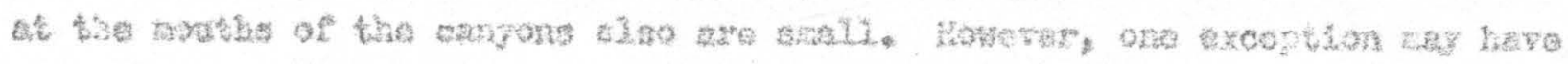

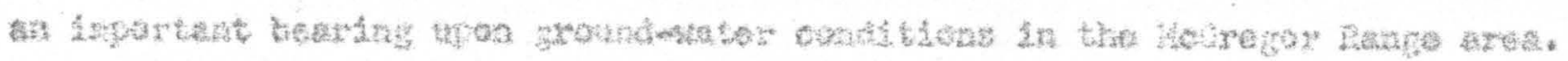

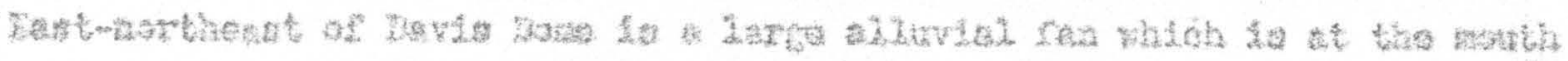

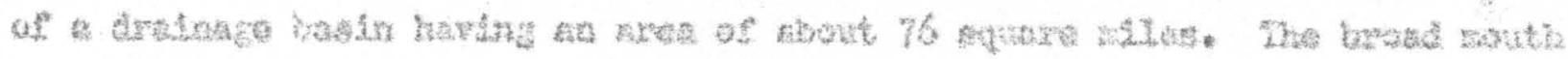

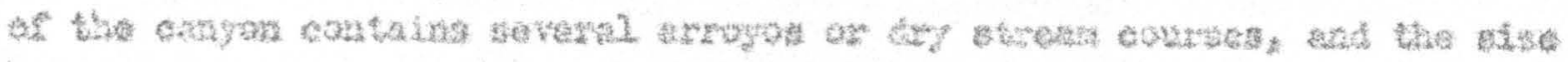

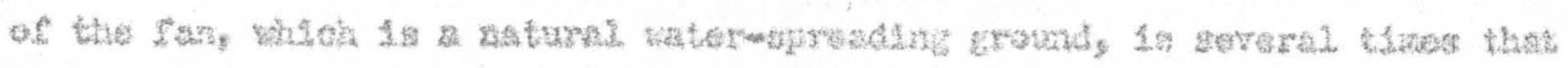

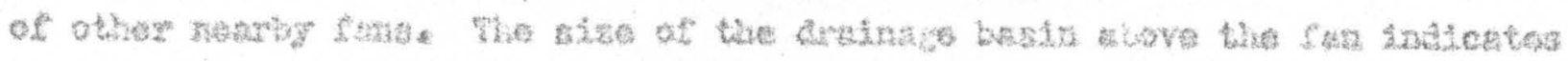

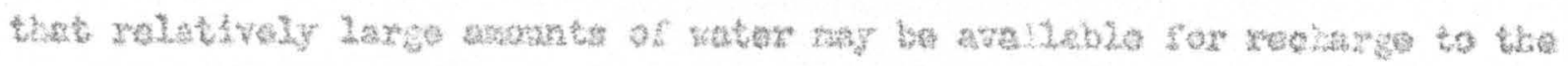

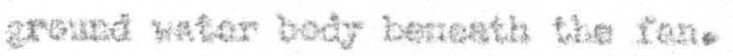




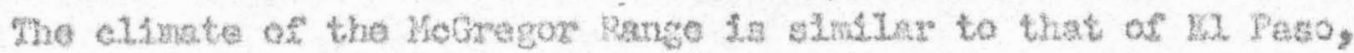

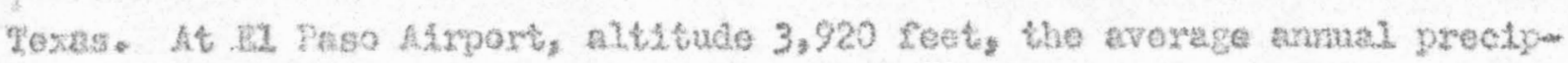
Station fo 7.03 inchers, elthough prociptheton in 1953 was andy 4.42 inches.

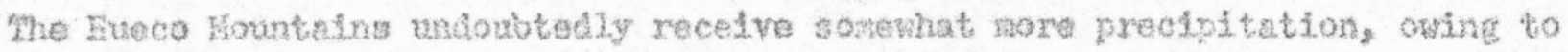

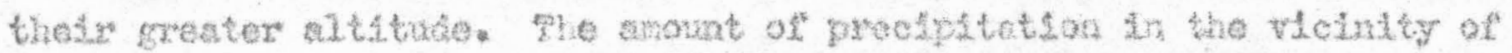

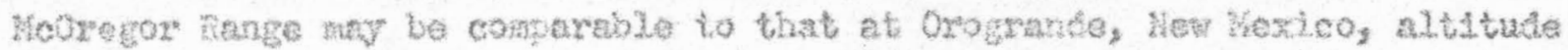

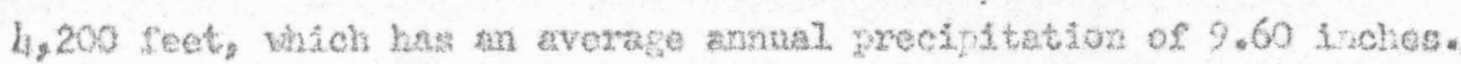

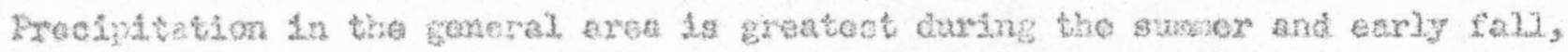

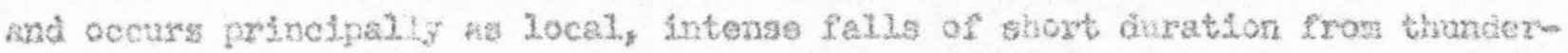
gtoriss:

At El Pago the dverage emmal tenperature is $63.3^{\circ}$. 2ho highest strerage nonthiy temprature of $31.3^{\circ} \%$ ocoure in July and tho lowest averase

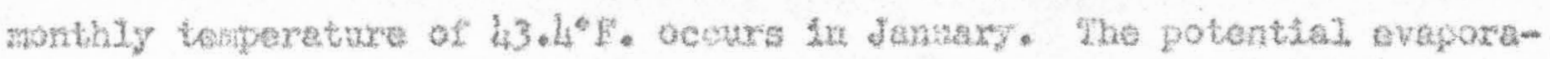

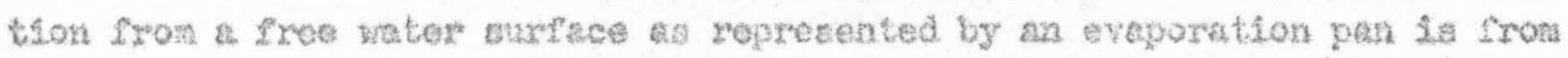
3 so 20 seet jer yer. 
Wrots?

Derecter of tho gaks

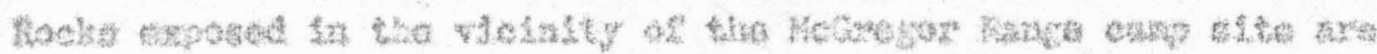

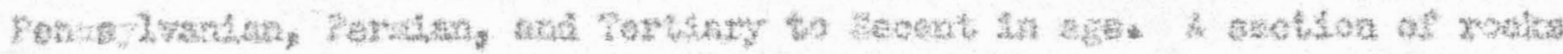

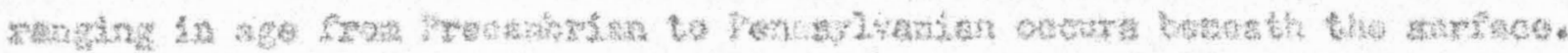

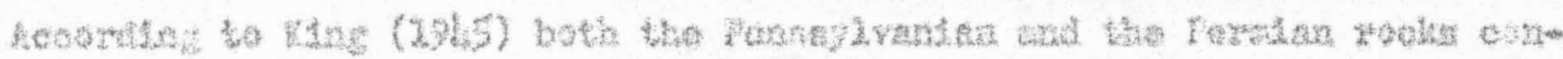

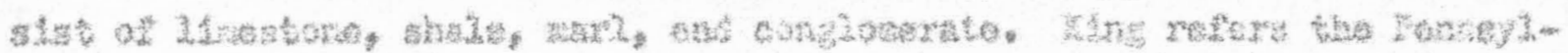

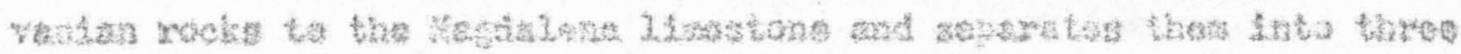
4.

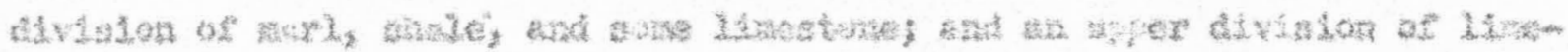

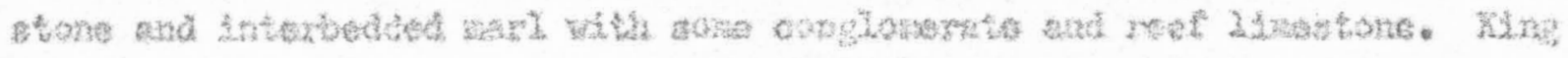

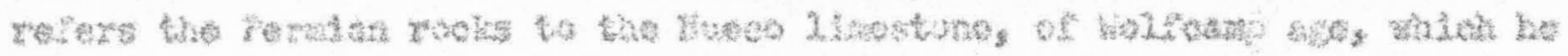

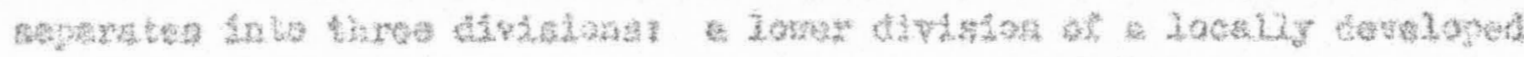

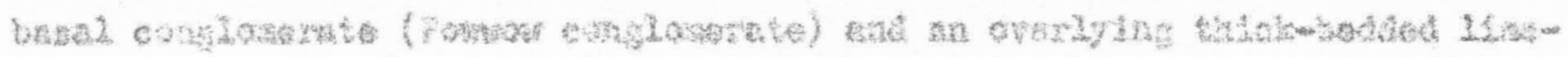

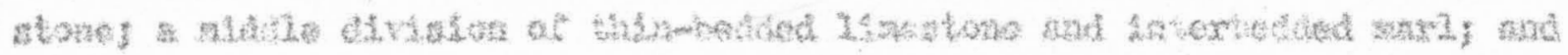

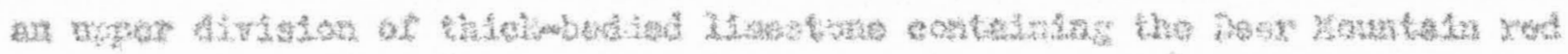

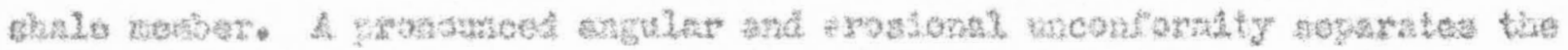

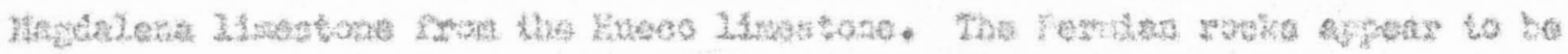

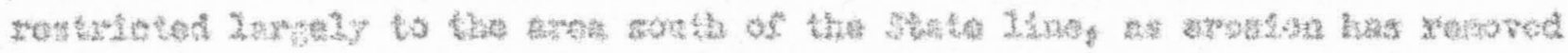

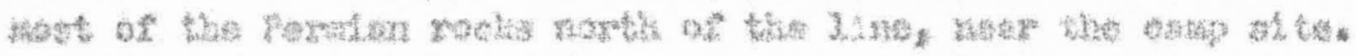

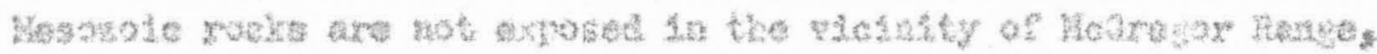

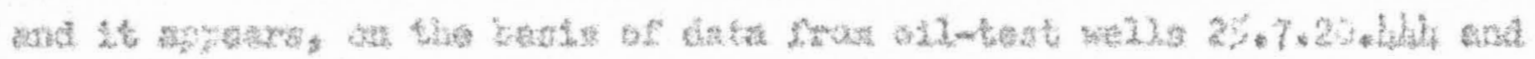

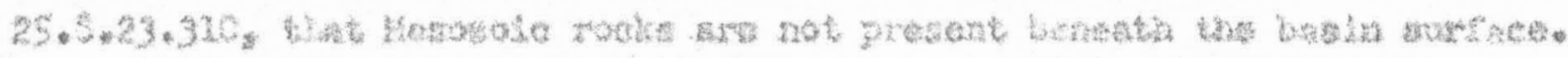




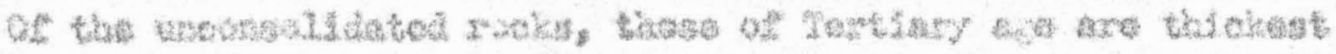

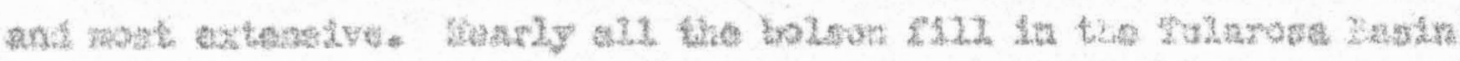

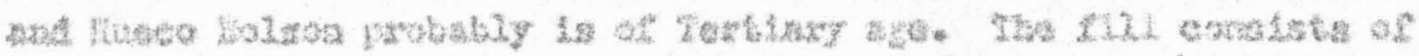

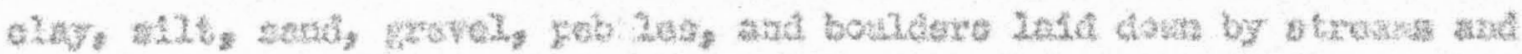

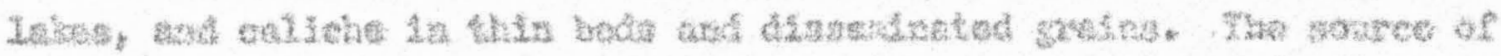

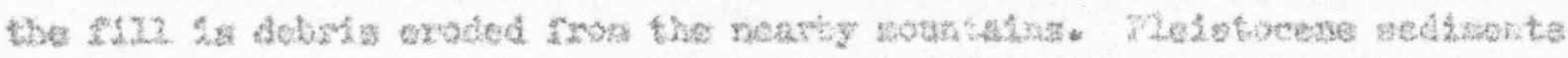

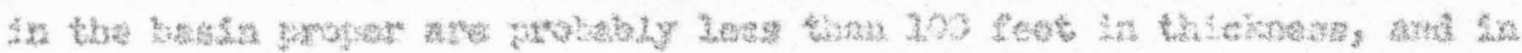

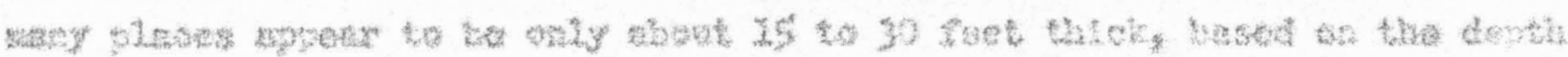

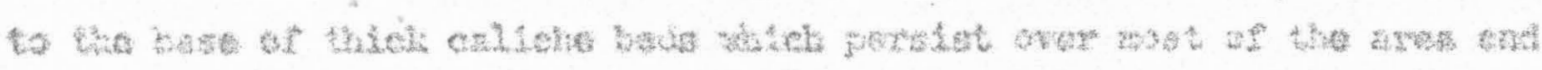

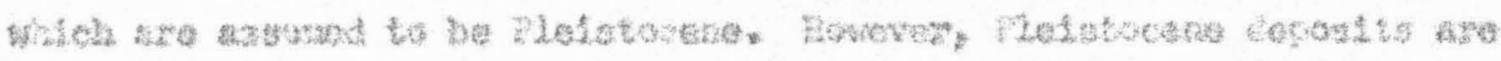

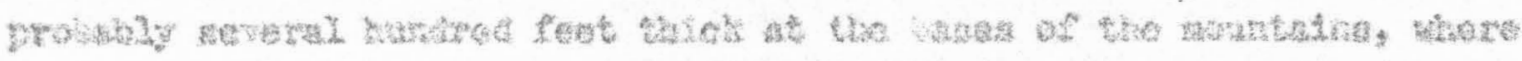

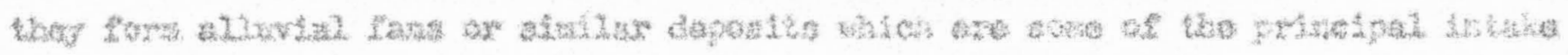

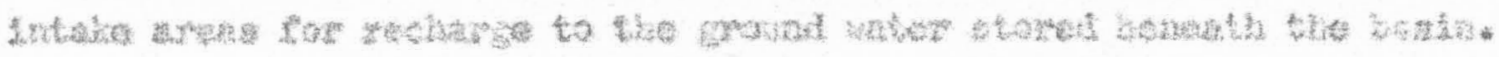

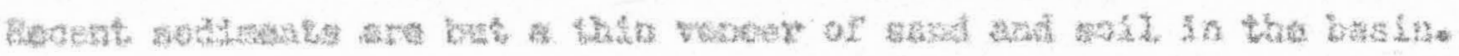




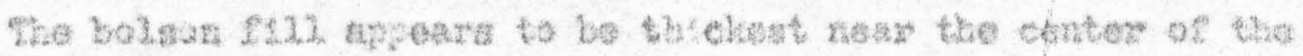

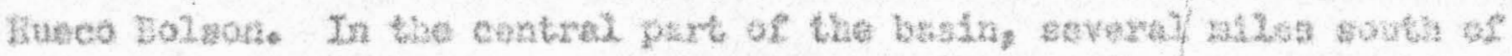

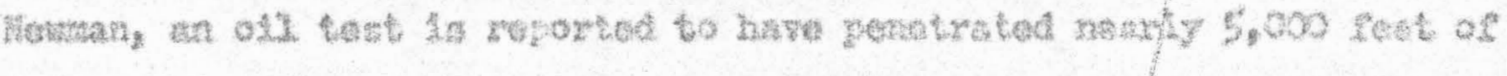

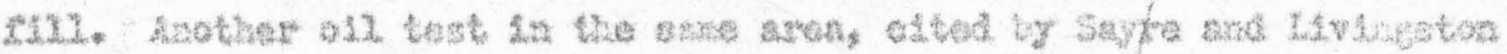

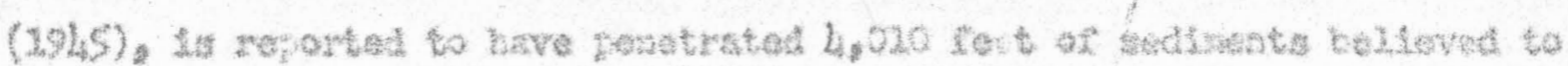

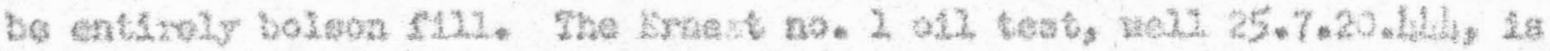

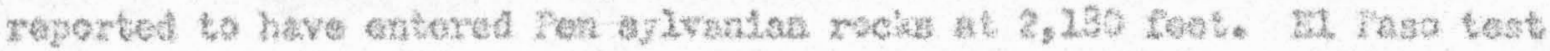

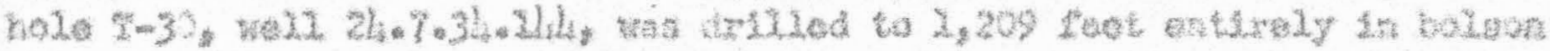

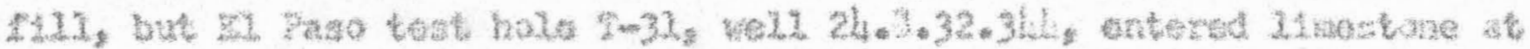

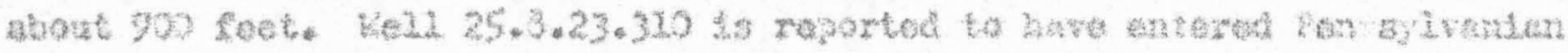

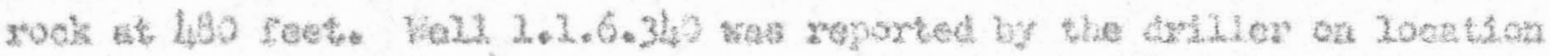

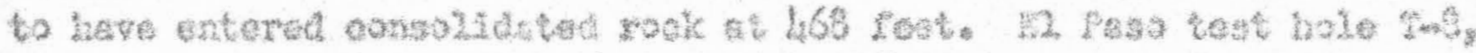

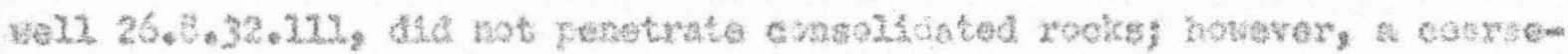

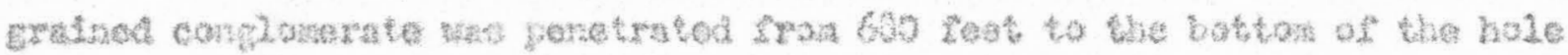

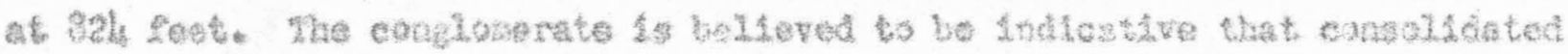

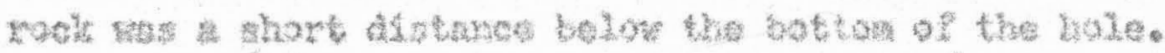

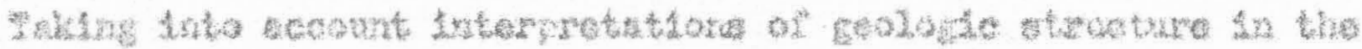

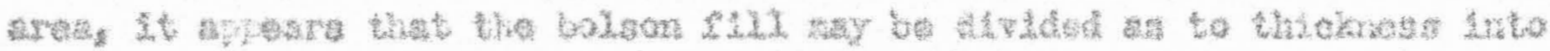

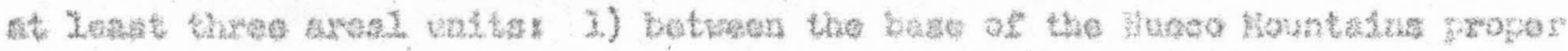

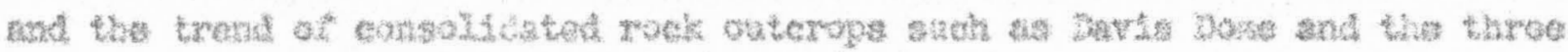

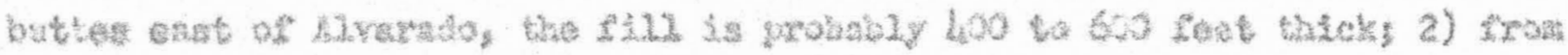

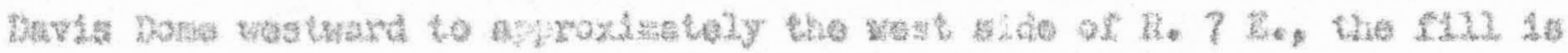

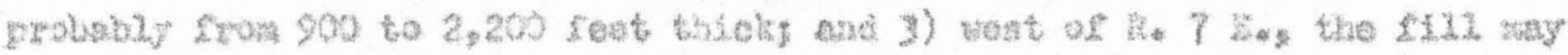

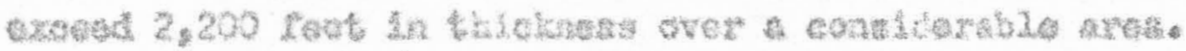




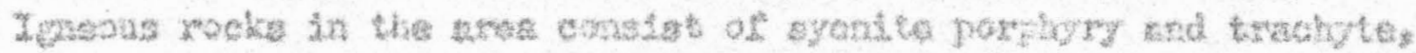

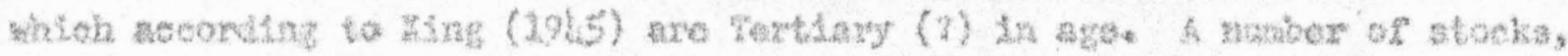

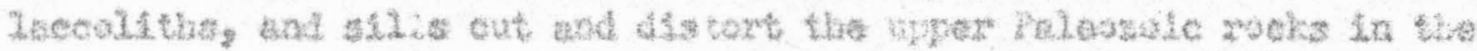

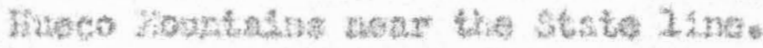

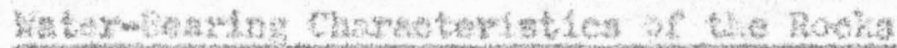

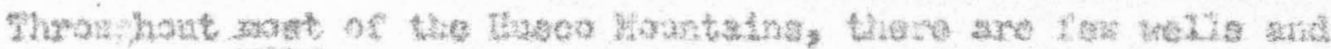

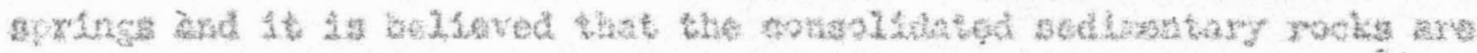

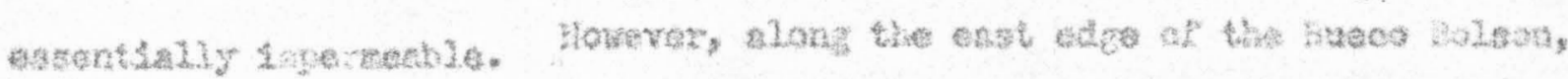

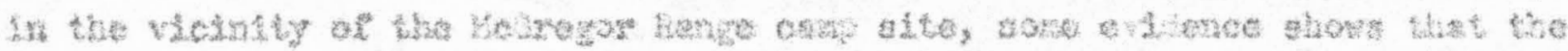

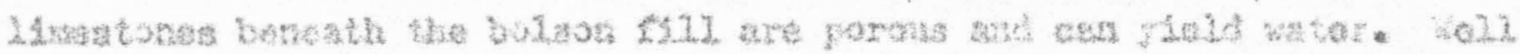

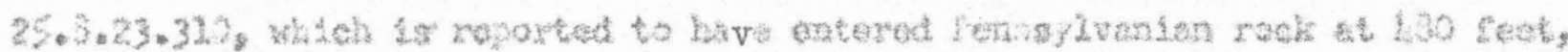

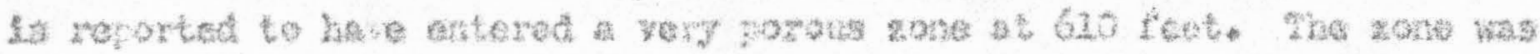

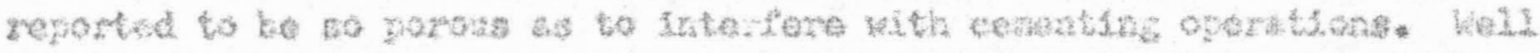

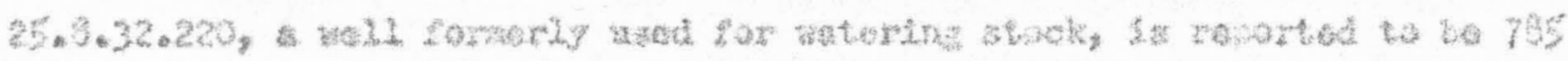

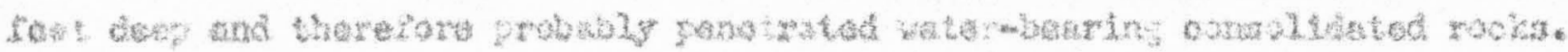

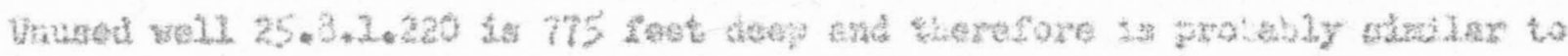

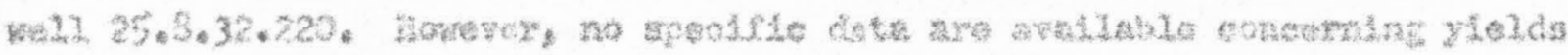
ag thesu was. 


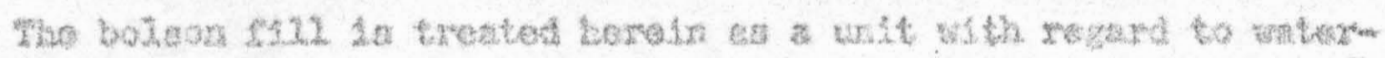

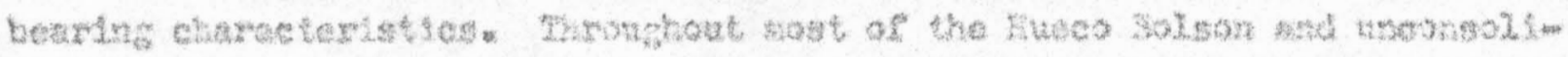

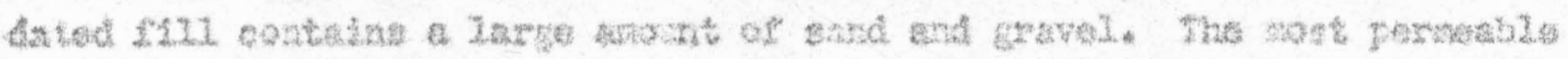

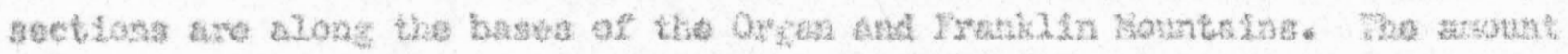

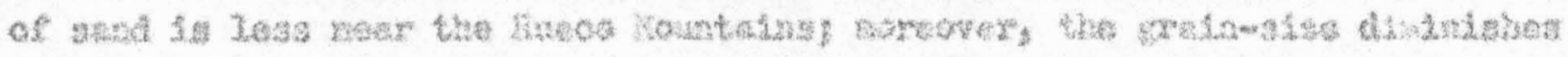

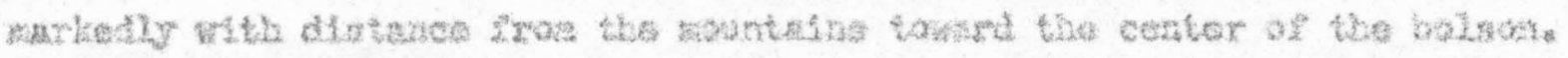

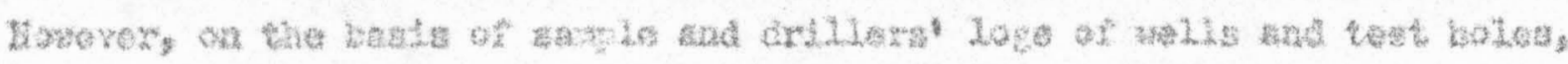

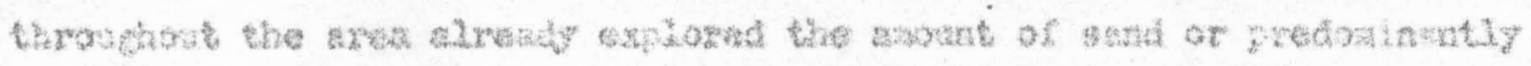

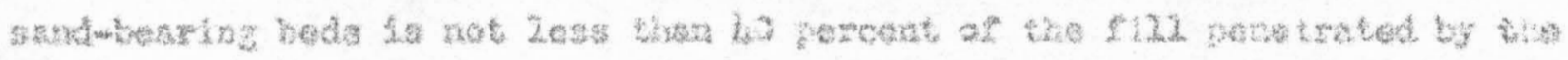

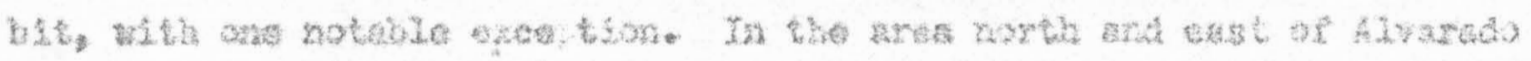

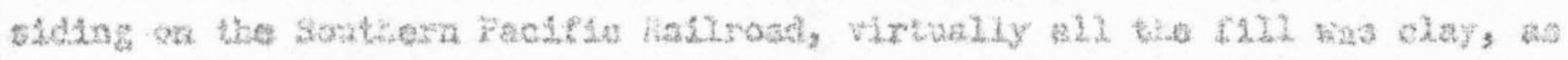

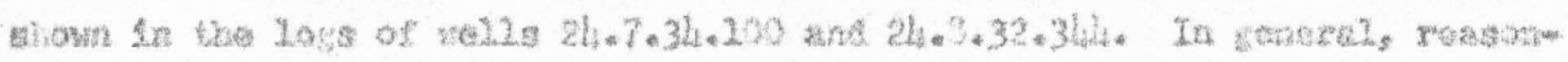
ab $3 y$ d.

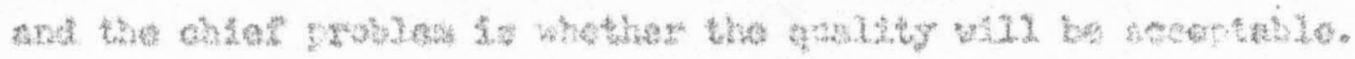

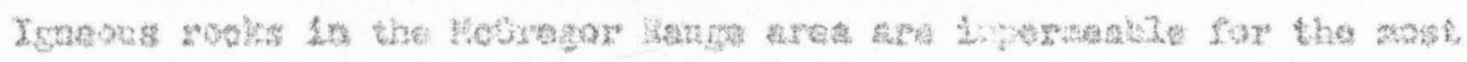

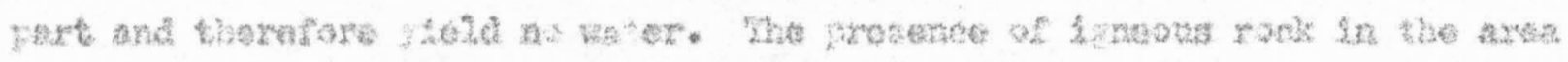

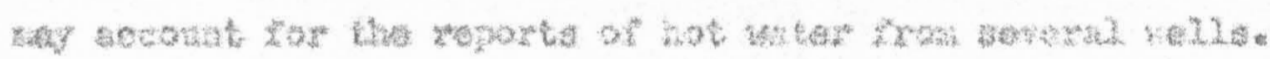




\section{Ditructiars}

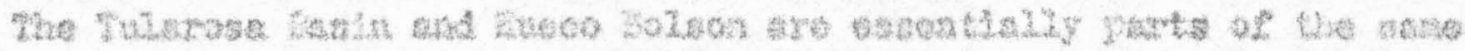

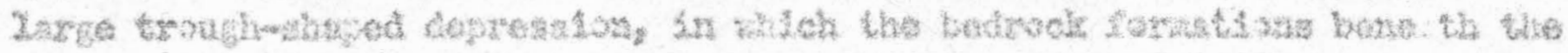

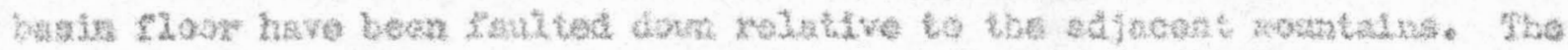

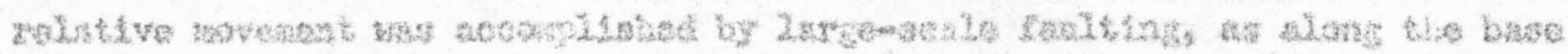

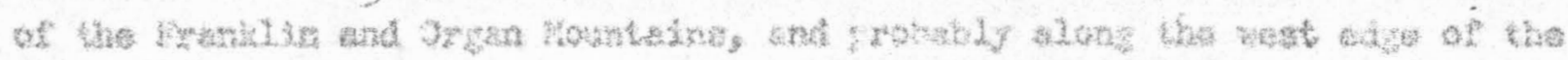

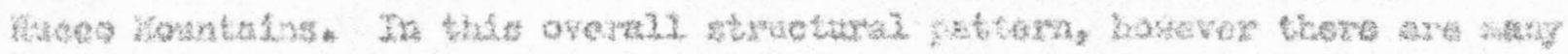

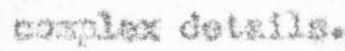

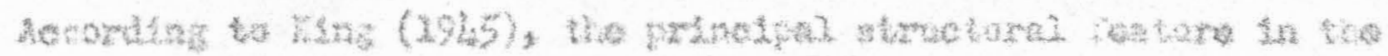

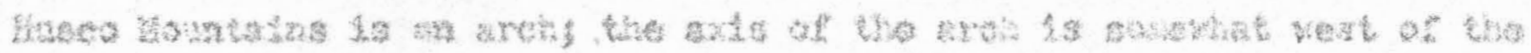

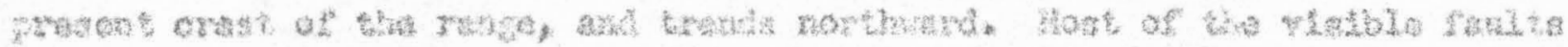

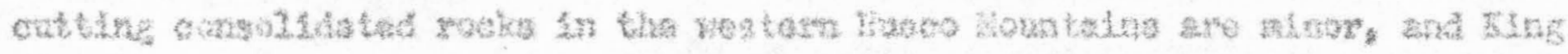

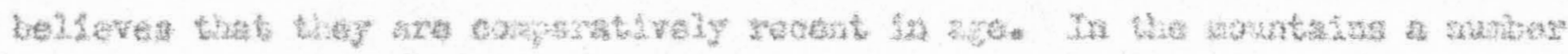

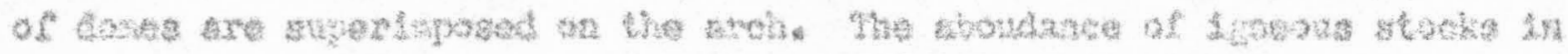

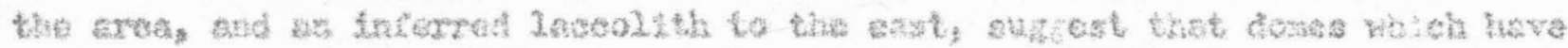

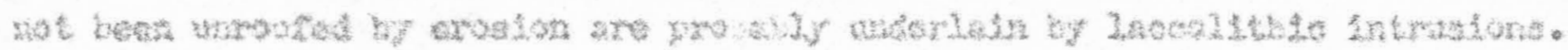

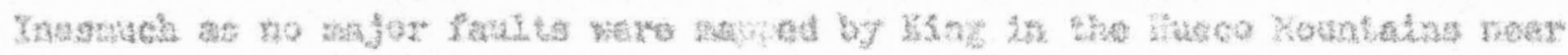

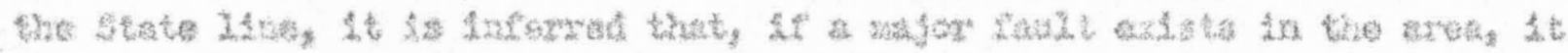
5.

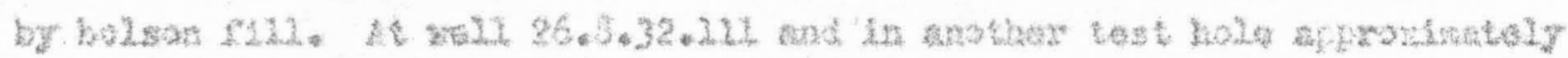

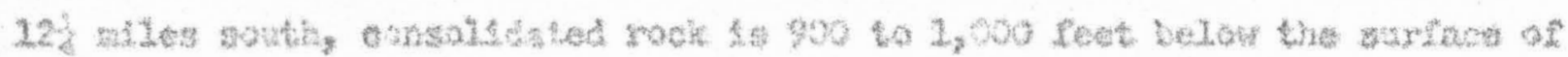

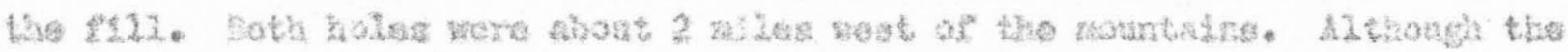

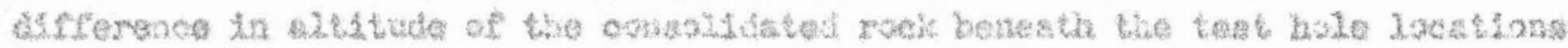

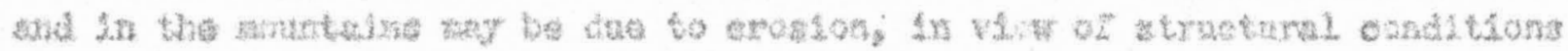

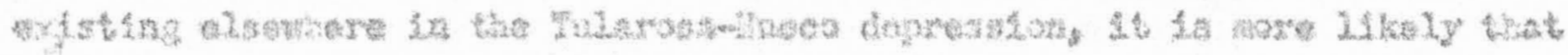

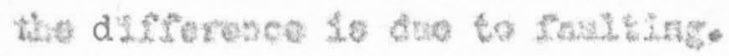




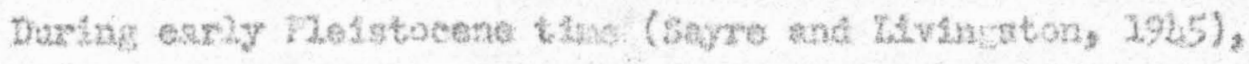

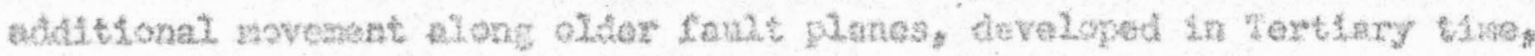

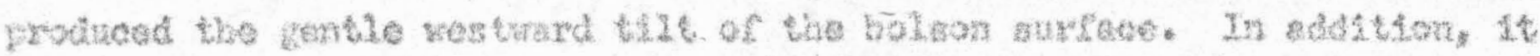

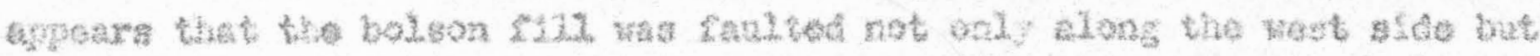

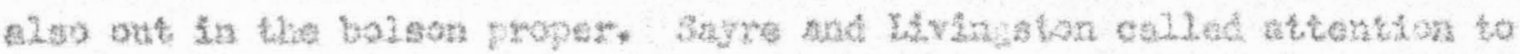

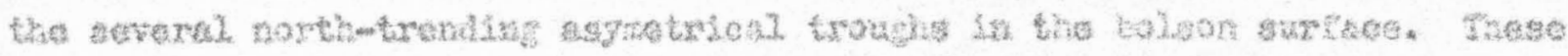

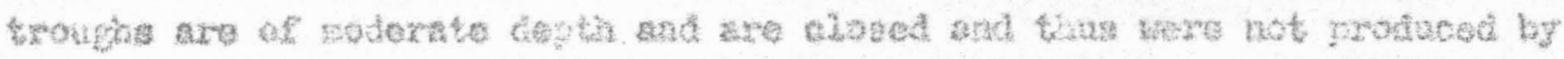

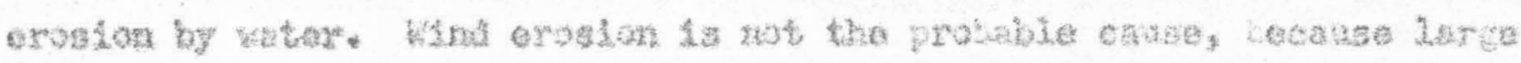

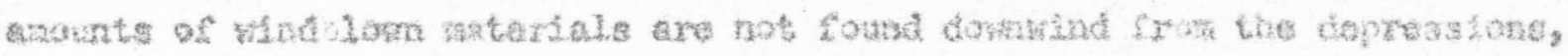

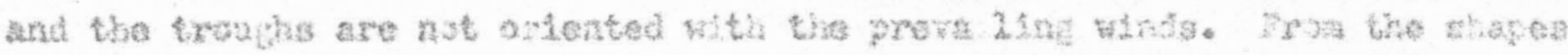

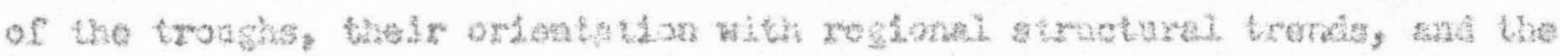

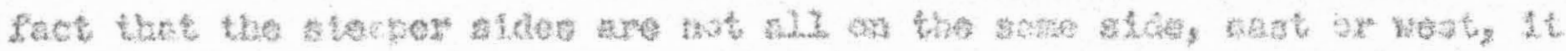

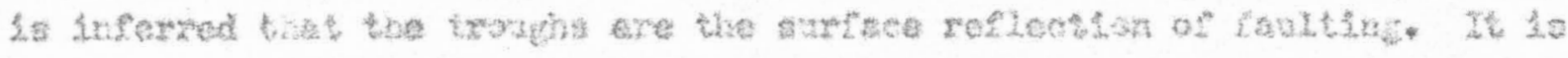

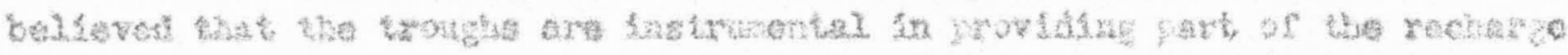

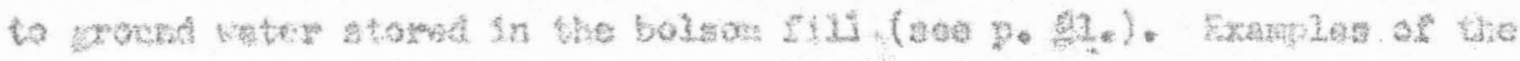

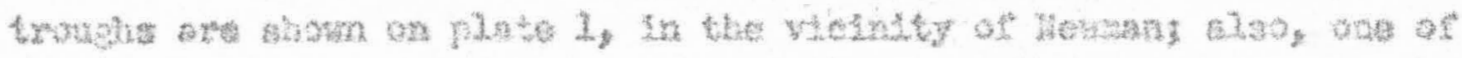

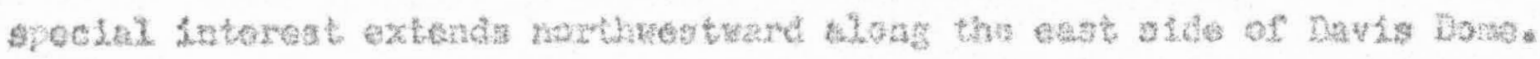

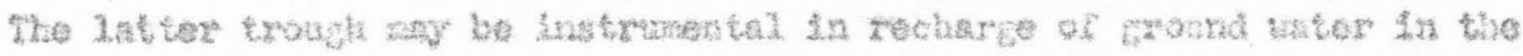

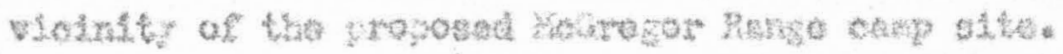




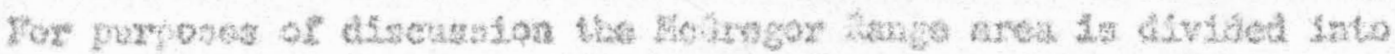

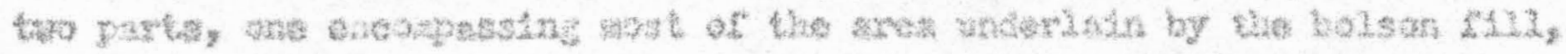

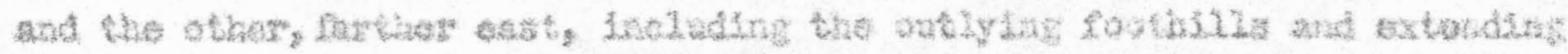

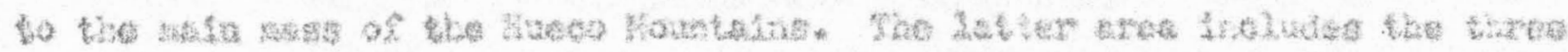

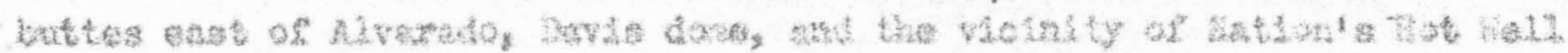
$(26.73, \cdot 3272)$

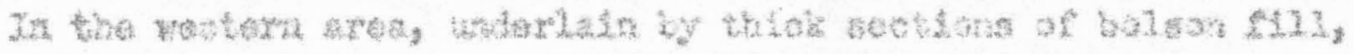

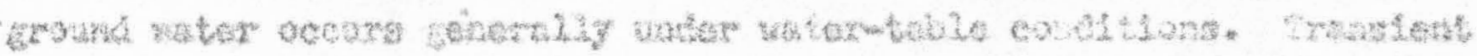

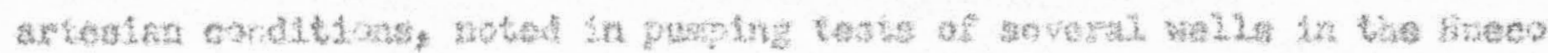

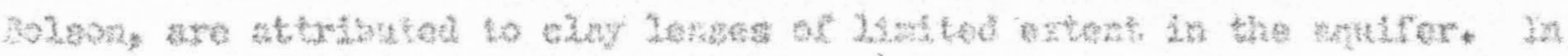

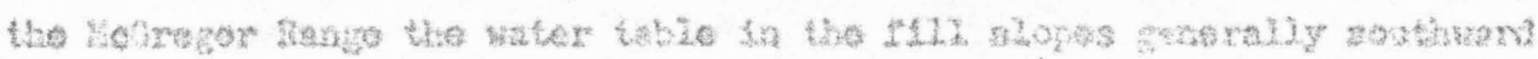

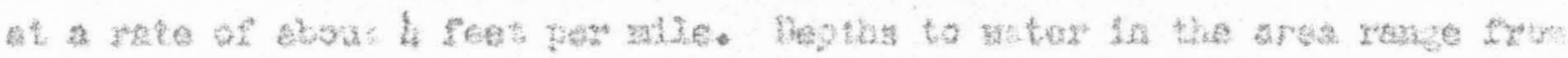

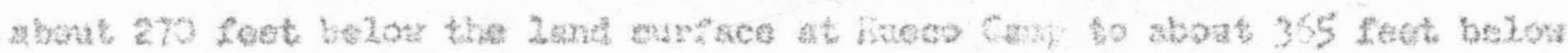

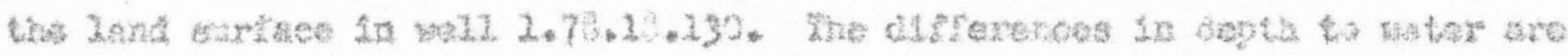

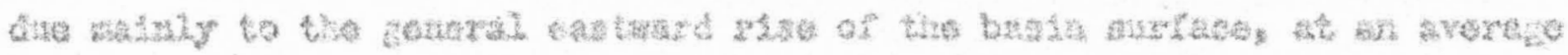

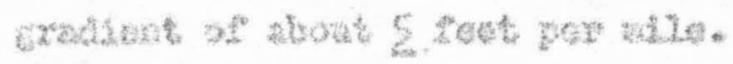




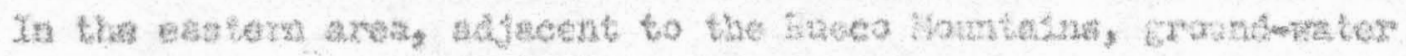

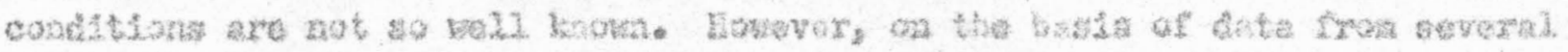

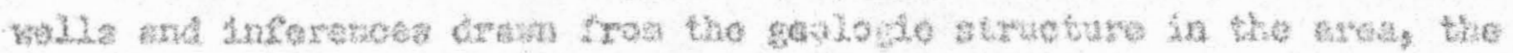

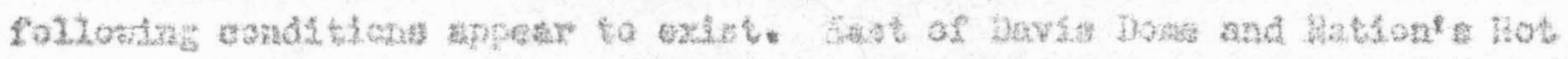

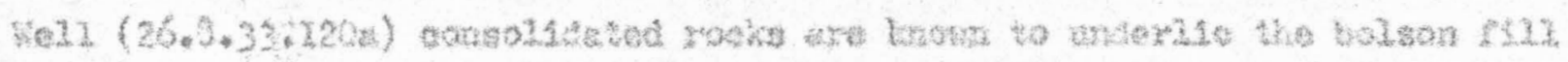

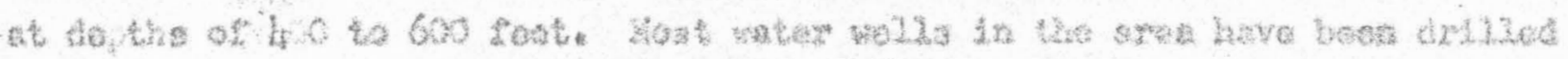

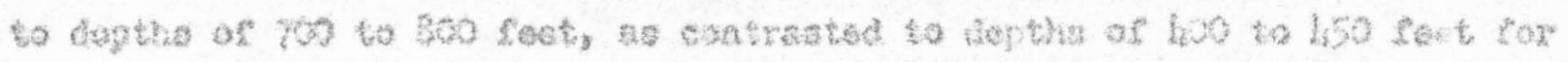

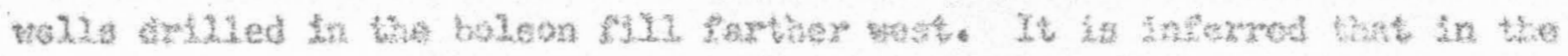

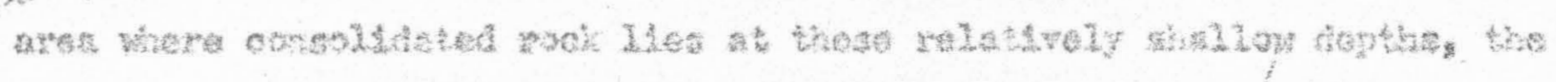

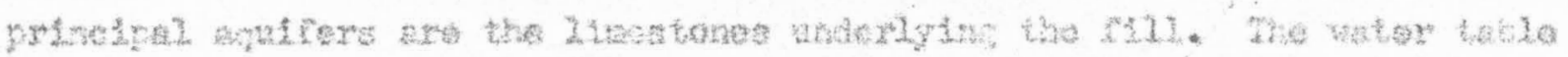

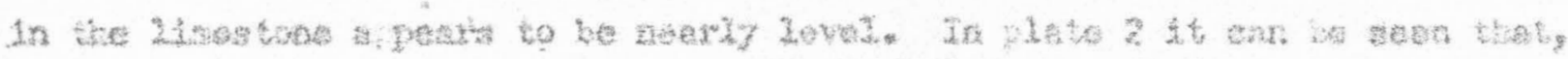

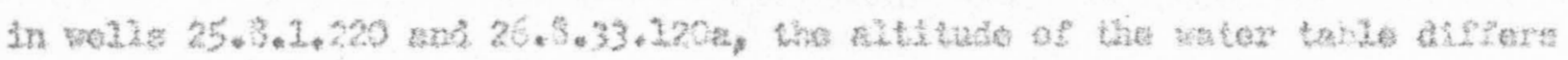

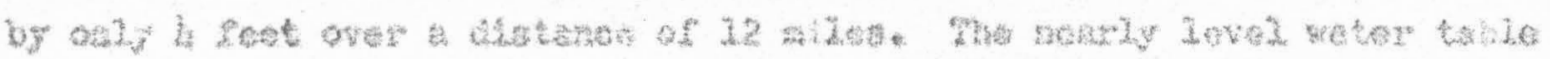

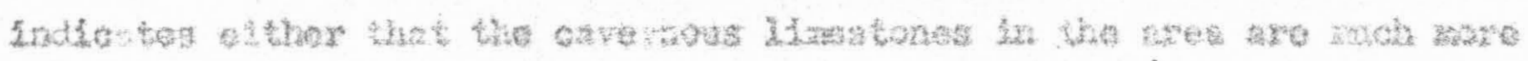

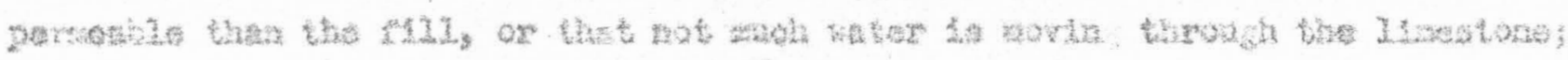

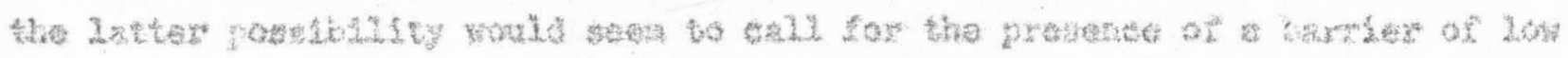

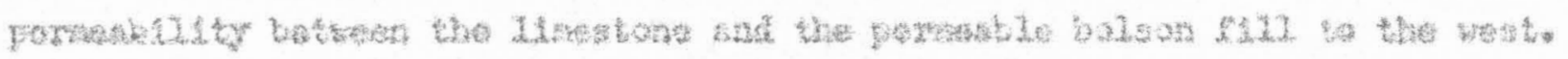

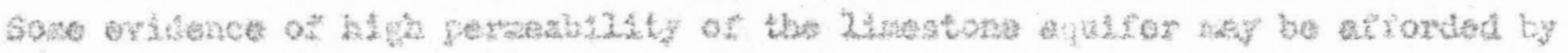

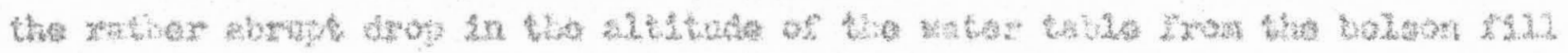

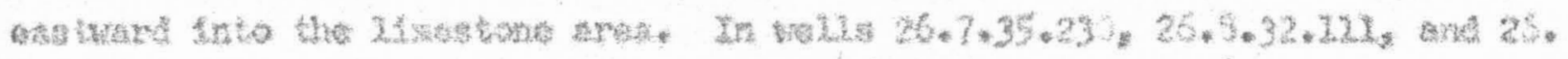

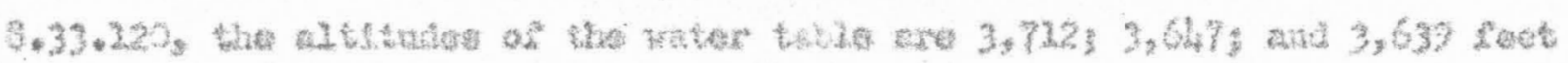

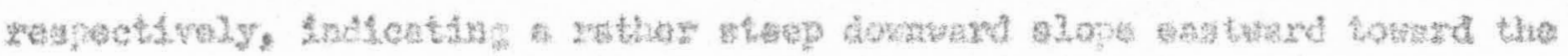

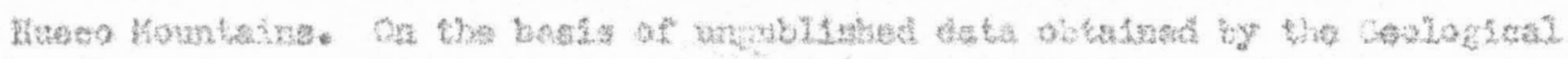

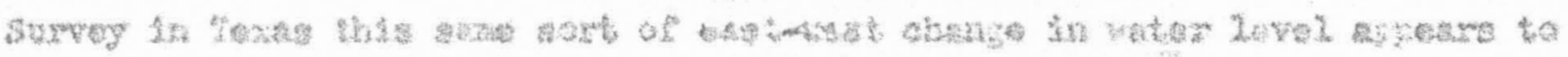

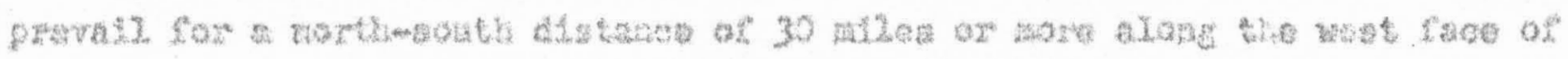




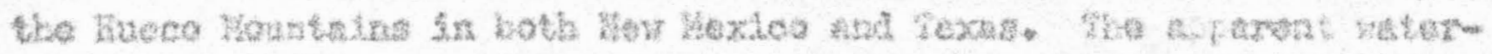

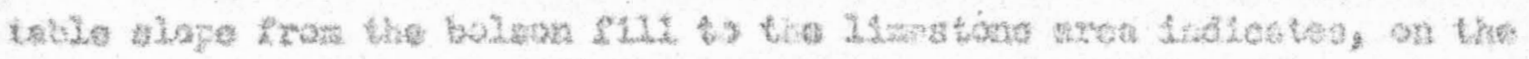

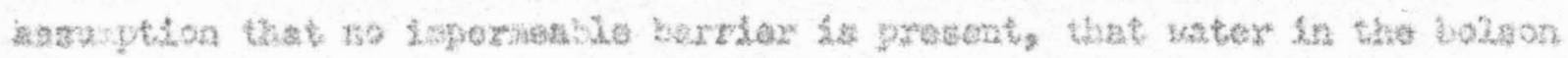

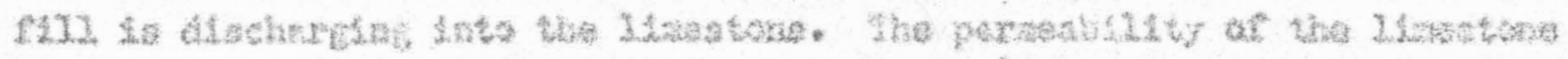

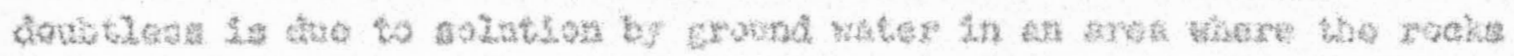

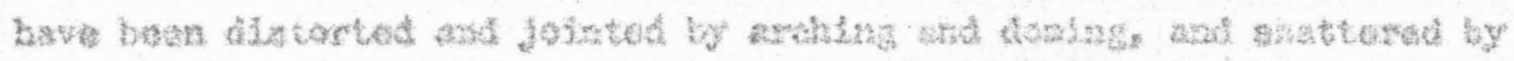

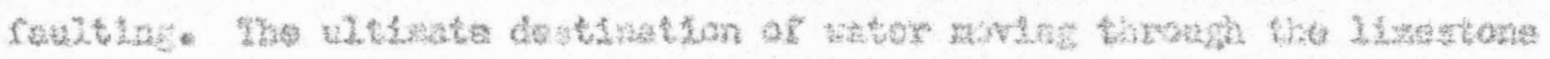

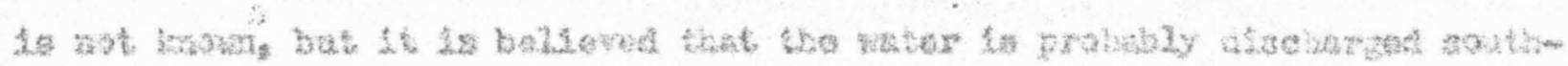

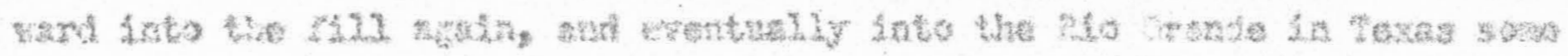

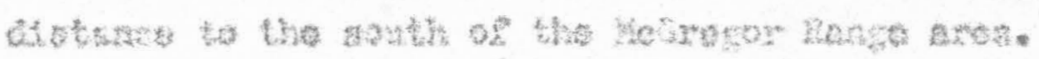

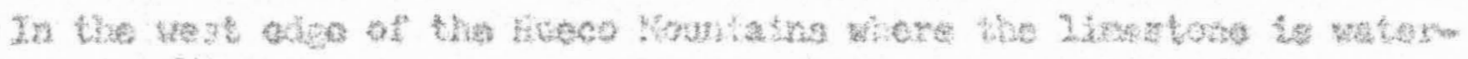

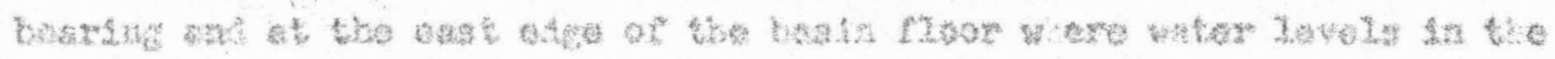

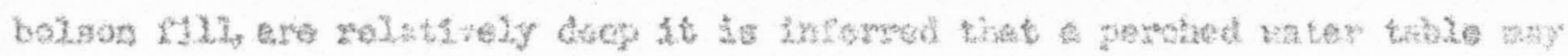

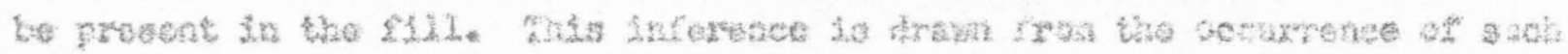

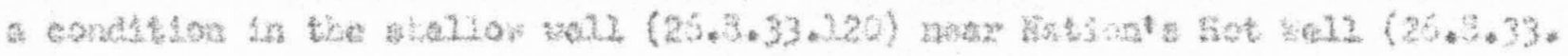

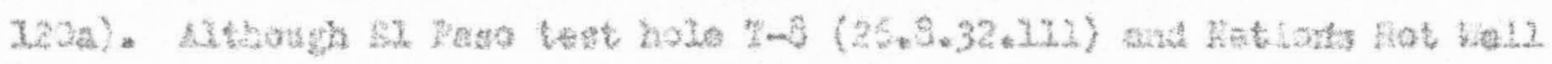

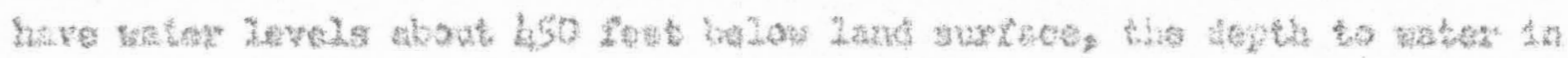

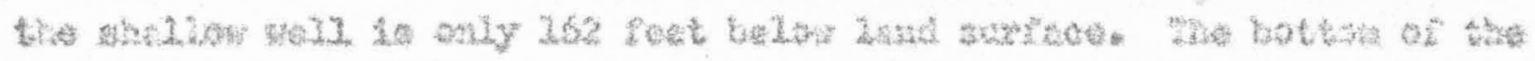

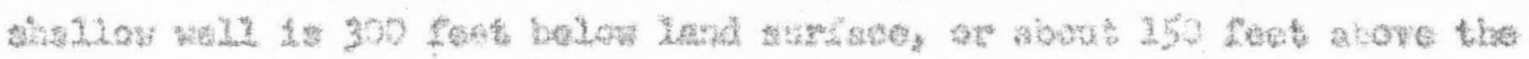

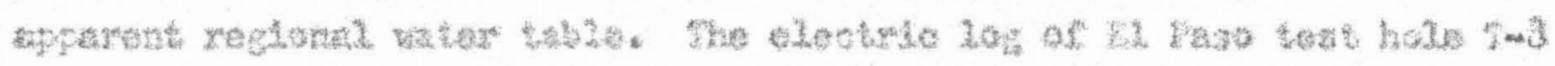

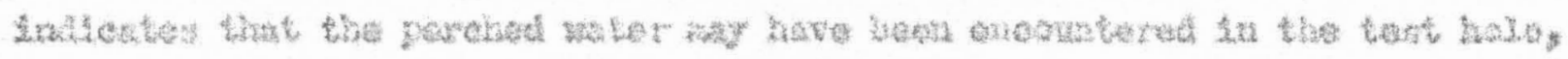

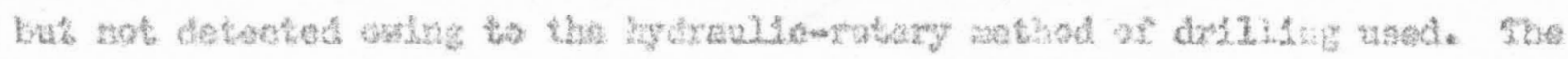

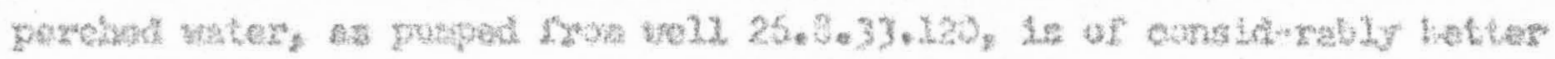

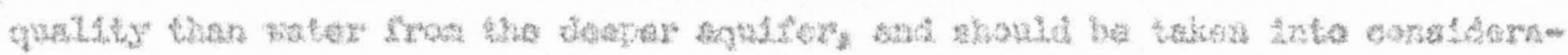

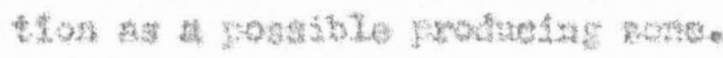




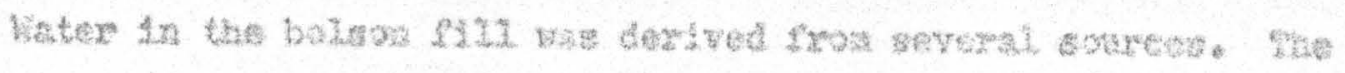

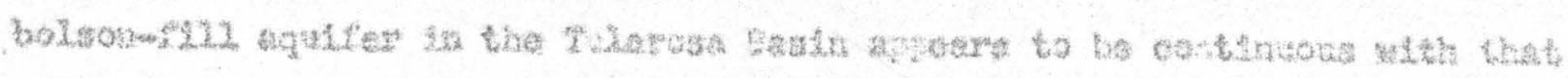

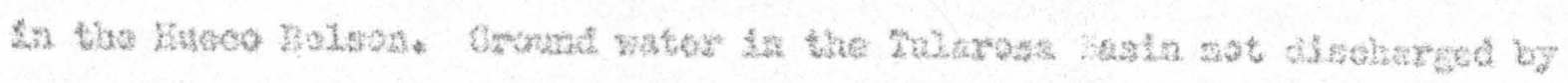

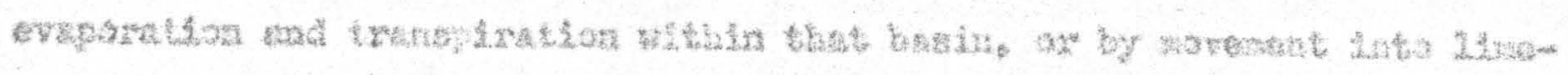

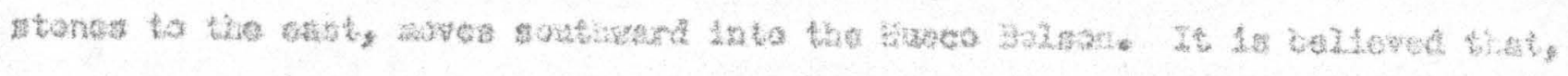

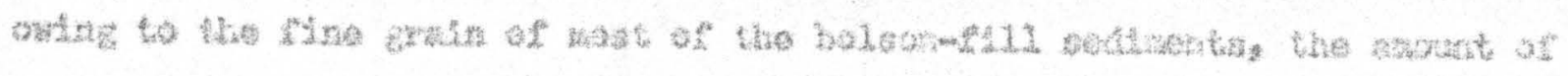

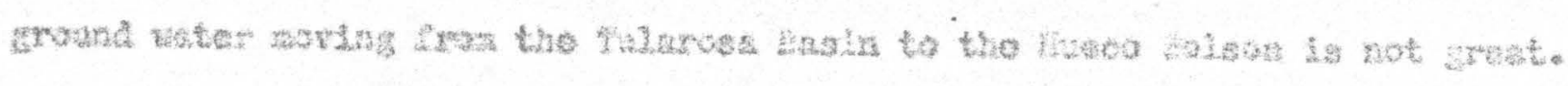

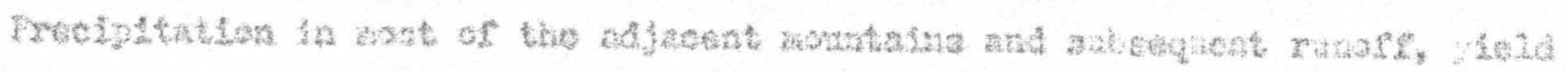

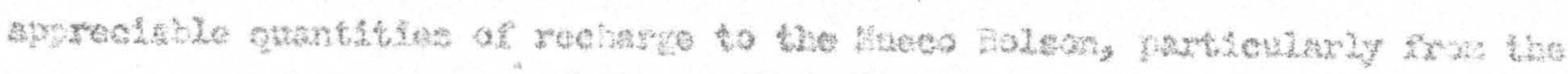

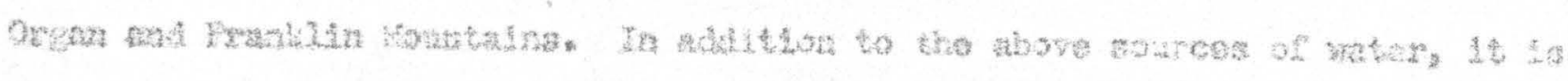

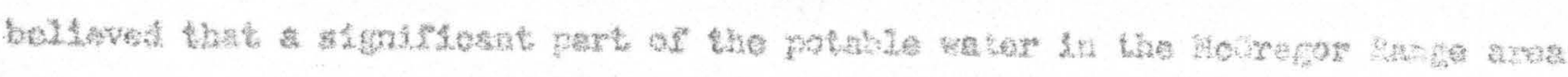

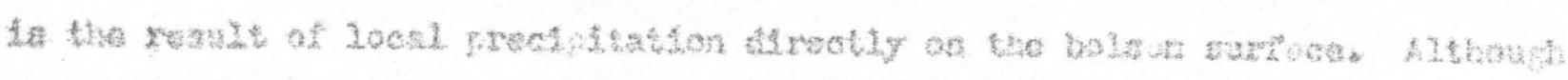

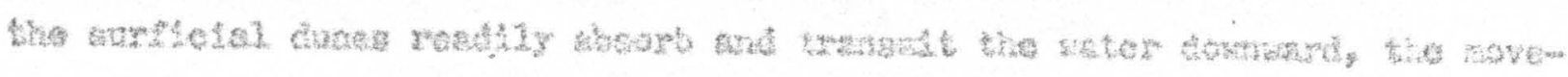

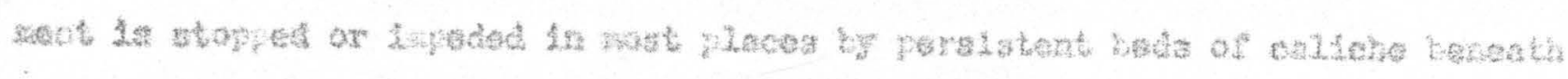

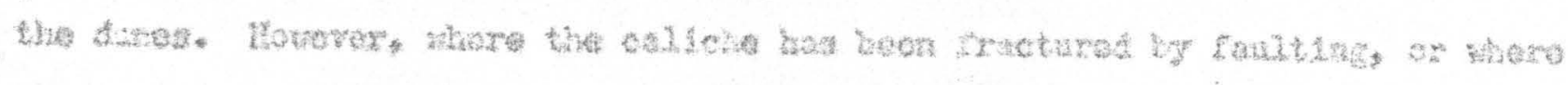

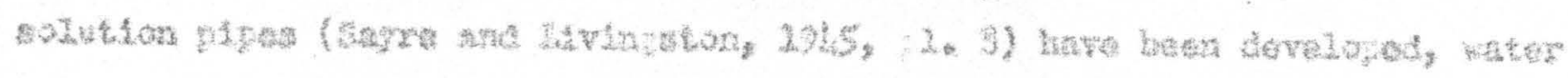

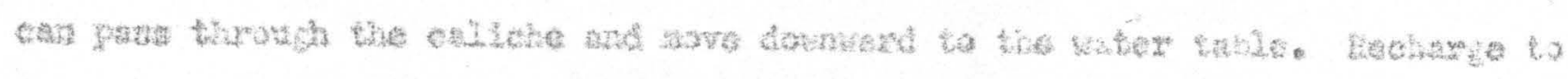

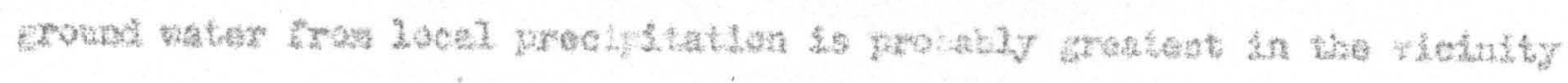

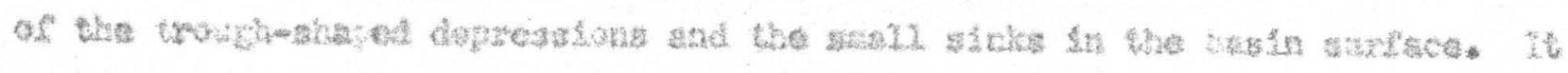

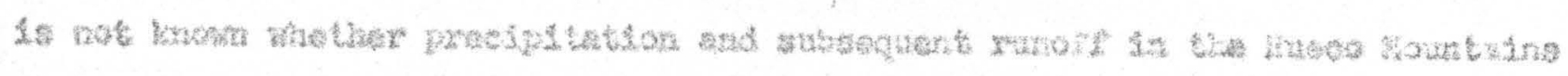

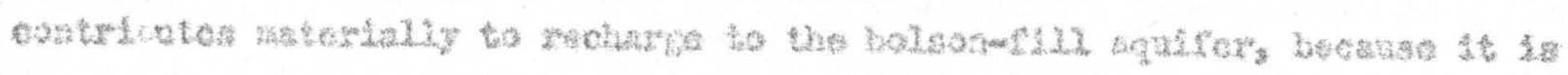

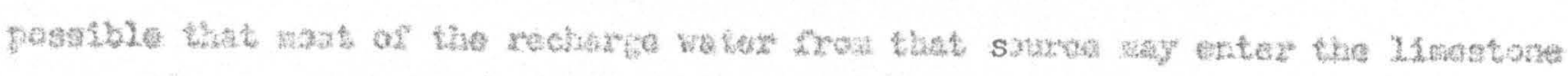

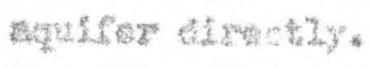

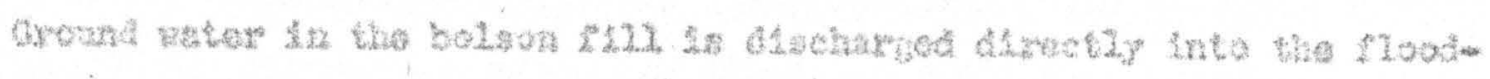

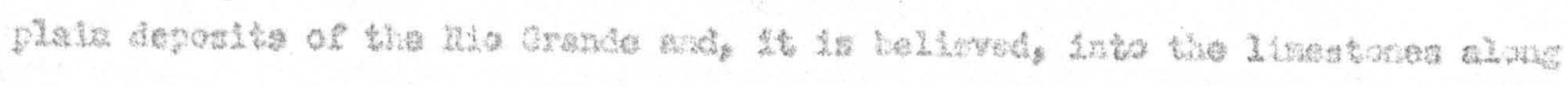

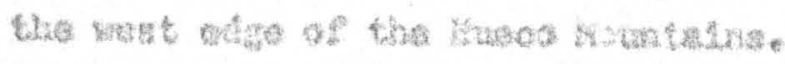




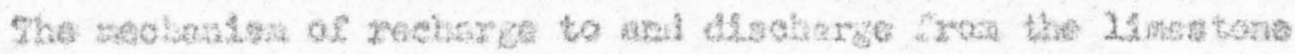

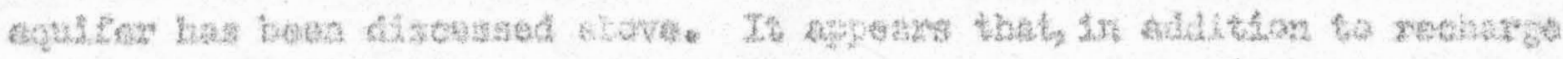

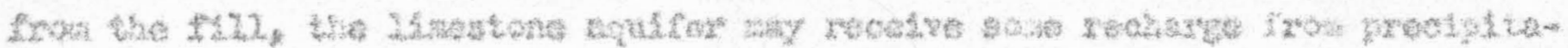

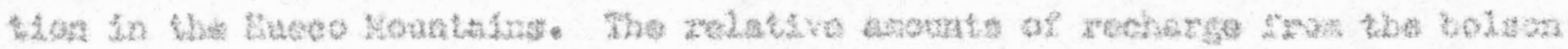

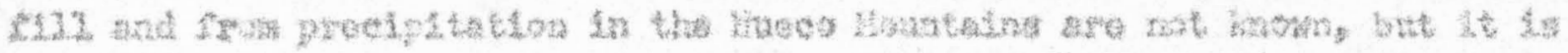

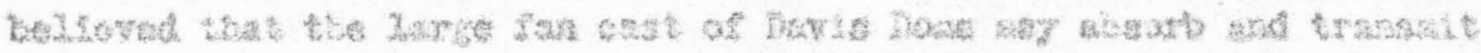

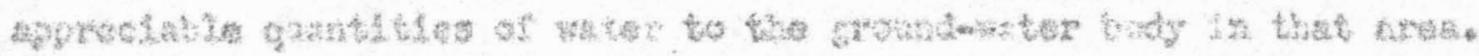




\section{Avglisbintt of oround later}

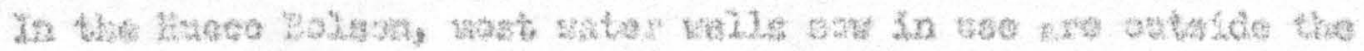

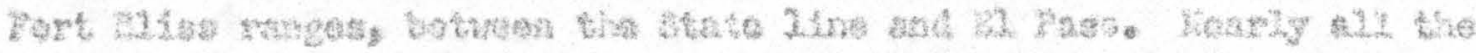

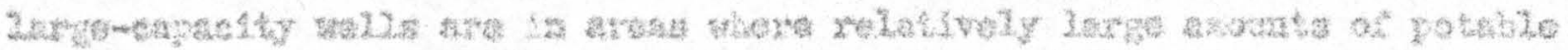

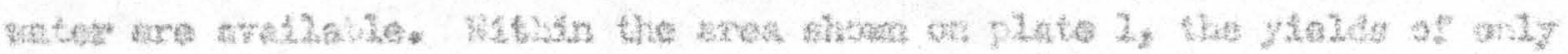

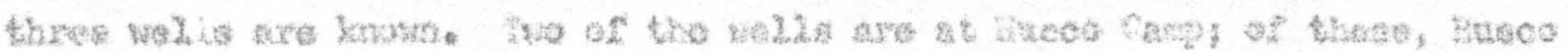

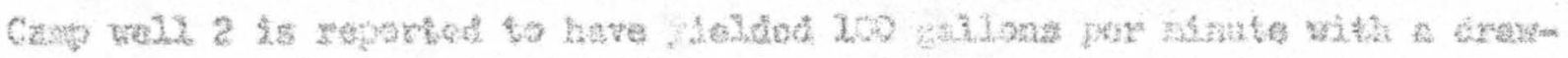

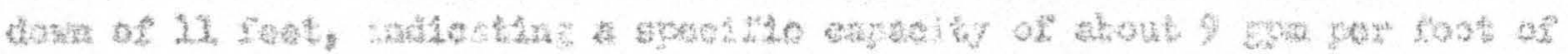

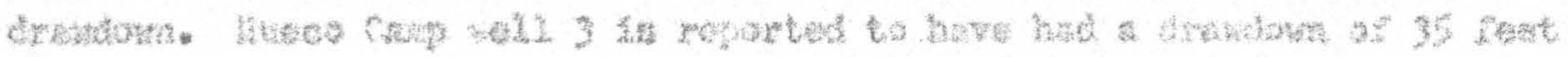

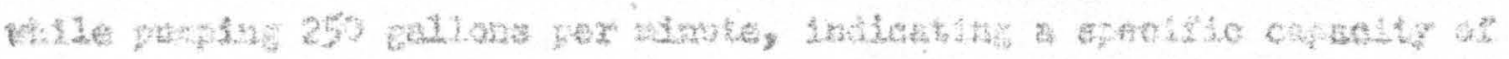

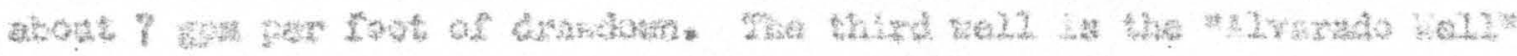

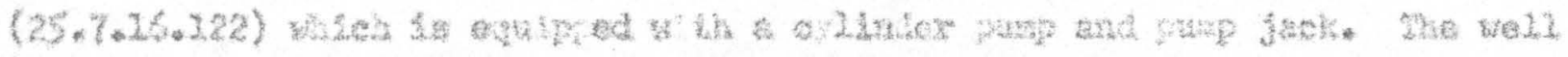

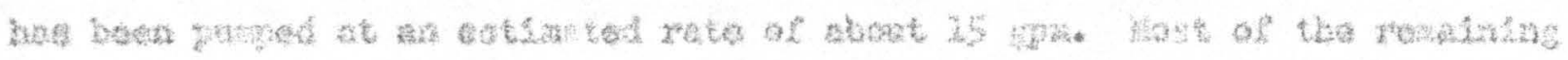

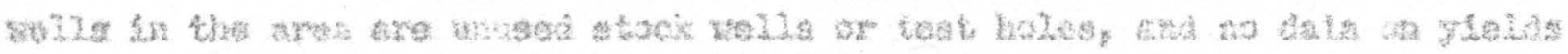

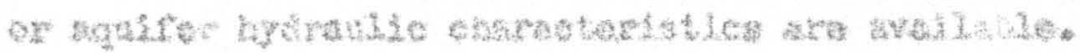

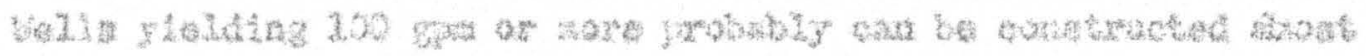

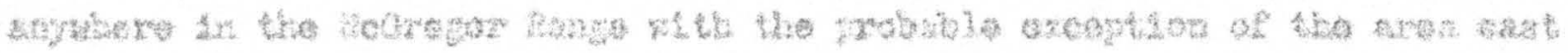

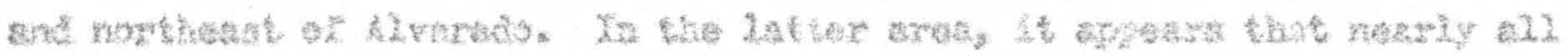

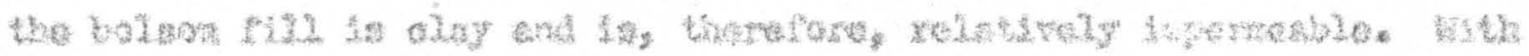

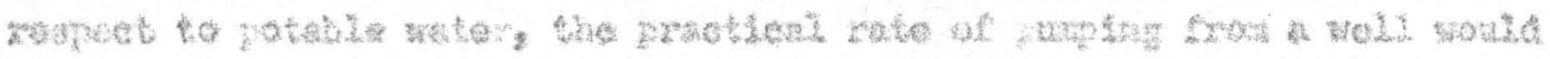

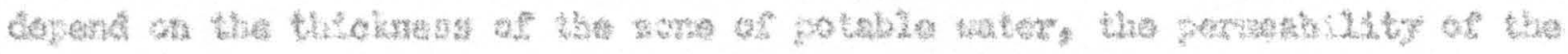

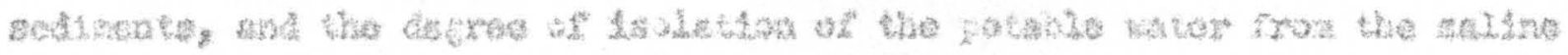

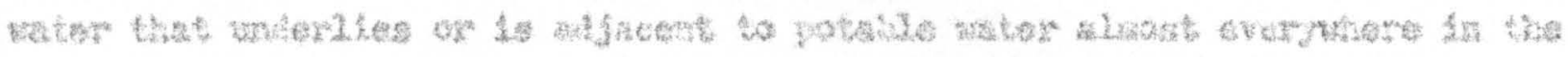
b.2. 


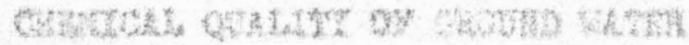

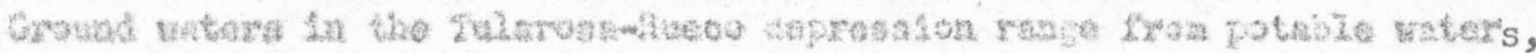

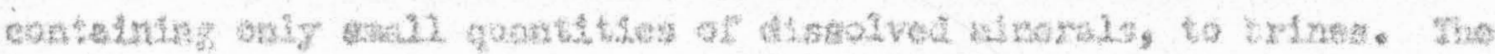

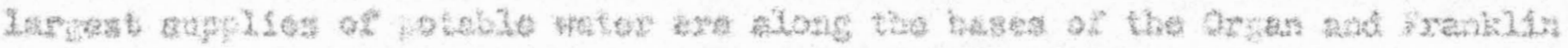

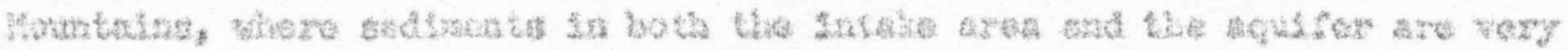

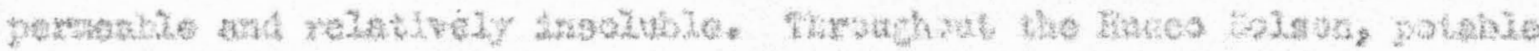

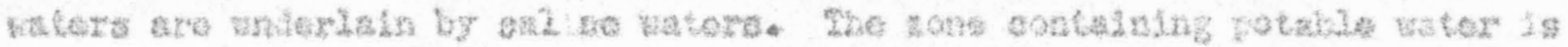

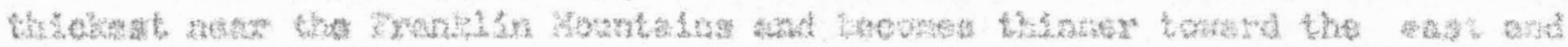

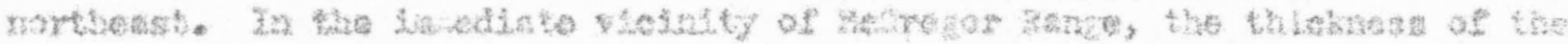

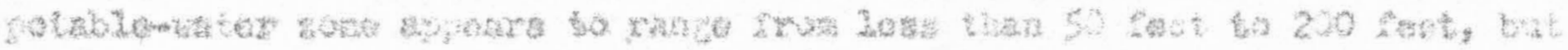

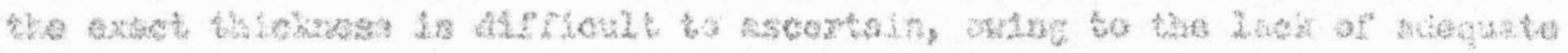

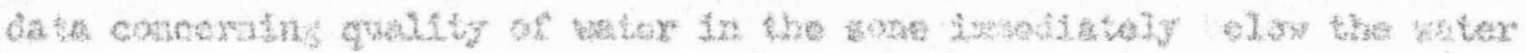

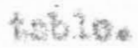

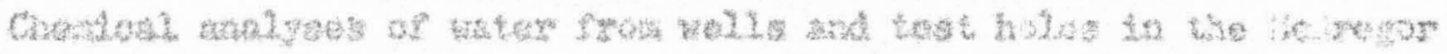

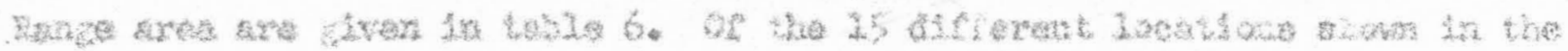

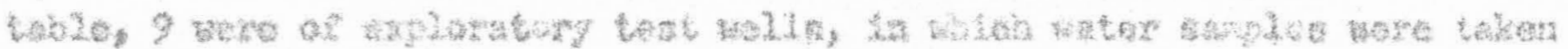

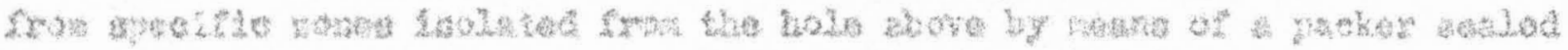
Afals

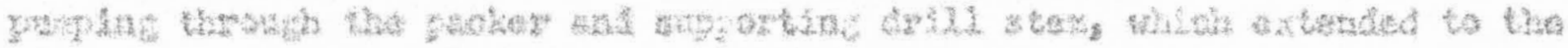

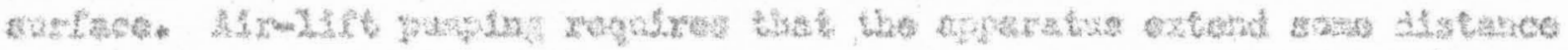

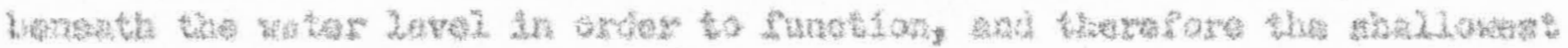

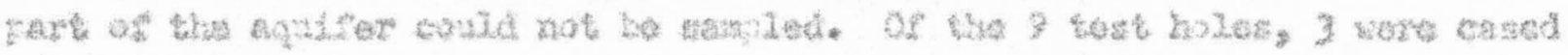
to bo wath

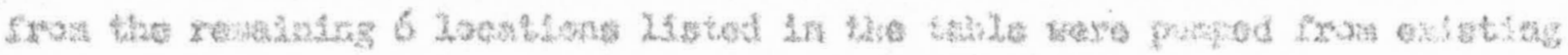
volite. 


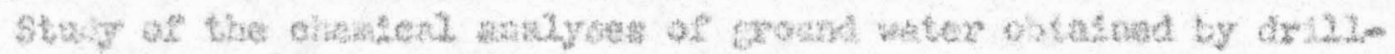

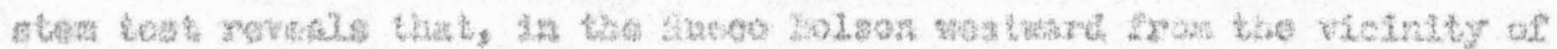

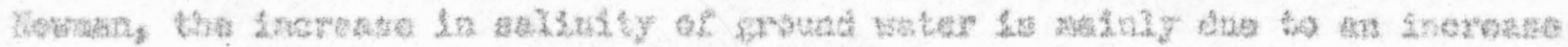

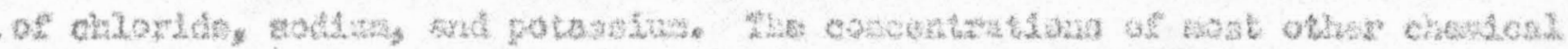

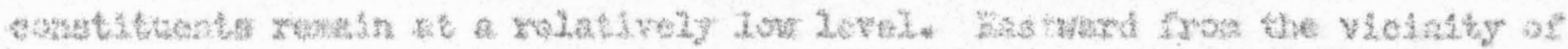

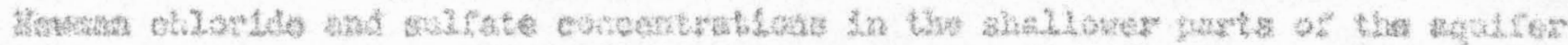

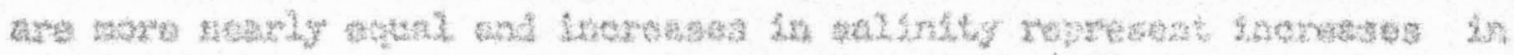

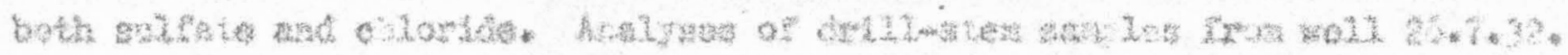

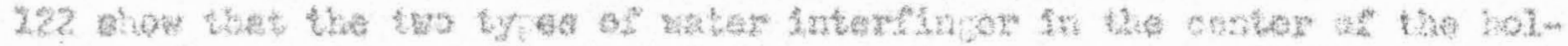

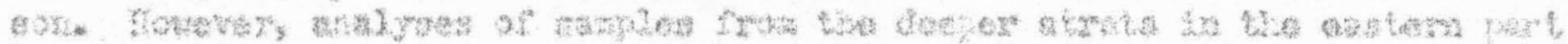

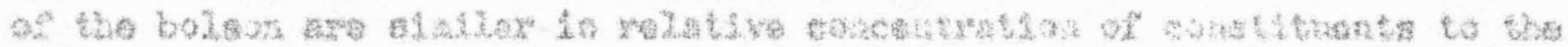

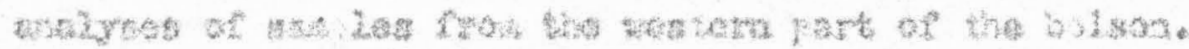

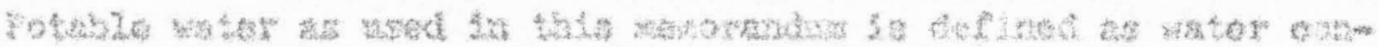

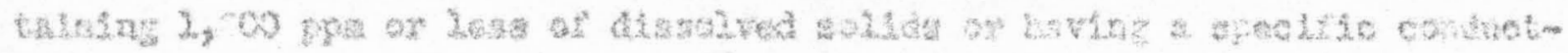

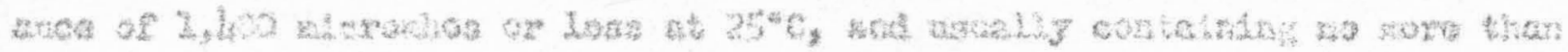

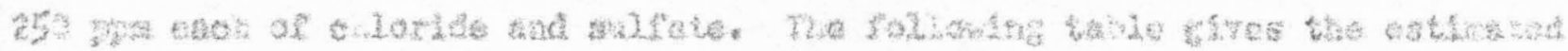

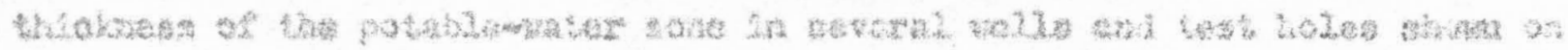

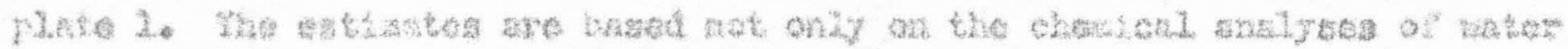

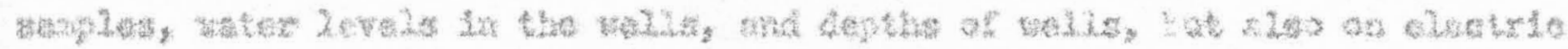

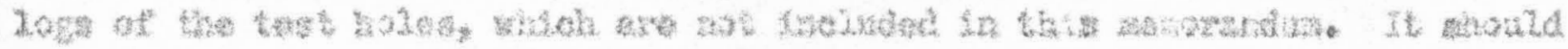

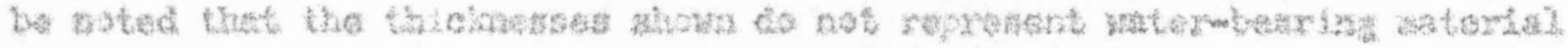

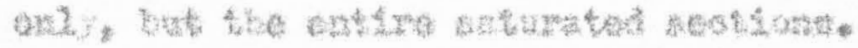


Thb? 2, patrblewater rons to

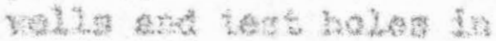

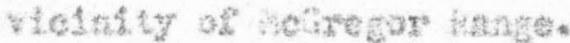

\begin{tabular}{|c|c|c|}
\hline $\begin{array}{l}\text { Wel1 } \\
\text { number }\end{array}$ & $\begin{array}{c}\text { Known thickness } \\
\text { (feet) }\end{array}$ & $\begin{array}{l}\text { Probable maximum } \\
\text { thickness (feet) }\end{array}$ \\
\hline $25.5 .20 \cdot 3340$ & $*$ & 200 \\
\hline $25 \cdot 6 \cdot 34 \cdot 21$ & $\because$ & 70 \\
\hline $25.7 .26 \cdot 222$ & - & 73 \\
\hline $23 \cdot 5 \cdot 34,13 \%$ & 13 & H* \\
\hline $26.7 \cdot 32 \cdot 122$. & - & 19 \\
\hline 25.7 .35 .230 & 35 & 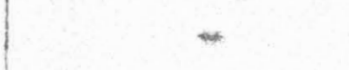 \\
\hline $25+3+32+1 x 1$ & $\infty$ & 30 \\
\hline $26.3 .33 \cdot .29$ & 2372 & - \\
\hline $3,4,2,131$ & 49 & 130 \\
\hline $2.85 \cdot 4 \cdot 222$ & $\ldots$ & 60 \\
\hline 2. $20.24 \cdot 333$ & 242 & - \\
\hline
\end{tabular}

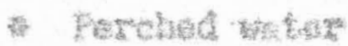




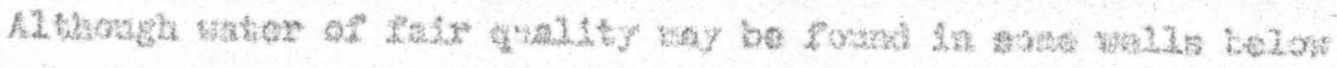

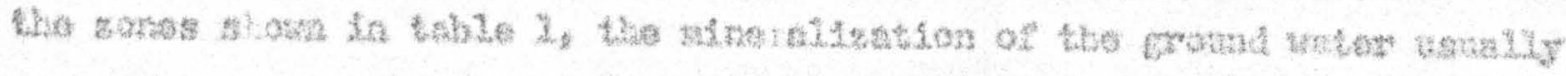

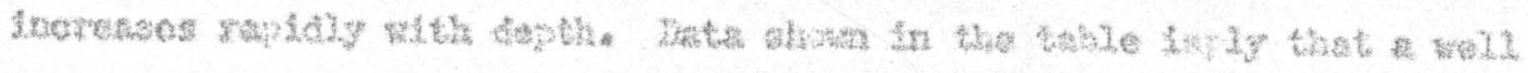

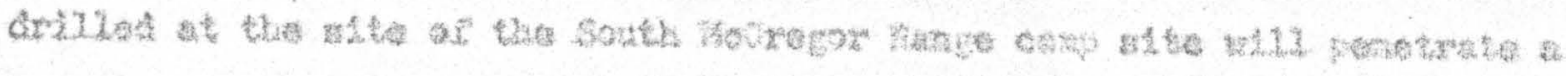

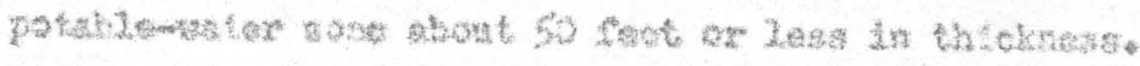

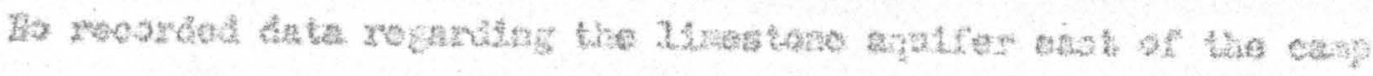

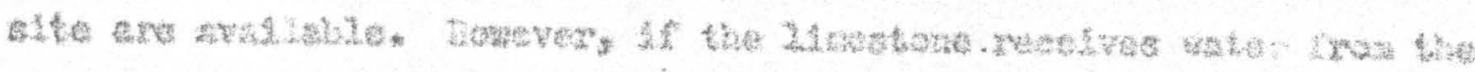

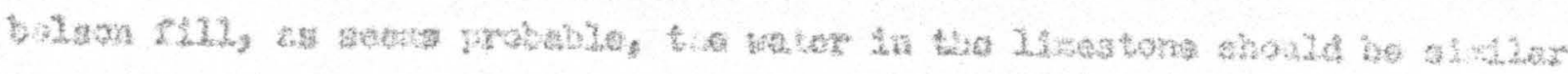

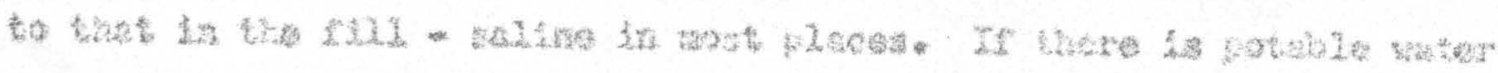

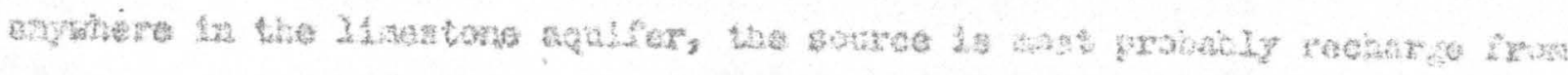

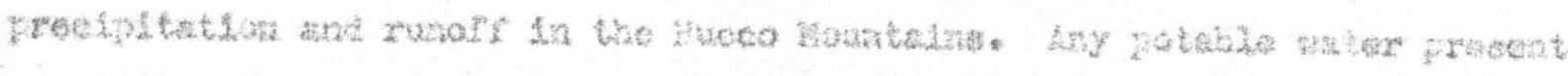

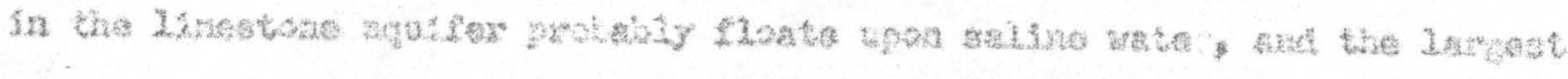

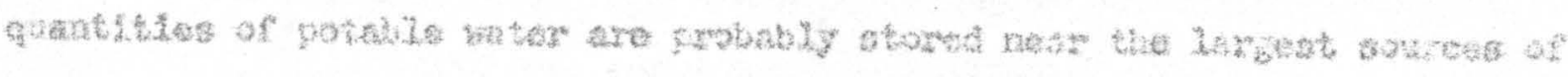

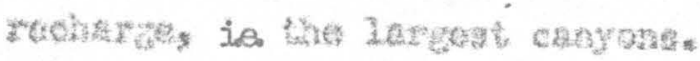




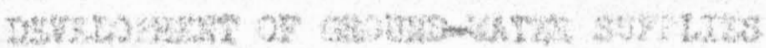

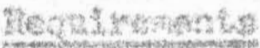

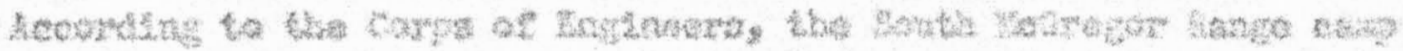

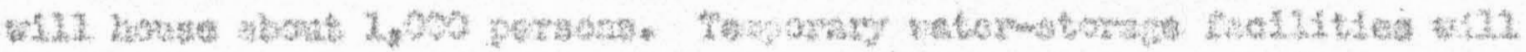

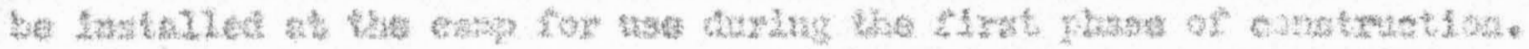

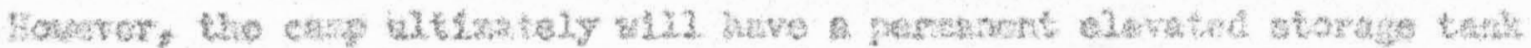

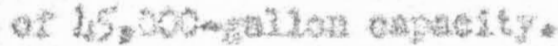

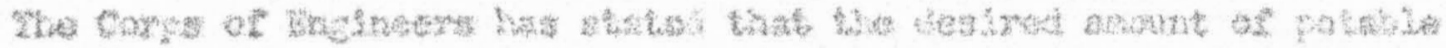

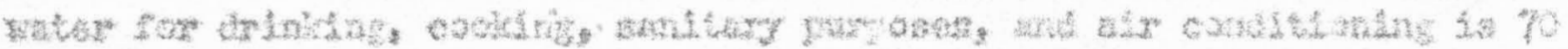

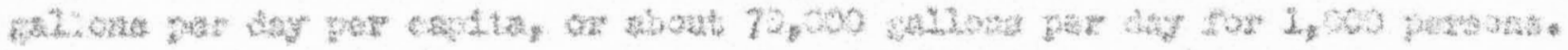

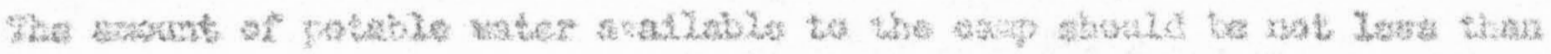

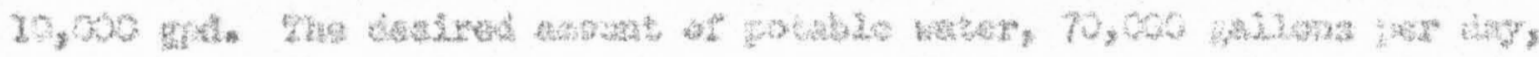

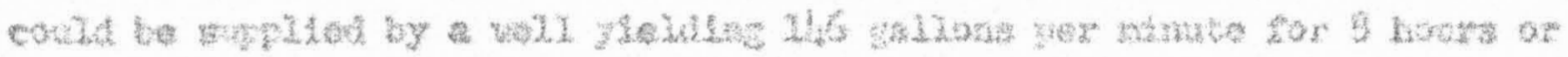

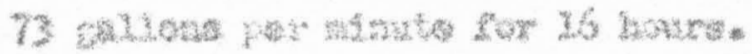

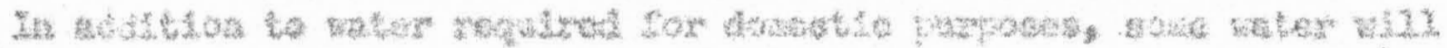

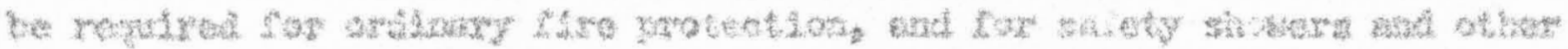

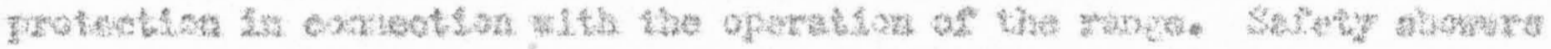

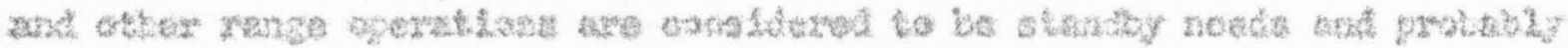

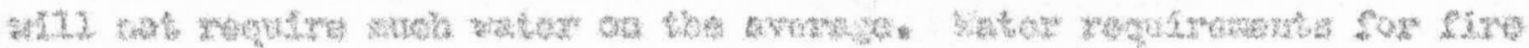

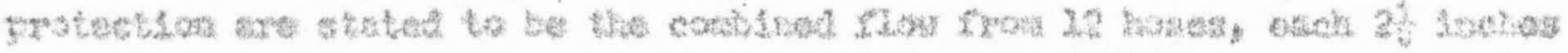

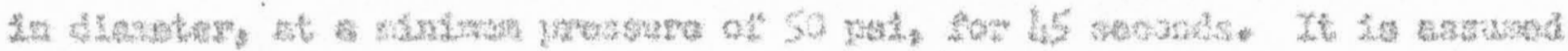

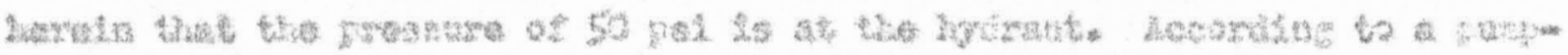

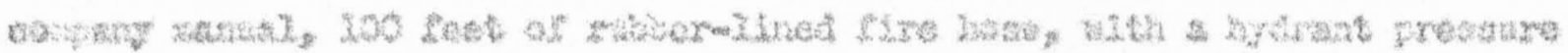

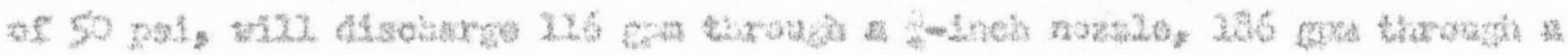

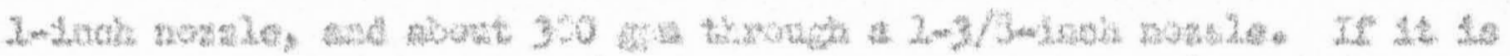

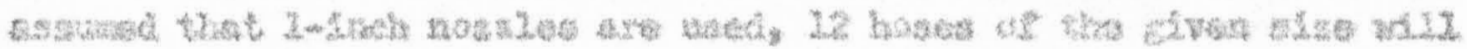




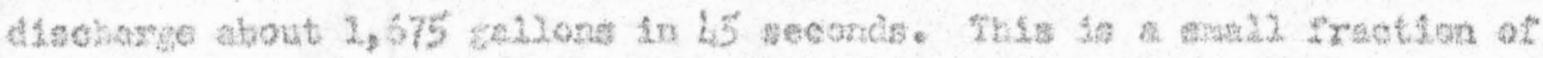

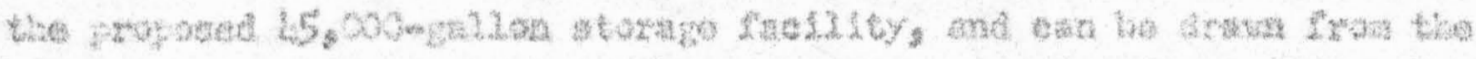

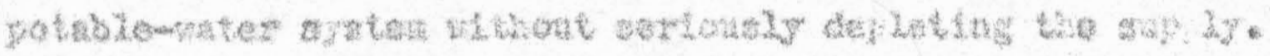

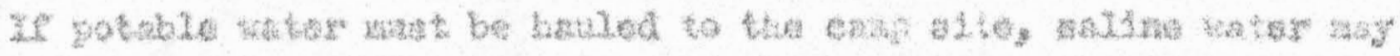

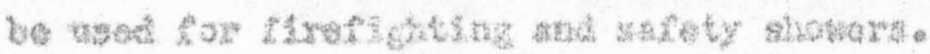

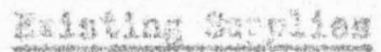

matro

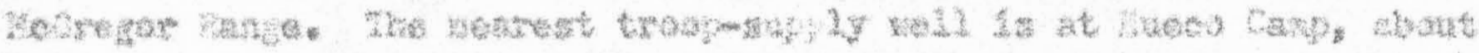

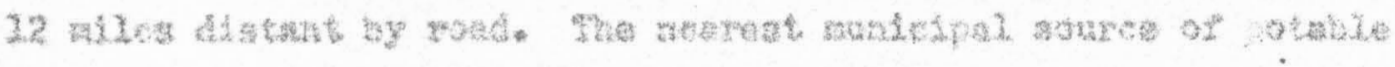

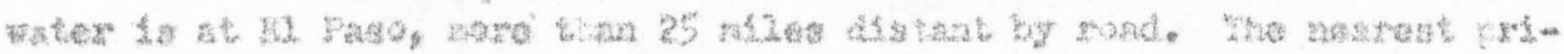

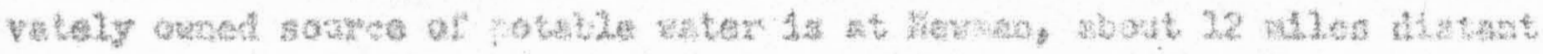
dey rosid. 


\section{Exploratory Draling in the frese}

On the brats of data given in preceding acturas the followng

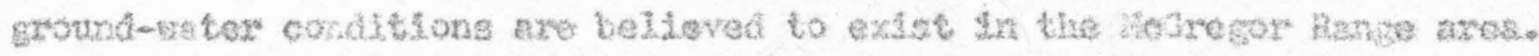

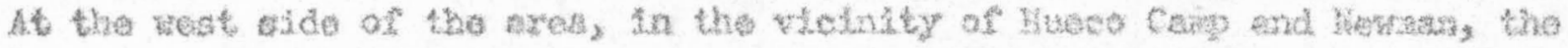

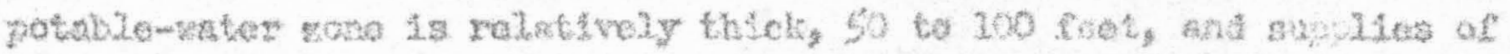

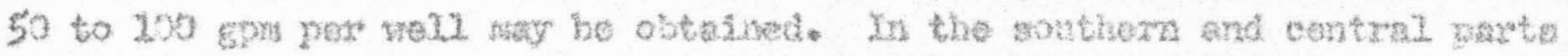

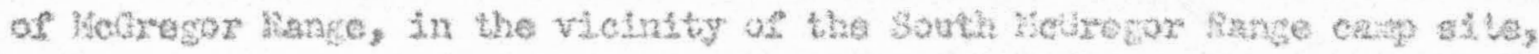

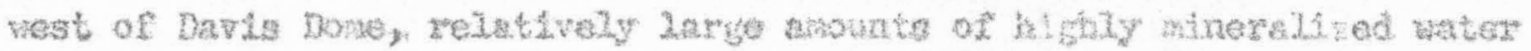

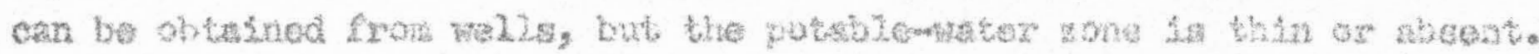

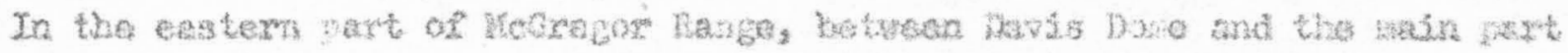

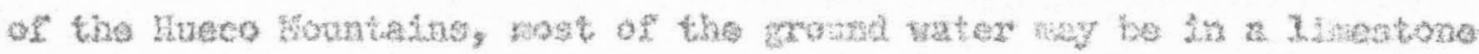

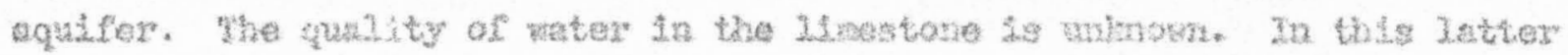

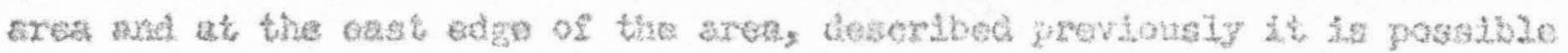

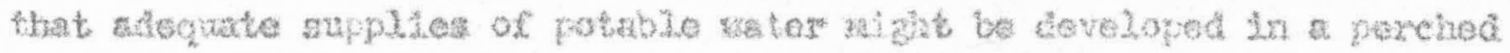

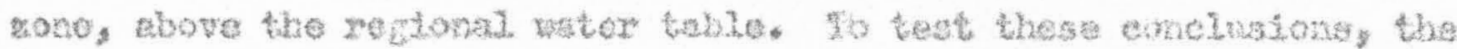

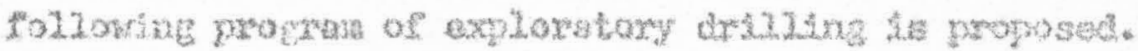




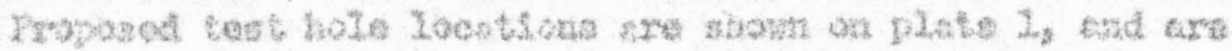

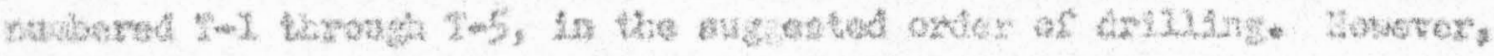

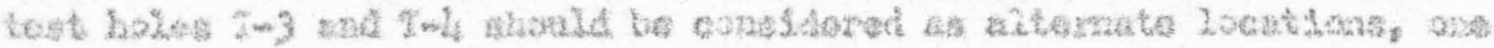

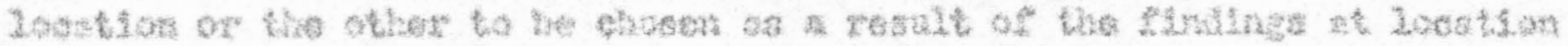

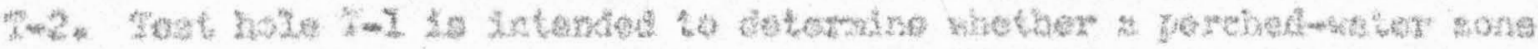

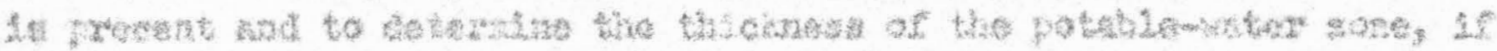

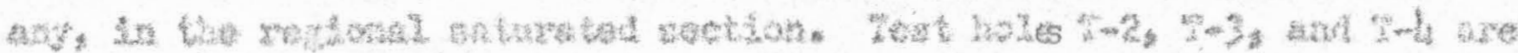

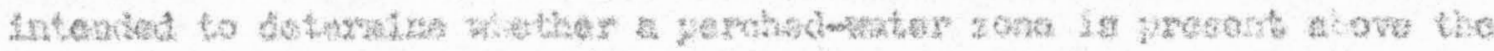

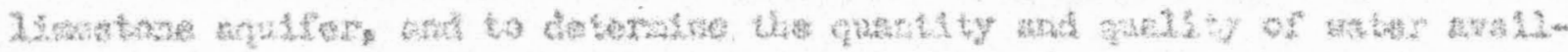

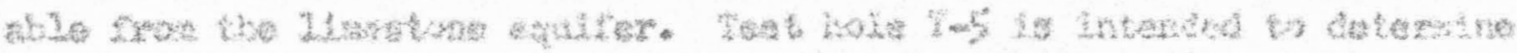

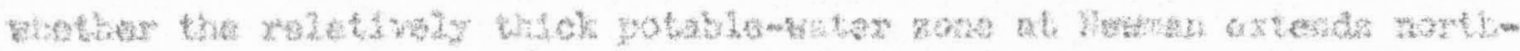

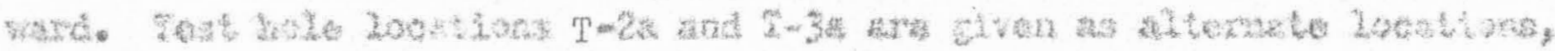

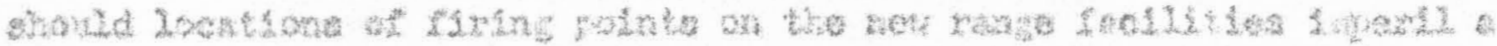

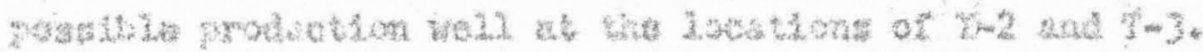

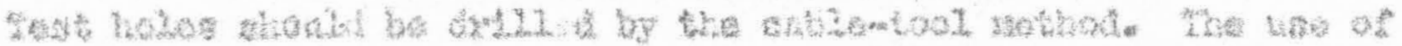

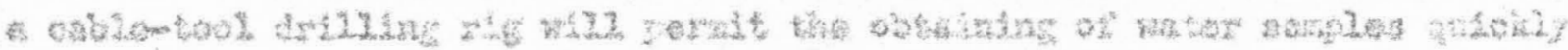

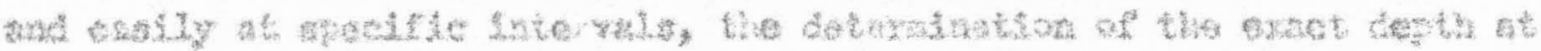

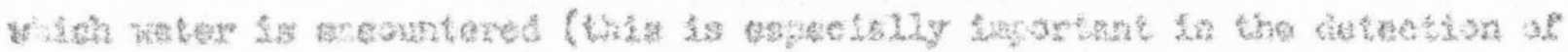

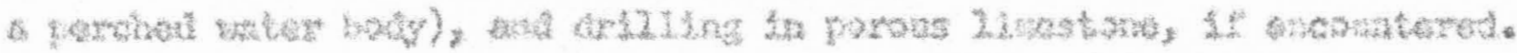

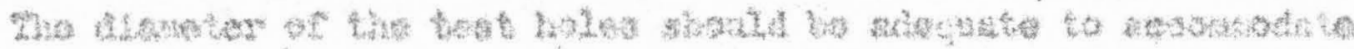

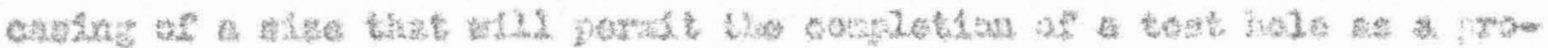

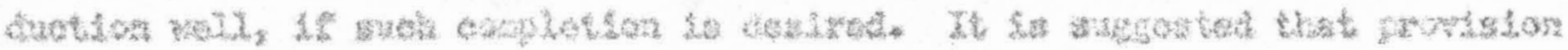

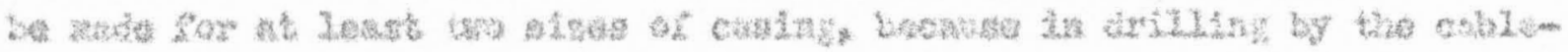

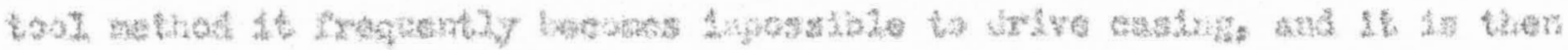

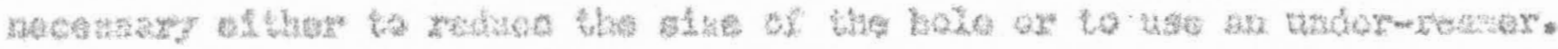

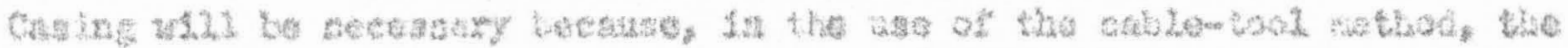

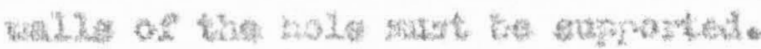




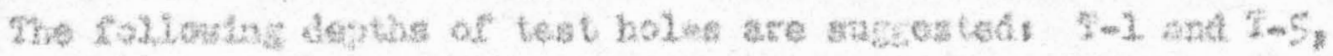

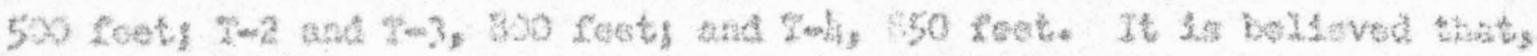

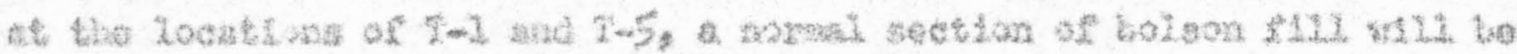

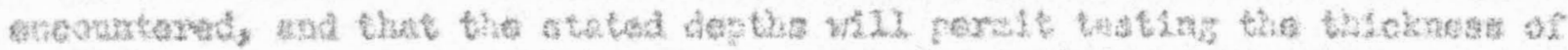

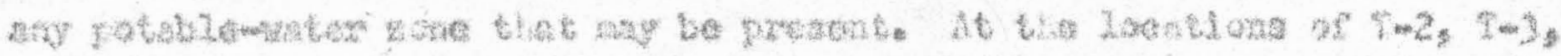

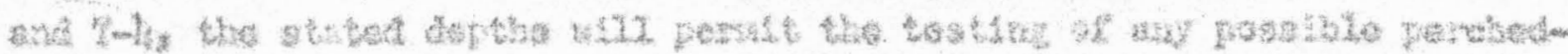

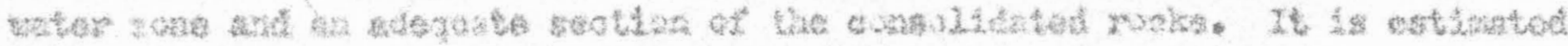

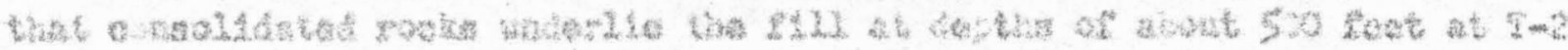

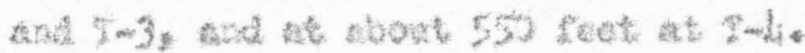

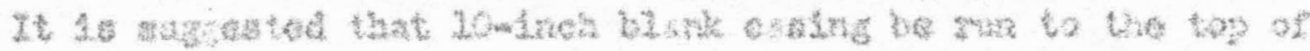

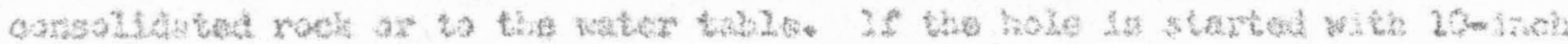

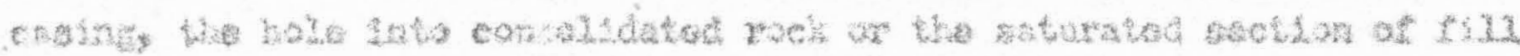

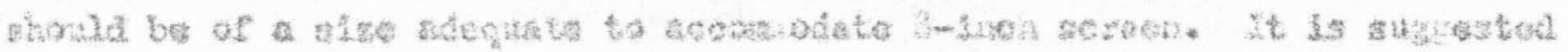

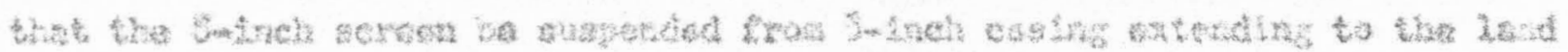

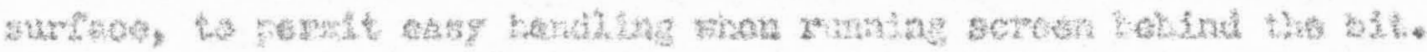

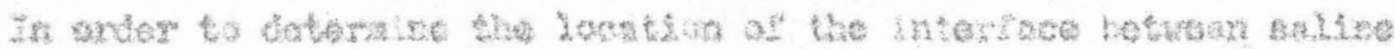

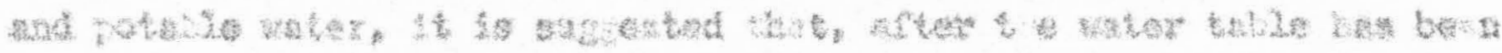

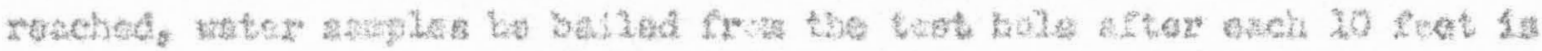

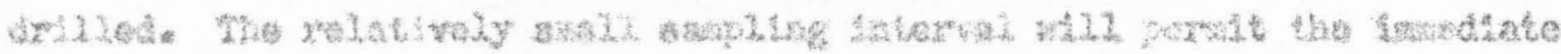

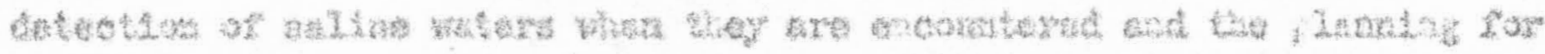

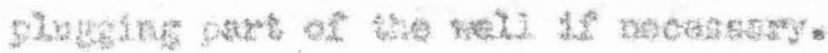

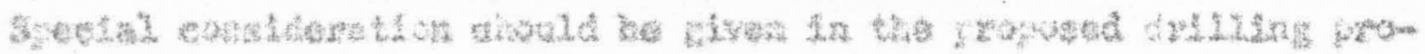

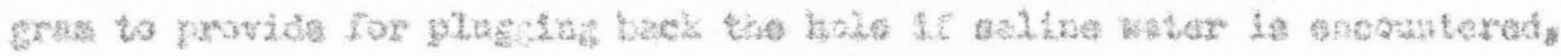

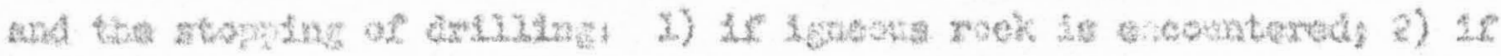

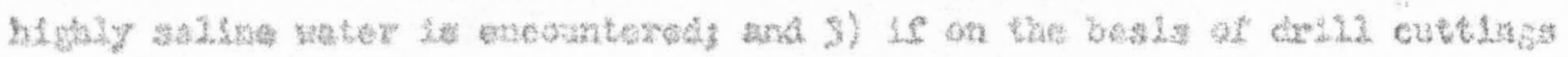

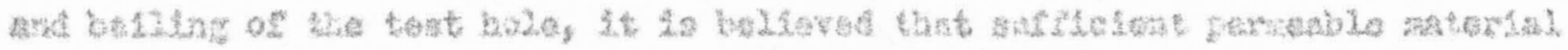

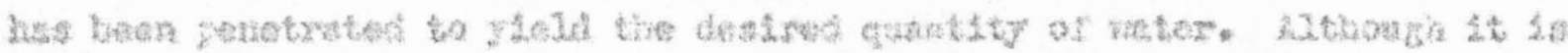




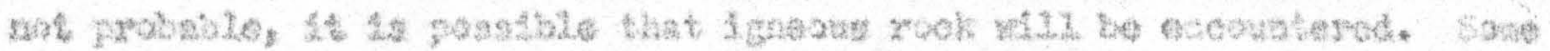

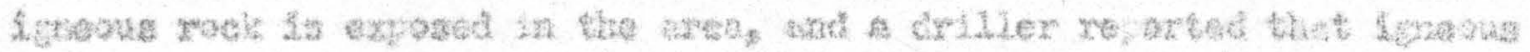

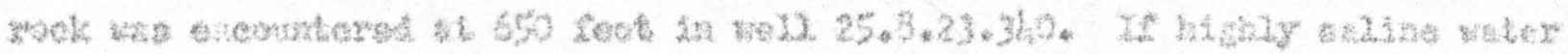

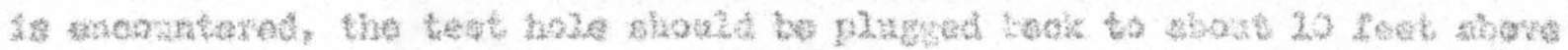

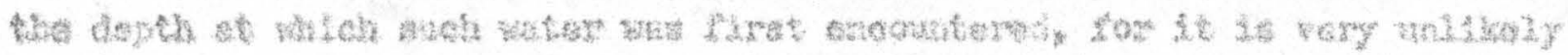

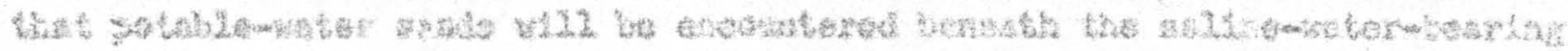
3

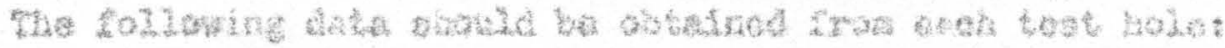

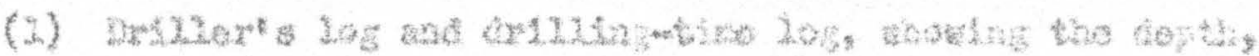

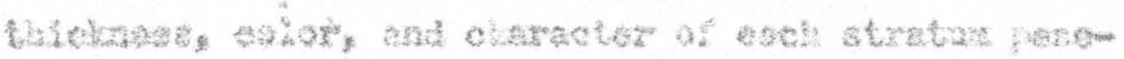

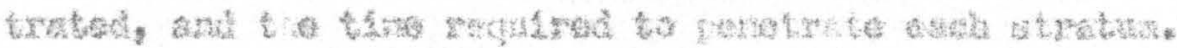

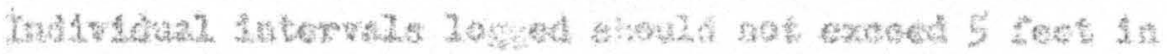
Straternot.

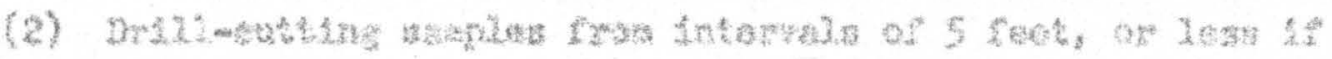

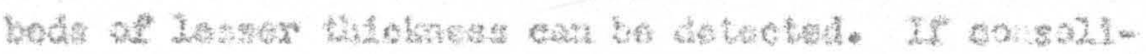

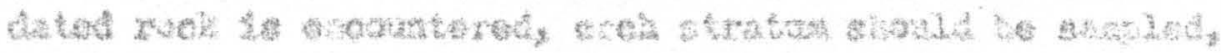

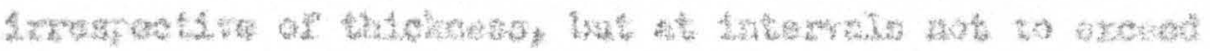

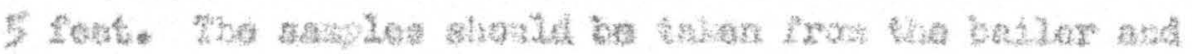
4hould to os

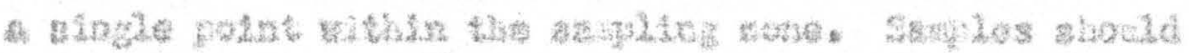

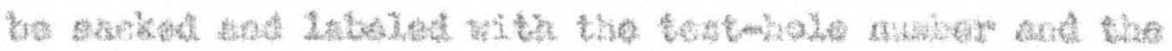

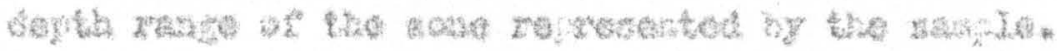




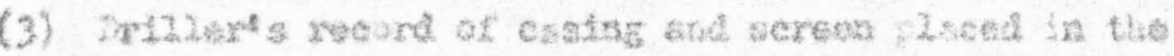
test Laite.

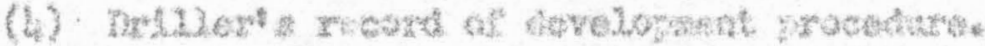

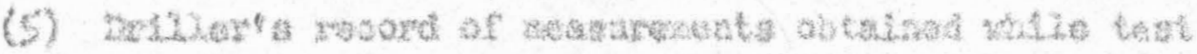
- puxatrot tho kelo.

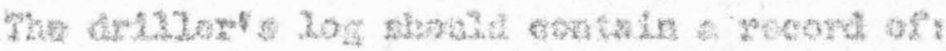

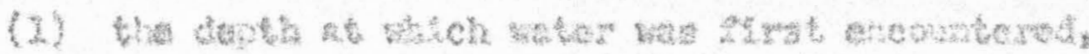

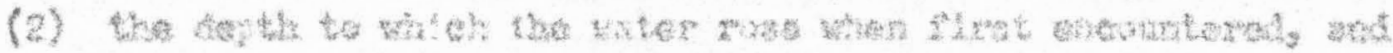

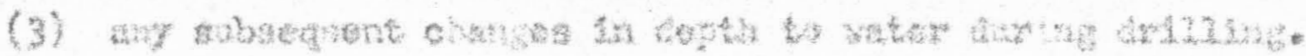

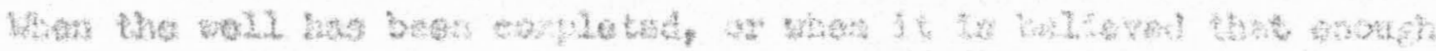

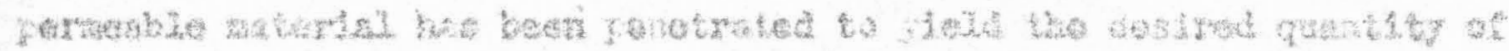

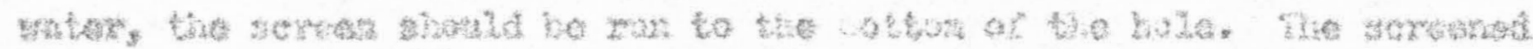

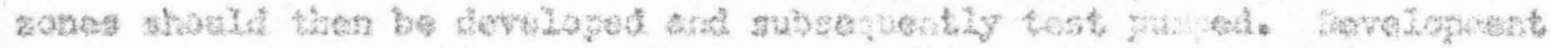

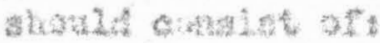

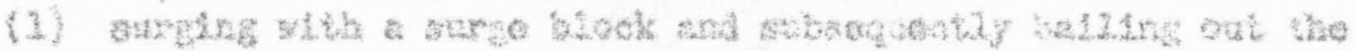

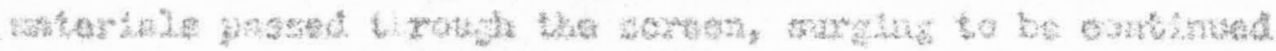

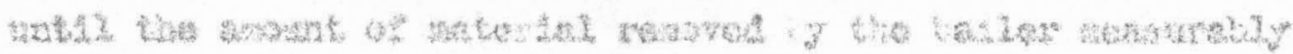
devatritshats

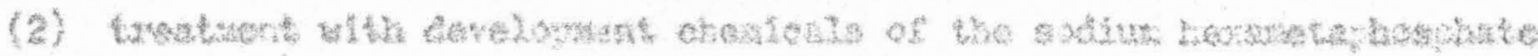
the the the of

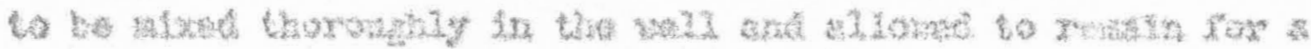

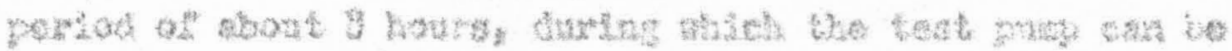
Asistanised

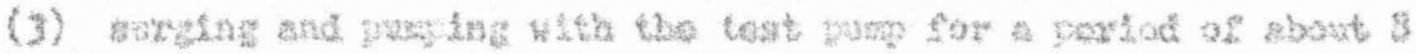

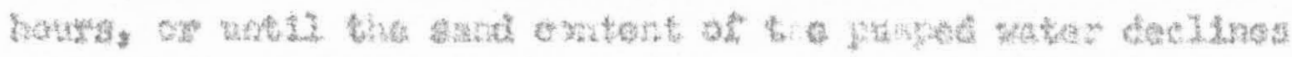

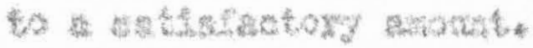




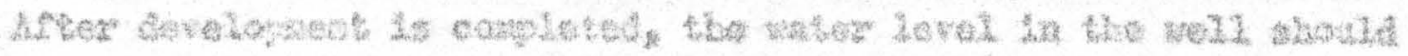

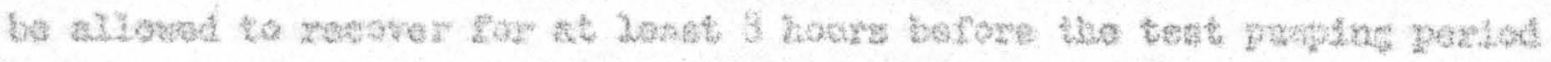

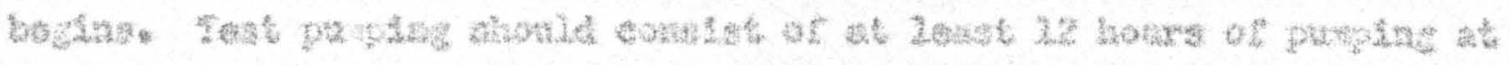

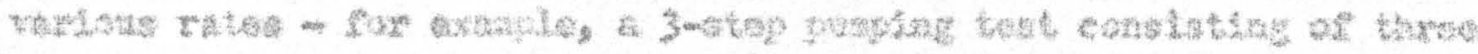

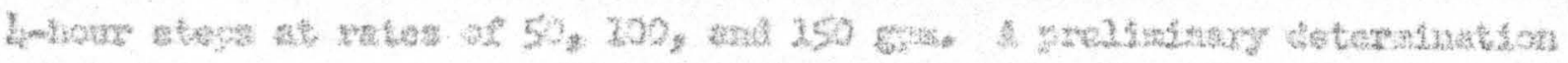

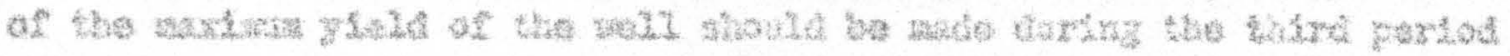

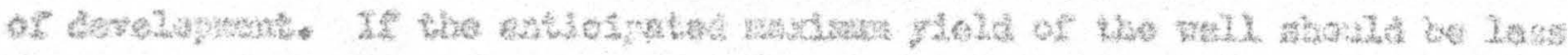

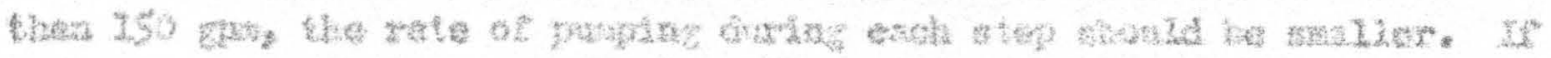

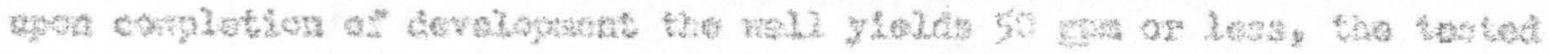

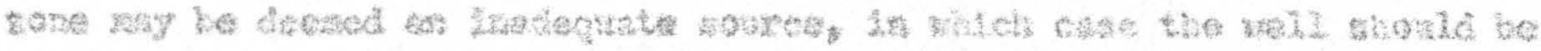

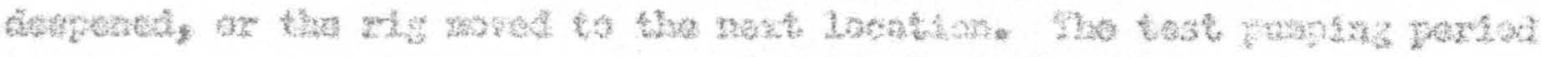

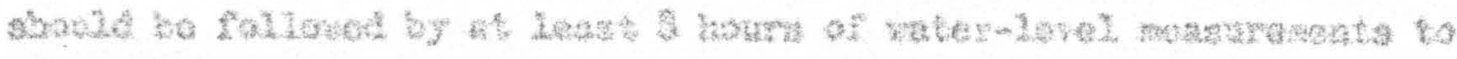

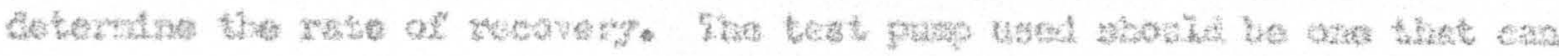

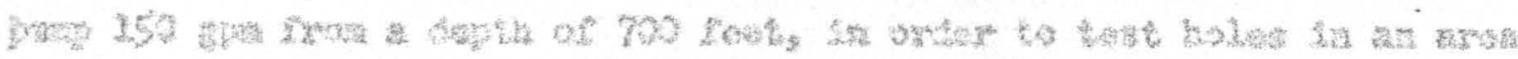

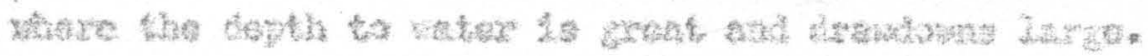




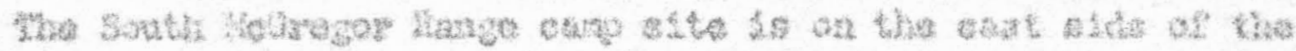

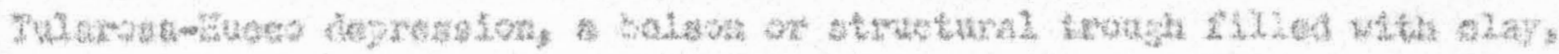

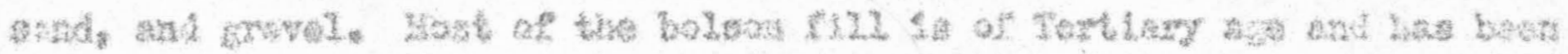
H. We

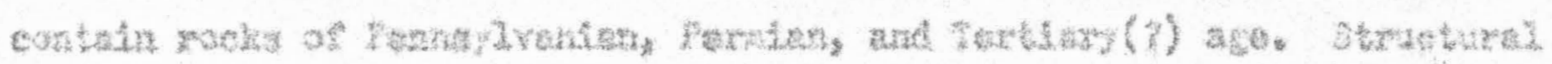

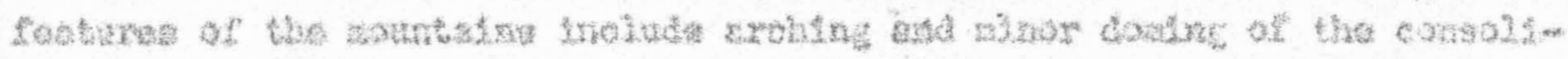

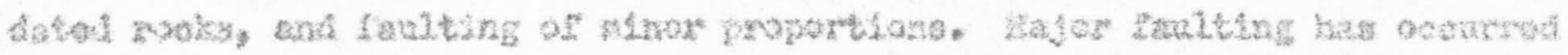

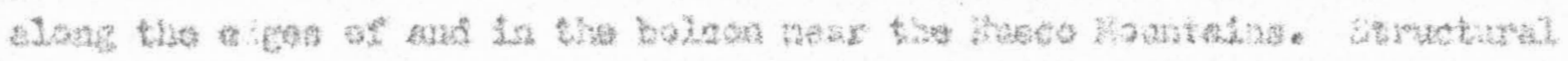

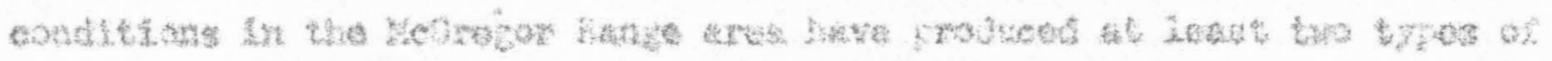

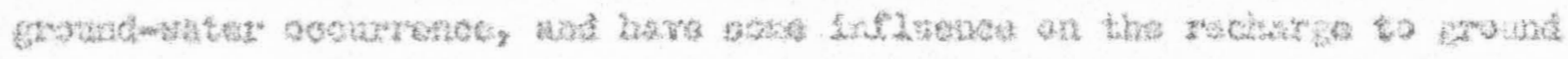

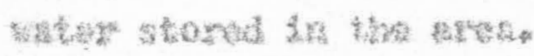

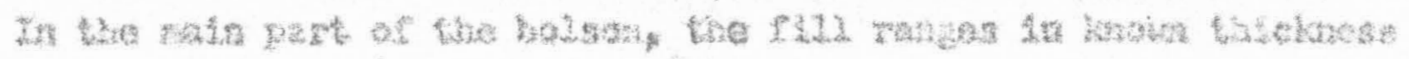

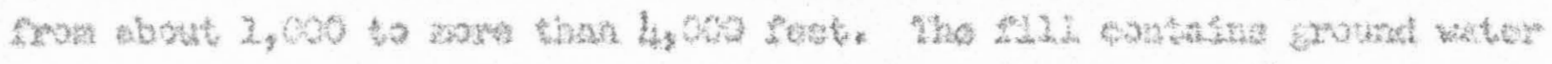

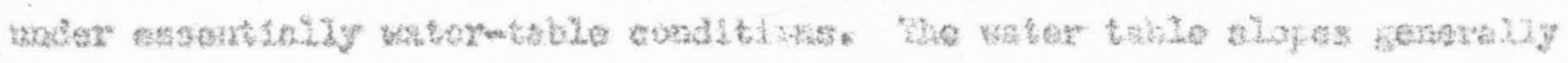

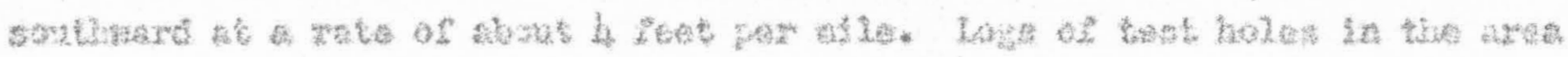

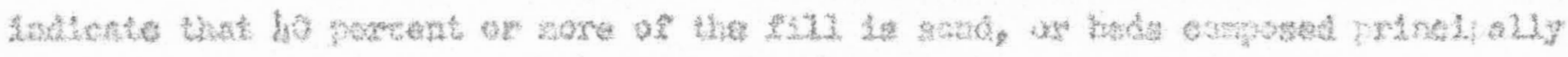

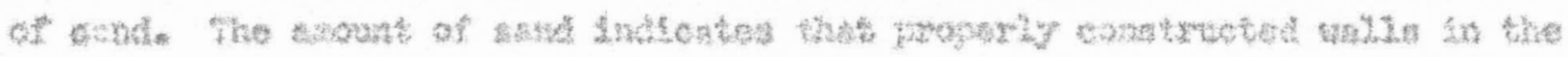

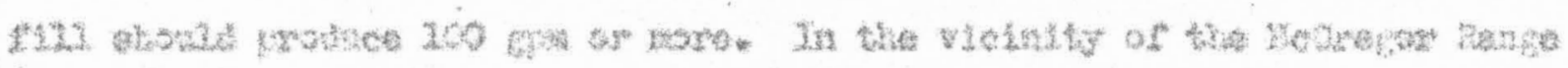

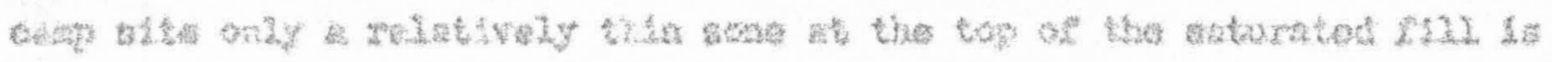

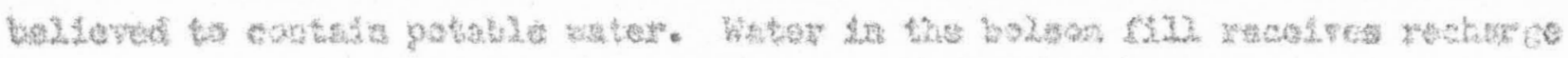

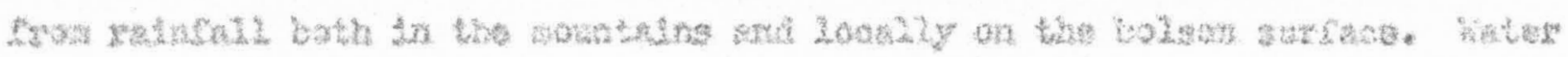

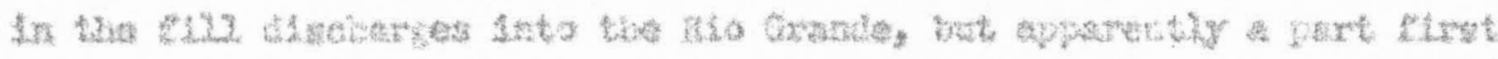

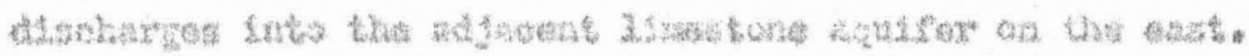




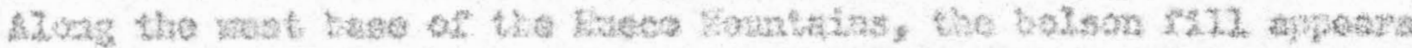

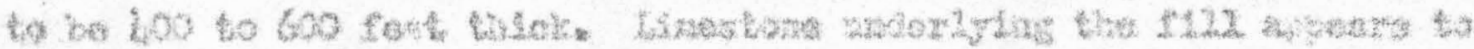

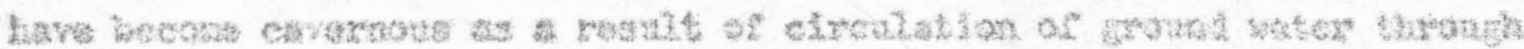

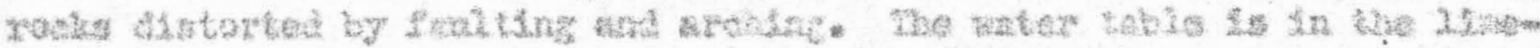

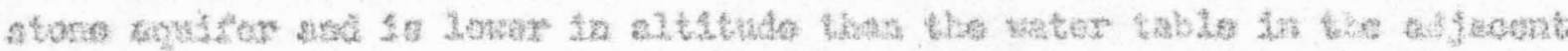

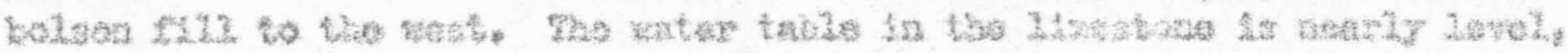

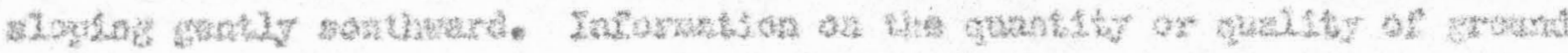

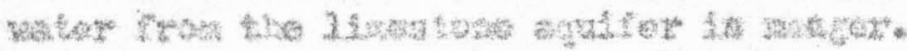

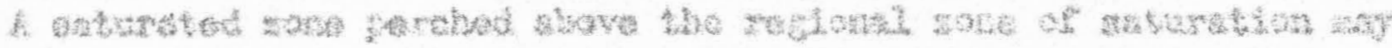

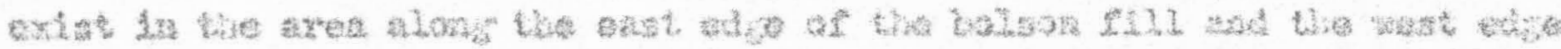

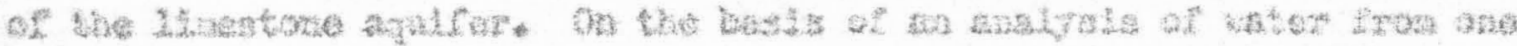

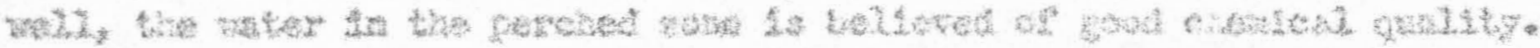

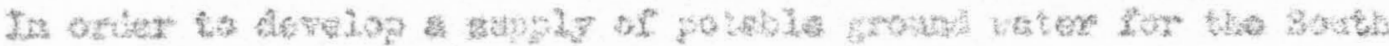

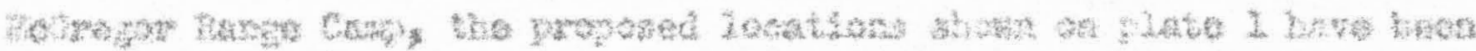

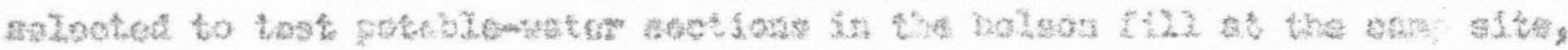
In

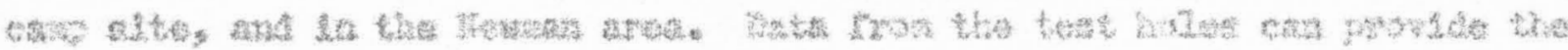

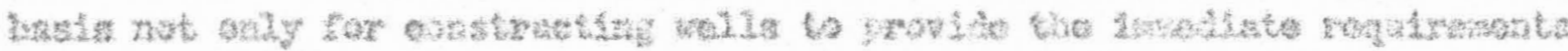

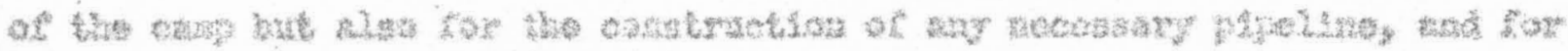

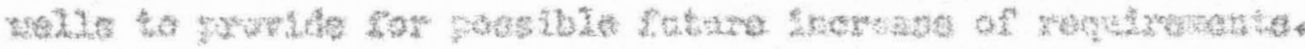




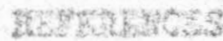

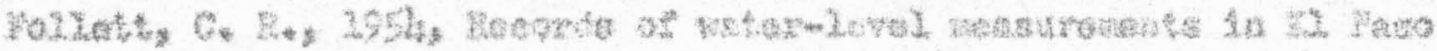

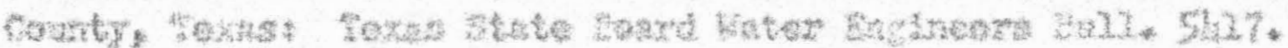

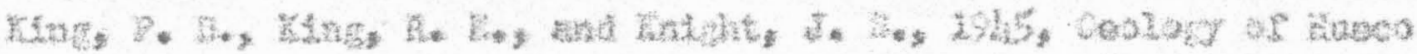

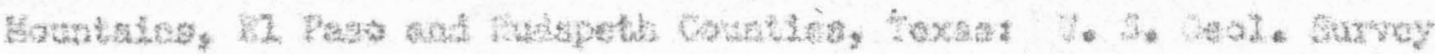

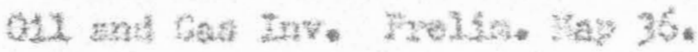

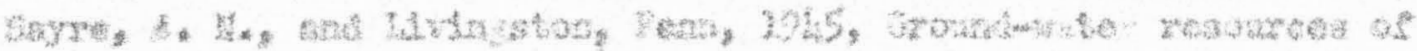

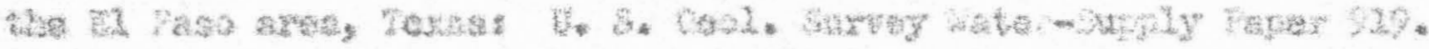

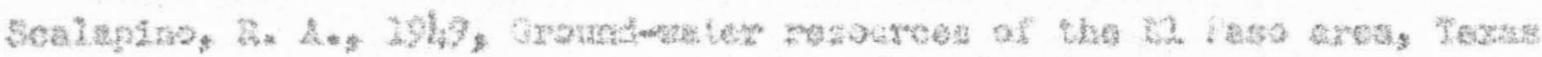
(ind

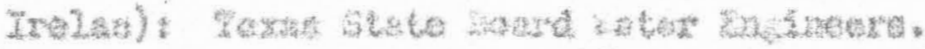

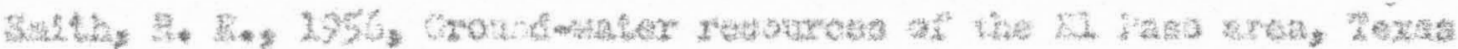

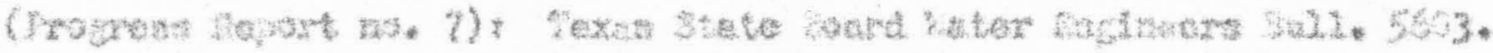


Table 2.--Records of wells and test holes in the vicinity of McGregor Range, Otero County, New Mexico, and El Paso Cuunty, Texas

\begin{tabular}{|c|c|c|c|c|c|c|}
\hline $\begin{array}{c}\text { Iocation } \\
\text { mimber }\end{array}$ & Ownex & Drizzer & $\begin{array}{c}\text { Date } \\
\text { drijied }\end{array}$ & $\begin{array}{l}\text { Depth } \\
\text { of woll } \\
\text { (feet) }\end{array}$ & $\begin{array}{l}\text { Diameter } \\
\text { of well } \\
\text { (inches) }\end{array}$ & $\begin{array}{l}\text { Altitude of } \\
\text { land surfaco } \\
\text { (feet) If }\end{array}$ \\
\hline & & \multicolumn{2}{|c|}{ Otero County, N. Mex. } & & & \\
\hline $23 \cdot 5 \cdot 35 \cdot 100$ & U. S. Govermmant & $B$. and $A$. Drilline Co. & 1953 & 650 & - & $4,080.7$ \\
\hline 24.7 .34 .244 & do. & do. & 1953 & $I, 209$ & - & $4,070.5$ \\
\hline 24.8 .1 .233 & - & - & old & 410 & - & $4,220 \mathrm{E}$ \\
\hline $24.8 \cdot 32 \cdot 344$ & State of New Mexico & B. and W. Drilling Co. & 1953 & 945 & - & $4,000 \mathrm{E}$ \\
\hline $25 \cdot 6 \cdot 4.170$ & U. S. Government & do. & 2953 & 1,208 & - & $4,060 \mathrm{E}$ \\
\hline $25 \cdot 6 \cdot 20.334$ & do. & Layne-Texas Co. & 1943 & 439 & $10,8,4$ & - \\
\hline $25 \cdot 5 \cdot 20 \cdot 334 a$ & do. & do. & 2944 & 440 & 12,20 & $4,029.4$ \\
\hline 25.6 .34 .111 & do. & B. and $W$. Drilline $C o$. & 1953 & 737 & - & $3,998.7$ \\
\hline
\end{tabular}


Table 2.--Reccris of wails and test holes in the vicintiy of lifuregor Fange, Otero Counity, N. isex., and El Paso County, Tex. - Continuea

\begin{tabular}{|c|c|c|c|c|c|}
\hline \multirow[b]{2}{*}{$\begin{array}{l}\text { Location } \\
\text { numiber }\end{array}$} & \multicolumn{2}{|c|}{ Water level } & \multirow{2}{*}{$\begin{array}{c}\text { Methid } \\
\text { of } 1 \text { ift } \\
2 i\end{array}$} & \multirow{2}{*}{$\begin{array}{c}\text { Jue of } \\
\text { water } \\
z \\
z\end{array}$} & \multirow[b]{2}{*}{ Dexaris } \\
\hline & $\begin{array}{c}\text { Belch iend } \\
\text { Eurface (feet) }{ }^{\prime}\end{array}$ & $\begin{array}{c}\text { Date of } \\
\text { measurement }\end{array}$ & & & \\
\hline & & & \multicolumn{3}{|c|}{ Ctcro Cennity, Now Hexion - Eontinued } \\
\hline $23.6 \cdot 35 \cdot 100$ & 207.5 & $\operatorname{mar} .29,1953$ & Ni & $\mathrm{Ii}$ & 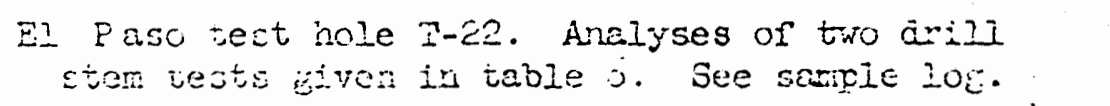 \\
\hline $24.7 \cdot 34 \cdot 144$ & 322.7 & $J u 2 y .14,2053$ & Ii & $N$ & $\begin{array}{l}\text { El Paso test koie } T-30 \text {. Analysis of one drill stem } \\
\text { test kiven in table ú. See sample iog. }\end{array}$ \\
\hline 24.8 .1 .233 & $.365 R$ & - & C,ir & ni & Inim as both Mott weII and as South well. \\
\hline $\begin{array}{l}24.8 .32 .3444^{2} \\
2518.6 .200\end{array}$ & - & - & $\because$ & Y & 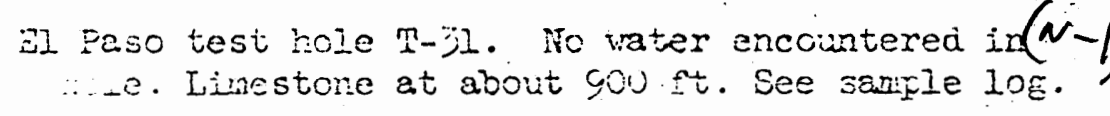 \\
\hline 25.5 .4 .110 & 300.0 & 1953 & II & $i_{i}$ & $\begin{array}{l}\text { II Faso test hole } \mathrm{i}-23 \text {. Analysis uf cne drill stem } \\
\text { test siven in table o. See sample los. }\end{array}$ \\
\hline $25 \cdot 6.20: 334$ & $300 R$ & 1943 & $9, G$ & TS & 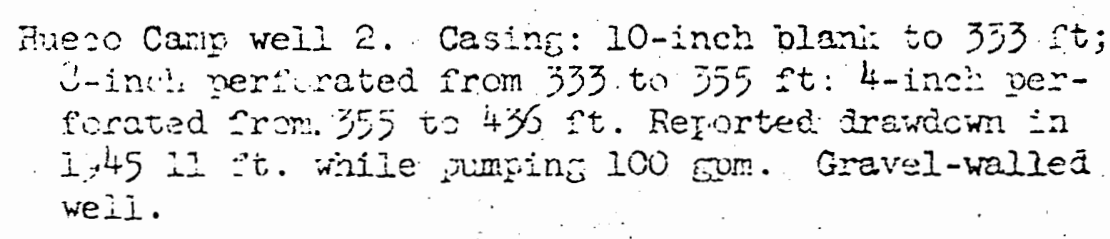 \\
\hline $25 . \therefore .20 .334 a$ & 203.02 & $\operatorname{Mar} \cdot 10,1554$ & $\mathrm{i}, \mathrm{C}$ & 29 & 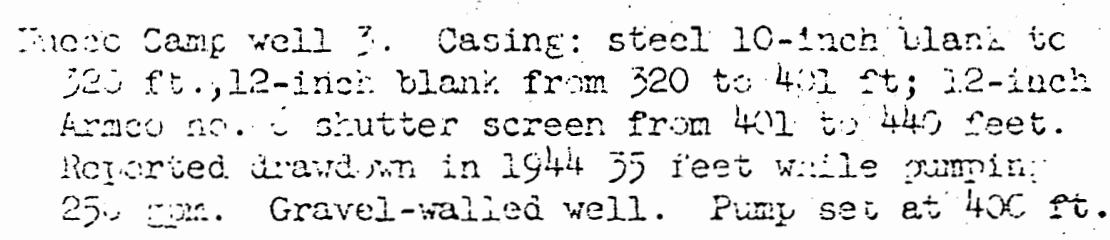 \\
\hline 25.6 .34 .121 & 270.0 & Apr.i7, $195 j$ & ii & $\mathrm{n}$ & $\begin{array}{l}\text { E- ino test nole T-30. Analyses of six arill stem } \\
\text { costs bren in tabie o. See sample log. }\end{array}$ \\
\hline
\end{tabular}


Thble 2.--Recoras of wells and test holes in tine vicinity of McGregor Range, Otero County, N. Wex., anc El Paso Cuuryy, Tex. - Conininued

\begin{tabular}{|c|c|c|c|c|c|c|}
\hline $\begin{array}{l}\text { Location } \\
\text { number }\end{array}$ & Owher & Drizler & $\begin{array}{c}\text { Date } \\
\text { cirilized }\end{array}$ & 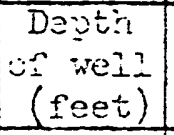 & $\begin{array}{l}\text { Dianeter } \\
\text { ci weil } \\
\text { (inches) }\end{array}$ & 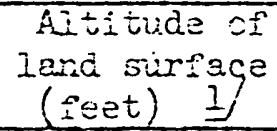 \\
\hline & & Otero County, N. liex. - & Ccntinued & & & \\
\hline ר 25.7 .15 .122 & State of llow Mexico & - & - & 441 & 8 & $4,101.4$ \\
\hline $25 \cdot 7 \cdot 20 \cdot 444$ & - & - & old & - & - & 1,098 \\
\hline 25.1 .24 .410 & State of New Mexico & - & 012 & 404 & 6 & $4,115 E$ \\
\hline-25.8 .1 .220 & do. & - & oid & 775 & - & $4,150 E$ \\
\hline 25.3 .23 .310 & do. & A] Parker & - & 1,000 & 8 & 4,2052 \\
\hline 25.8 .23 .540 & do. & do. & - & 700 & - & $4,220 E$ \\
\hline 25.8 .32 .220 & do. & - & oid & 785 & $\therefore$ & 4,1000 \\
\hline 25.3 .54 .420 & - & D. and w. Driline Co. & 1953 & $100-$ & - & $4,195 \mathrm{E}$ \\
\hline$-25 \cdot 9 \cdot 30 \cdot 340$ & State of ITeW Mexico & - & - & 2104 & 12,3 & $4,405 \mathrm{E}$ \\
\hline 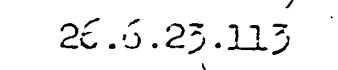 & - & - & - & - & - & $3,970.5$ \\
\hline 20 . 23.130 & Southern Pacific Lines & - & 1902 & 332 & 6 & - \\
\hline - $20.60 .34 .230 \mathrm{a}$ & do. & - & 2917 & $\therefore \quad 400$ & 18 & $3,998.2$ \\
\hline-25.7 .22 .122 & C. J. Mapel & - & 1550 & 450 & - & 4,0720 \\
\hline
\end{tabular}


Table 2.-Rezoris of relis and test holes in the vicinity of hichregor Rance,

Oterc Colinity, IN. Mes., and Il Paso County, Tex. - Jonitinued

\begin{tabular}{|c|c|c|c|c|c|}
\hline \multirow[b]{2}{*}{$\begin{array}{l}\text { Iccation } \\
\text { nuraber }\end{array}$} & \multicolumn{2}{|c|}{ Watur level } & \multirow{2}{*}{$\begin{array}{l}\text { Sietisoci } \\
\text { if } 1 ; i t\end{array}$} & \multirow{2}{*}{$\begin{array}{c}\text { ive of } \\
\text { Water } \\
3 i\end{array}$} & \multirow[b]{2}{*}{ Remarks } \\
\hline & $\begin{array}{c}\text { Below Iana } \\
\text { Surace (jeet) } 2 /\end{array}$ & $\begin{array}{l}\text { Datie of } \\
\text { neasurement }\end{array}$ & & & \\
\hline & \multicolumn{5}{|c|}{ Otoro County, Hew Mexios - Comtlmued } \\
\hline 25.7 .16 .122 & $\begin{array}{l}355.45 \\
354.79\end{array}$ & $\begin{array}{l}\text { Tuly } 5,1953 \\
\text { Niar. 23, } 1954\end{array}$ & $C, G$ & MS & $\begin{array}{l}\text { "Aivarudo well." Drilled to } 900 \text { feet (?). } \\
\text { Ectirated yield: } 15 \text { epmi. }\end{array}$ \\
\hline $2 j .7 .20 .444$ & - & - & $\because$ & ij & $\begin{array}{l}\text { Ermost io. 1. Ernest oil test. Reporied to have } \\
\text { entered Pennsylvanial rock at } 2,130 \text { feet. }\end{array}$ \\
\hline 25.7 .24 .410 & - & - & I. & ii & Unused stoci: weil. \\
\hline $25 \cdot 3 \cdot 1.22 c_{i}$ & .506 .70 & Jan.12,1950 & $\mathrm{C}, \bar{i}$ & $\mathrm{ij}$ & Unused stock well. "Borrepo tank well." \\
\hline $25 \cdot 8 \cdot 23 \cdot 310$ & - & - & IN & $\mathrm{N}$ & $\begin{array}{l}\text { Oil test. Drilier reports well entered Pennsyl- } \\
\text { vanian rocis st } 400 \text { feet. Very porolis limestone } \\
\text { at ilo teet. }\end{array}$ \\
\hline 25.8 .23 .740 & - & - & $\mathrm{H}$ & if & $\begin{array}{l}\text { Oil test. Driller reports i greous ricl: cncoun- } \\
\text { tered at } \quad j \neq 0 \text { feet. }\end{array}$ \\
\hline 25.3 .32 .220 & - & - & iv & ii & $\begin{array}{l}\text { "Fit tani: hell." Found in January } 1956 \text { to de } \\
\text { injed to ljl feet belor land surface. }\end{array}$ \\
\hline $25.8 \cdot 34.410$ & - & - & $\mathbb{N}$ & in & $\begin{array}{l}\text { El Paso test hole } T-38 \text {. Hole abandoned at less } \\
\text { than lou feet owing to loss of circulation, } \\
\text { and cavine. }\end{array}$ \\
\hline $25 \cdot 9 \cdot 30 \cdot 3 \div 0$ & - & - & ir & $\pi$ & $\begin{array}{l}\text { Well appears to be an old cil test. Double cas- } \\
\text { ing at land suriace in pit wilch may be cla } \\
\text { drilling "cellar". Hole dry and caved at } 210 \\
\text { feet, Ja.uary lo5i. }\end{array}$ \\
\hline 26.5 .23 .113 & - & - & C, it & $S$ & $\vdots$ \\
\hline 26.6 .34 .130 & - & - & $C, G$ & $\mathrm{RR}$ & Wu11 141 in Water Supply Paper 919. \\
\hline $26.5 .34 .130 \mathrm{a}$ & $\begin{array}{l}283.27 \\
283.4\end{array}$ & $\begin{array}{l}\text { July } 11,1938 \\
\text { Jan. } 7,1954\end{array}$ & $c, G$ & $R R$ & Viell 140 in Water Supply Peper 919. See loz. \\
\hline 25.7 .22 .122 & .325 .59 & June 23,1937 & $\mathrm{C}, \mathrm{H}$ & $\mathbb{N}$ & $\begin{array}{l}\text { Well } 152 \text { in Water Supoly Paper.919. Unused stodb } \\
\text { and domestic well. }\end{array}$ \\
\hline
\end{tabular}


Teble 2.--Records of wells and test hiles in the vicinity of Mccregor Range, Otero County, i. Hex., and El Paso County, Tex. - Continued

\begin{tabular}{|c|c|c|c|c|c|c|}
\hline $\begin{array}{l}\text { Locatton } \\
\text { muber }\end{array}$ & owner & Drilier & $\begin{array}{c}\text { Date } \\
\text { arilied }\end{array}$ & $\begin{array}{c}\text { Depth } \\
\text { of well } \\
\text { (feet) }\end{array}$ & $\begin{array}{l}\text { Diameter } \\
\text { of well } \\
\text { (inches) }\end{array}$ & $\begin{array}{l}\text { Altitude of } \\
\text { land surfage } \\
\text { (feet) If }\end{array}$ \\
\hline : & . & Otero Counity, iv. Mex. - & ontinued & & & \\
\hline $25.7 \cdot 32.122$ & State or New Mexico & B. and W. Drilling Co. & 1953 & 435 & $j$ & $4,051.2$ \\
\hline $26.7 \cdot 35 \cdot 230$ & do. & 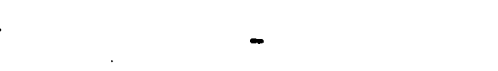 & ola & 400 & 5 & $4,077 \mathrm{E}$ \\
\hline 26.8 .8 .133 & $\therefore$ ao. a & - & $0: a$ & 450 & 8 & 4,1028 \\
\hline $20.8 \cdot 32.111$ & $\because \quad-$ & B. and W. Drilling Co. & 1953 & 824 & - & $4,094.6$ \\
\hline $26.8 .33 .120^{\circ}$ & Maver Bros. $\because$ & - & 012 & 500 & - & $4,095 \cdot 3$ \\
\hline $26.8 .33 .120 \mathrm{a}$ & $d z$ & - & old & - & - & $4,095.3$ \\
\hline & & El Paso Counity, Texas & & & & \\
\hline $1 \cdot 1 \cdot 5 \cdot 340$ & liavar Bros. & Payne and Jalierd & 1956 & $5: 24+$ & $8^{\circ}$ & $4,173 E$ \\
\hline 1.1 .5 .430 & $\therefore$ do. & - & 1952 & $500+$ & 6 & $4, I S C E$ \\
\hline .1 .73 .18 .130 & - & - & - & - & - & $4,073.4$ \\
\hline 1.79 .25 .340 & 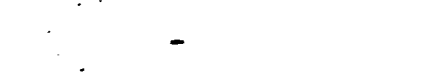 & - & - & - & - & $4,043.3$ \\
\hline 1.80 .1 .111 & U. S. Goverrment & B. and $v$. Drillin: Co. & 1953 & 801 & - & $3,994.7$ \\
\hline
\end{tabular}


Table 2.--Records of wells and test holes in the vicinity of NicGregcr Range,

Otero Cointiy, N. Mcx., and El Paso County, Tex. - Conitinued

\begin{tabular}{|c|c|c|c|c|c|}
\hline \multirow[b]{2}{*}{$\begin{array}{l}\text { Location } \\
\text { nimber }\end{array}$} & \multicolumn{2}{|c|}{ Nater level } & \multirow{2}{*}{$\begin{array}{l}\operatorname{detinda} \\
0+21+t \\
3 j\end{array}$} & \multirow{2}{*}{\begin{tabular}{c|} 
Use \\
water \\
$\overline{2} i$ \\
\end{tabular}} & \multirow[b]{2}{*}{ Pimaris } \\
\hline & $\begin{array}{c}\text { 3elow land } \\
\text { surface (feet) } 2 \prime\end{array}$ & $\begin{array}{c}\text { Date Cit } \\
\text { measurevient }\end{array}$ & & & \\
\hline & \multicolumn{5}{|c|}{ Oter, Cunty. ry isexic: - Continued } \\
\hline $2 \dot{c} \cdot 7 \cdot 32 \cdot 122$ & 347.92 & $\operatorname{Jan} .6,1354$ & is & iv & 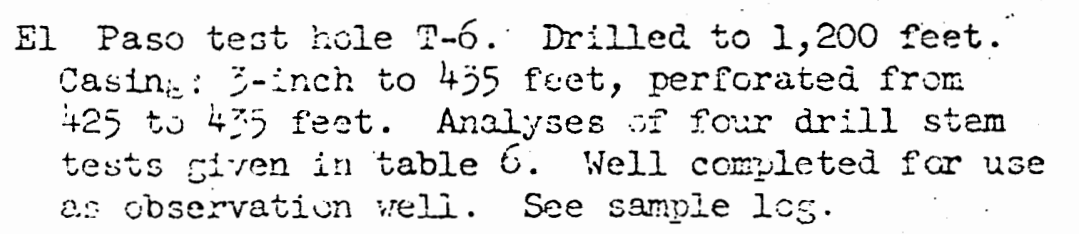 \\
\hline $26 \cdot 7 \cdot 35.230$ & 365.70 & July 19,1938 & iN & $i i$ & $\begin{array}{l}\text { "Jcint rell." Umeed stock well. Well } 153 \text { in } \\
\text { Water St: ily Paper } 919 .\end{array}$ \\
\hline 26.8 .8 .133 & - & - & 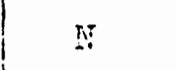 & iv & "rot well". Unused stock well. \\
\hline $20 . \Xi .32 .111$ & 447.3 & June 21,1953 & n & it & $\begin{array}{l}\text { Ei Paso test holo in. Anolyses of two drill stem } \\
\text { tot: itven in table } 6 \text {. See sample log. }\end{array}$ \\
\hline 26.3 .33 .120 & 162.0 & Ans. 9,1954 & $C, W$ & $S$ & Weil $15+$ in Water Supply Faper 919. \\
\hline $25.8 \cdot 3.320 a$ & 452.90 & Jily 19,1936 & H & iT & 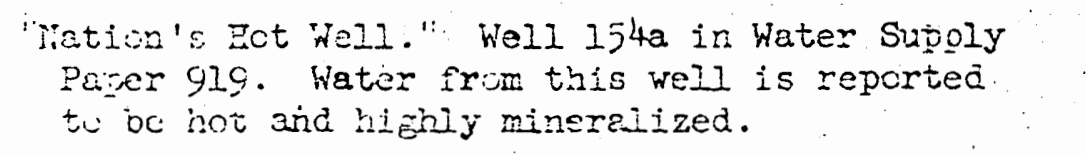 \\
\hline & & EI & Faso C & ty, & \\
\hline 1.1 .0 .7340 & - & - & - & - & $\begin{array}{l}\text { Neli vieited will driline in nrogrese. Projected } \\
\text { depth } 600 \text { feet. See partial log. }\end{array}$ \\
\hline 1.1 .6 .430 & $500+$ & 1952 & $\mathbb{N}$ & $\mathrm{IH}$. & Heil sound iu January 1956 to be filied. \\
\hline 1.73 .10 .130 & 365.95 & Apr. 14,1954 & $\mathrm{H}$ & in & "Waw Joint well". Unused stich well. \\
\hline $1.79 \cdot 10 \cdot 3+0$ & 337.54 & Arr. 14, 1954 & $\mathrm{N}$ & $\eta$ & Samel vell." Unused stoch well. \\
\hline 1.20 .1 .111 & 270.0 & Niar. 21, 1953 & $\mathrm{~N}$ & $\mathrm{ri}$ & $\begin{array}{l}\text { EI. Paso test hole } 9-4 \text {. Analyses of two drill stem } \\
\text { tests given in table } 6 .\end{array}$ \\
\hline
\end{tabular}


Table 2.--Records of wells ard test holes in i.e vicinity . YoGrosor Ranre,

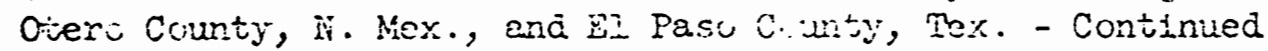

\begin{tabular}{|c|c|c|c|c|c|c|}
\hline $\begin{array}{c}\text { Location } \\
\text { number }\end{array}$ & Owner & Drtiler & $\begin{array}{c}\text { Date } \\
\text { driijed }\end{array}$ & $\begin{array}{l}\text { Deptr } \\
\text { of rell } \\
\text { (reet) }\end{array}$ & $\begin{array}{l}\text { Diameter } \\
\text { of weil } \\
\text { (inches) }\end{array}$ & $\begin{array}{l}\text { Altitude of } \\
\text { land surface } \\
\text { (feet) li }\end{array}$ \\
\hline & . & El Paso County, Tox. - & ontinued & & & \\
\hline 1.80 .4 .222 & - & B. and $\mathrm{w}$. Drilizine $\mathrm{Cr}$. & $1: 553$ & 520 & 3 & 4,0452 \\
\hline 1.80 .23 .444 & U. S. Govermment & - & C?a & - & - & 3.975 .1 \\
\hline 2.00 .24 .333 & do. & B. and $h$. Drilling $\mathrm{Co}$ & 1953 & 450 & 3 & $3,973.2$ \\
\hline
\end{tabular}


Tabie 2.--Recards of welis and tect joles in the vicinity cf NicGregcr Range, Otero County, IN. Mex., and EI Paso County, Bex. - Continued

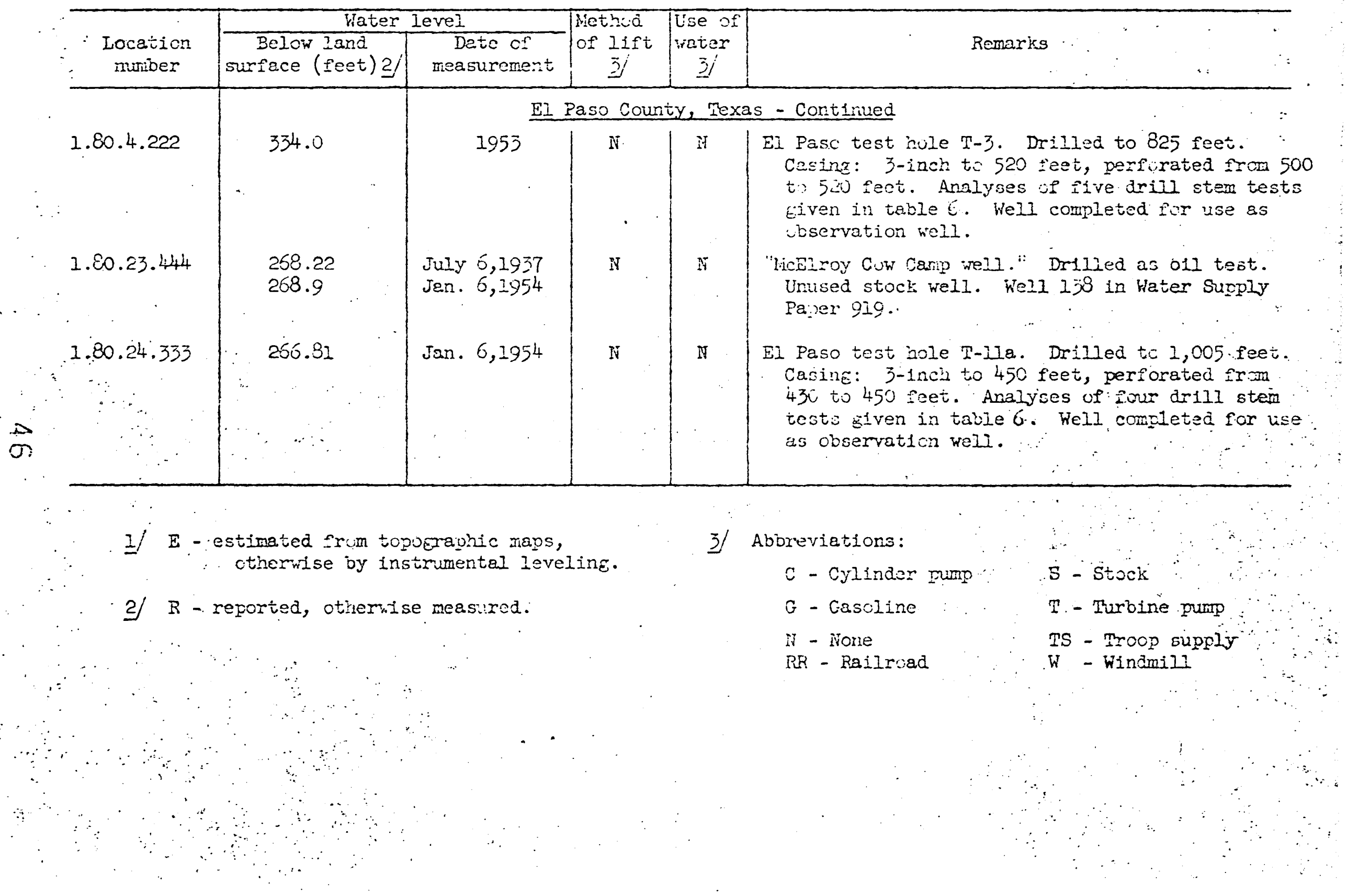


Trable 3.--Sample logs of wells and tost hules in vicinity of McGręor Ranze, Otero Cuunty, Nev Mexico

23.6.35.100 U. S. Guvernment

(E) Paso Test Hole 22, 1953)

\begin{tabular}{|c|c|c|}
\hline Naterial & $\begin{array}{l}\text { Tilinekness } \\
\text { (f'ect) }\end{array}$ & $\begin{array}{l}\text { Depth } \\
\text { (feet) }\end{array}$ \\
\hline Sand and caliche & 10 & 10 \\
\hline Medium-grained, red sand & 10 & 20 \\
\hline Fine to coarse-grained red sand & 10 & $\ddot{0}$ \\
\hline Fine to mearum-Grained red sand & 10 & 40 \\
\hline Fine-grained, red sand, and some caliche & 20 & 60 \\
\hline Very flue-trinined, red sand, and wome caliche & 10 & 70 \\
\hline Send anà gravel & 20 & 80 \\
\hline Clay and some sand & 10 & 90 \\
\hline Clay and Eravel & 10 & 100 \\
\hline Sand and cravel & 40 & 240 \\
\hline Fine-gralned sand, and clay & 10 & 3.50 \\
\hline Fine-grained sand & 10 & 160 \\
\hline Fine-Erained sand, a a d Eravel & 20 & 180 \\
\hline Fine to coarse-zralned sand & 13 & 3.93 \\
\hline Finc-grained sand, and clay. & 16 & 209 \\
\hline Fine to coarse-grained sand & 38 & 247 \\
\hline (Sarmile missine) & 3 & 250 \\
\hline Fine-treined sand & 20 & 260 \\
\hline Fine-grained sand, and clay & 1.0 & 270 \\
\hline
\end{tabular}


Iable 3.--Sample locs or wells and test heles in vicinity of McGreser Range, Otero County, M. Mex. - Contimed

23.5.35.100 U. S. Government

(EI Paso itest H.Ie 22, 195j)

-Continued

\begin{tabular}{|c|c|c|}
\hline Material & $\begin{array}{l}\text { Thicmess } \\
\text { (feet) }\end{array}$ & $\begin{array}{l}\text { Dept? } \\
(\text { roet })\end{array}$ \\
\hline Fine-errained sand, and clay and gravel & 19 & 289 \\
\hline Mediun-grained sana & 7 & 296 \\
\hline Fine to mediwn-eralned sand, ard cley & 4 & 31.0 \\
\hline Flne to mediuri-grified sand; claj and eravel & 20 & 300 \\
\hline Fine to medium-fratned sa! $a$ & 25 & 340 \\
\hline Sand and Gravel & 34 & 380 \\
\hline Sand, sravel and alay & 10 & 390 \\
\hline Fine-grained sard and eraval & 5 & 305 \\
\hline Very fine to fine-crit.:ed sand & 19 & 4.1 .4 \\
\hline Sand, cravel, and clay & i & 4.0 \\
\hline Fine to mediun-irsined and & 15 & 435 \\
\hline Fine to coarse-grained sand & 15 & 450 \\
\hline Fine to medium-irained wand & 18 & 468 \\
\hline Clay and fine-Erained sand & 12 & 480 \\
\hline Fine-Erained sand, and surle Gravel & 20 & 490 \\
\hline Fine-grained sand, some cravel and clay & 21 & 501 \\
\hline Fine-gralned sand, and clay & 9 & 510 \\
\hline Sandy clay & 20 & 520 \\
\hline Clay & 10 & 530 \\
\hline Clay and some gravel & 8 & 538 \\
\hline
\end{tabular}


Taule 3.--Sample Iogs $\mathrm{i}$ wolls and test holes in vicinity of MeGresor Rance, Otero Ccunty, N. Mex. - Continued 23.6.35.100 U. S. Gevernment (EI Paso Test H.Le 22, 1953) -Continued

\begin{tabular}{|c|c|c|}
\hline Materlal & $\begin{array}{l}\text { Thlciness } \\
\text { (fect) }\end{array}$ & $\begin{array}{l}\text { Depth } \\
\text { (feet) }\end{array}$ \\
\hline Sand, same grevel and clay & 2 & 540 \\
\hline Clay and some sand & 20 & $\cdots \quad 560$ \\
\hline Fine to medium-gralied sand & 12 & 572 \\
\hline Medtum-frained sand, a::d clay & 8 & 580 \\
\hline Clay & 5 & 585 \\
\hline Meatum-Fruined sand, and stme clay & .15 & 600 \\
\hline Fine te ciarse-urained cana & 20 & 610 \\
\hline Sand and clay & 20 & 630 \\
\hline Hedium to ccarse-erained sand & 10 & 540 \\
\hline Clay and sand & 10 & 850 \\
\hline
\end{tabular}


Table 3.--Sarule loes of wells and test holes in vicinity of MeCreger Ranie, Otero County, N. Mex. - Coniinued

24.7 .74 .100 U. S. Gevernment

(E.l Paso test hole 30,1953 )

\begin{tabular}{|c|c|c|}
\hline Material & $\begin{array}{l}\text { irficiness } \\
\text { (feet) }\end{array}$ & $\begin{array}{l}\text { Deoth } \\
\text { (feet) }\end{array}$ \\
\hline Sand, sowe sravel and calliche & 10 & 10 \\
\hline Fine to medium-gxained sand & 20 & 30 \\
\hline $\begin{array}{l}\text { Very flne to medium-Grafned sand, and } \\
\text { scrile clay }\end{array}$ & 10 & 40 \\
\hline $\begin{array}{l}\text { Very finc to medium-grained sand, and } \\
\text { some Gravel }\end{array}$ & 10 & 50 \\
\hline $\begin{array}{l}\text { Fine to cisise-fringed auk, and sone } \\
\text { calicho }\end{array}$ & 30 & 80 \\
\hline $\begin{array}{l}\text { Fine t. coarse-erained sand, sone caliche } \\
\text { and clay }\end{array}$ & 40 & 120 \\
\hline Clayay sand tu sandy clay and some caliche & 100 & 220 \\
\hline Silty to sandy clay and some caliche & 10 & 260 \\
\hline $\begin{array}{l}\text { Silty, plastic, daris-brom claj, some sand } \\
\text { and caliche }\end{array}$ & 50 & 310 \\
\hline $\begin{array}{l}\text { Hottled, reddish-brown, ard éray clay and } \\
\text { some caliahe }\end{array}$ & 90 & 400 \\
\hline Silty, medium-brown clay, sine sand a:d caliche & 90 & 490 \\
\hline Iicht tis mediun-brown clay aud some caliche & 100 & 620 \\
\hline $\begin{array}{l}\text { Redalsh-brim clay, mottied with eray clay; and } \\
\text { some caliche }\end{array}$ & 230 & 900 \\
\hline Silty, modium-urom clay and some caliche & & 1,209 \\
\hline
\end{tabular}


Table 3.--Samrle loce of vells and test holes in victnjty of NeGregir IRange, Otero County, N. Mex. - Continued

\section{$24.8 .32 .344^{2}$ state or New Mexioo}

(E]. Paso test role 3.1, 1953)

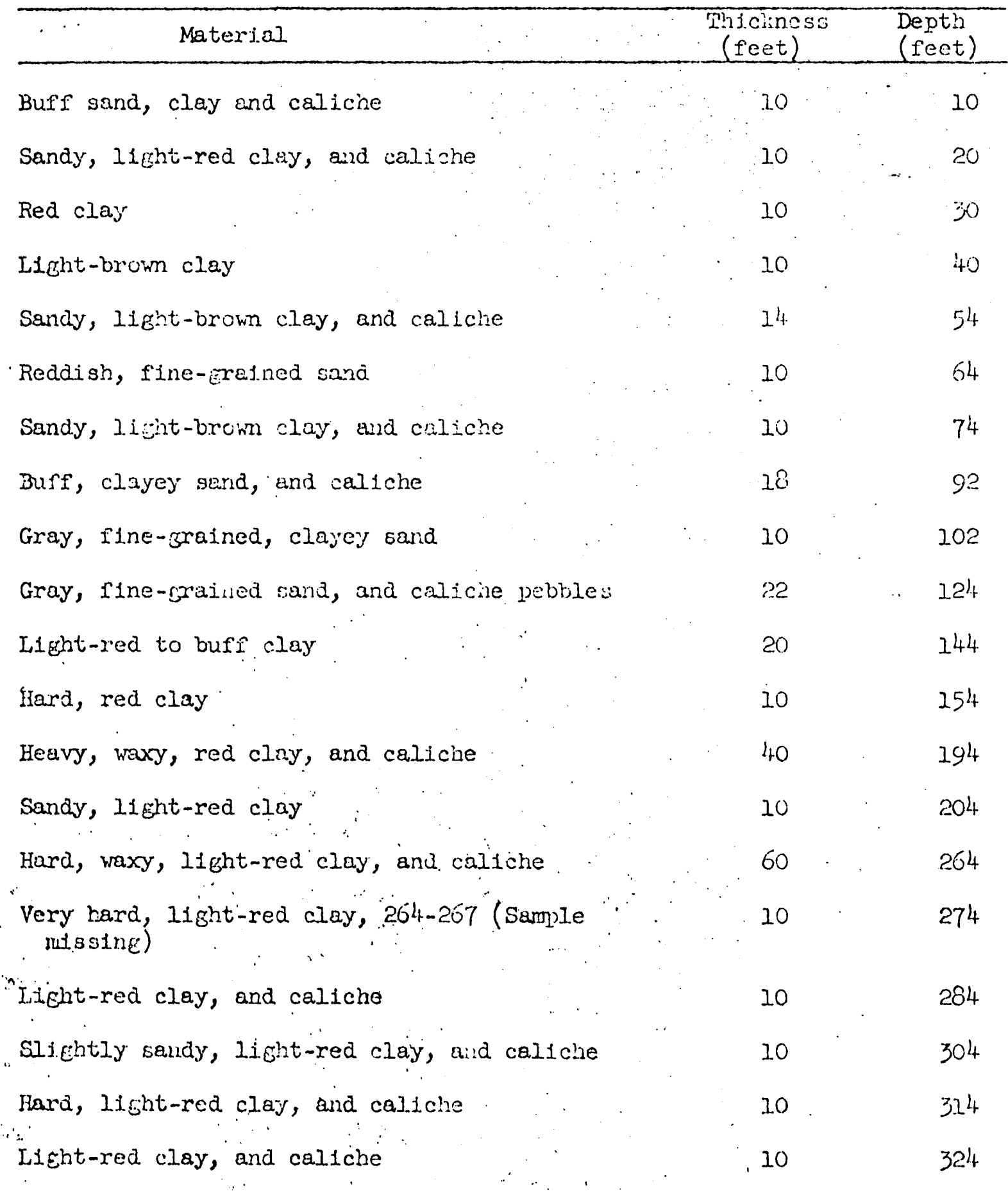


"iable 3.--Somple los of wisls and test holes in viciatity of

MeGrocer Range, Otero County, H.Mex. - Continued

24.0 .32 .344 State of Now Moxico

(El. Pa.jo test hole 31,1953 )

- Continued

\begin{tabular}{|c|c|c|}
\hline Naterial. & $\begin{array}{l}\text { Thiciness } \\
\text { (feet) }\end{array}$ & $\begin{array}{l}\text { Dents } \\
\text { (feet) }\end{array}$ \\
\hline Burf clay & 30 & $\therefore 354$ \\
\hline Lielt-rea slay, and calicile & 20 & $37^{4}$ \\
\hline Dars-red clay, and calicie & 10 & 334 \\
\hline Shaly, red olny, and coliche & 20 & 40.4 \\
\hline Red clay, and calicine & 10 & $i+24$ \\
\hline Fard, red elay & 50 & 4004 \\
\hline Sl.jehtily sandy, hara, red elay & 8 & 472 \\
\hline Hard, red clay & 32 & 504 \\
\hline Sli.ehtly sandy, red cluy & $\dot{0}$ & 510 \\
\hline Sendy, licht-red clay, and ralicie & 10 & 520 \\
\hline $\begin{array}{l}\text { Jight-reà clay, very fine-rined scindstine, } \\
\text { and caliche }\end{array}$ & 10 & 530 \\
\hline Sandy, li.gint-red clay, and calicine & 10 & 540 \\
\hline Sllghtly sandy, calcareous, lifht-red clay & 40 & 530 \\
\hline Sandy, calcureous, lifht-red and Gray clay & 24 & $60 \%$ \\
\hline Slizintly sandy, lient-red clay & 34 & 638 \\
\hline Buff to reddish-brum =lay & 232 & 770 \\
\hline Redaish-brown clay & 120 & 890 \\
\hline $\begin{array}{l}\text { (Sample missine - elcctric } 1 . j g \text { indicates limestine } \\
\text { was penetrated from about } 89 \text { ? to } 910 \text { fect) }\end{array}$ & 40 & 930 \\
\hline Clay and linestone cuttlngs & 15 & 945 \\
\hline
\end{tabular}


Table 3.--Semple low of wells and test hules in vicinfty of McGresur Range, Otero County, N. Mux: - Continued

22.6 .4 .100 U. S. Government

(E1 Paso lest kole 28, 195i)

\begin{tabular}{|c|c|c|}
\hline$\therefore$ Material & $\begin{array}{l}\text { Thiciness } \\
\text { (reet) }\end{array}$ & $\begin{array}{l}\text { Depin } \\
\text { (feet) }\end{array}$ \\
\hline Fine-rained wa:d & 20 & 20 \\
\hline Very fine-cralned sand, and clay & 20 & 40 \\
\hline Fine-eralined sand; Eravil and cal.tohe & 10 & 50 \\
\hline Sandy, buff clay & 10 & 6 \\
\hline Burs cla: & $1 \mathrm{k}$ & 100 \\
\hline Buff: elky, and sandstine & 40 & 140 \\
\hline Sandy, buff clay & 1.0 & 150 \\
\hline Bufi slay, and cuarse uravel & .22 & 152 \\
\hline Fine-ürain:s sand & 3 & 170 \\
\hline Bufr clay & 39 & 209 \\
\hline Fine-graincd sand & 19 & 228 \\
\hline Buff clay & 12 & 240 \\
\hline Medium-Erained sand & 10 & 250 \\
\hline Buff clay & 1 & 251 \\
\hline Medium-orained sand & 10 & $20 ́ 1$. \\
\hline Medium to vcry coarse-frained sand & 10 & 271 \\
\hline Flne-grained said & 20 & 291 \\
\hline Very rine-frained sand & 10 & 301. \\
\hline Very rine-crained sand, and Gravel & 20 & 321 \\
\hline Veryr sandy clay & 10 & 331. \\
\hline Very crarse-Erained sund, and clay & 10 & 341 \\
\hline
\end{tabular}


Table j.--Sample los of wells and test holes in vicinity of MeGrewr Rur e, Otero Cunty, N. Mex. - Continued 25.6 .4 .100 U. S. Govermment

(EL Pas: Lest liole 20, 195j)

- Concimad

\begin{tabular}{|c|c|c|}
\hline Material & $\begin{array}{l}\text { Theness } \\
(\text { f'ect) }\end{array}$ & $\begin{array}{l}\text { Denti } \\
\text { (feet) }\end{array}$ \\
\hline Saldy clay end an zilar pravel & 12 & 353 \\
\hline Sandy clay & $j$ & 355 \\
\hline Burf clay & 1.5 & 371 \\
\hline Clay ank sand & 10 & 3 בה \\
\hline Sard anú scric clay & $i j$ & 39. \\
\hline Fine-zrainea sand & 1) & 402 \\
\hline Medium-grained sand & 10 & $4 i i$ \\
\hline Nedium-araned sand, end clay & 10 & 1.2 .1 \\
\hline liedium-eratined saila. & 10 & 431. \\
\hline Fine to nedium-Eraired sar:d & 10 & $4+1$ \\
\hline Fine to llediun-craineci ca:da, a:d liay & 20 & WCI \\
\hline Mediun-jrained sand & 20 & 473. \\
\hline Sandy clay & 24 & 435 \\
\hline Bruwn clay and anelizar gavel & 10 & 495 \\
\hline Curse-grained, angular eravel; sand and clay & 10 & 505 \\
\hline Coarse-crained sand; clay and [rave]. & 30 & 51.5 \\
\hline Sandy clay & 15 & 531 \\
\hline Sandy clay, and very cuarse-brained eravcl & 10 & 541 \\
\hline Fine to mediun-cralned sanci, and some clay & 10 & 551 \\
\hline Sandy, iuff clay & 10 & 55.2 \\
\hline Sandy clay and cray clay & 10 & 571 \\
\hline Sandy, burf clay & .10 & 58 . \\
\hline
\end{tabular}


Table 3.--Sample logs of wells and test holes in vicinity of McGregor Rance, Otero Ccunty, N. Mex. - Contimed.

25.6 .4 .100 U.S. Goveriment

(E.l Paso test inje 20, 1953)

- Continued

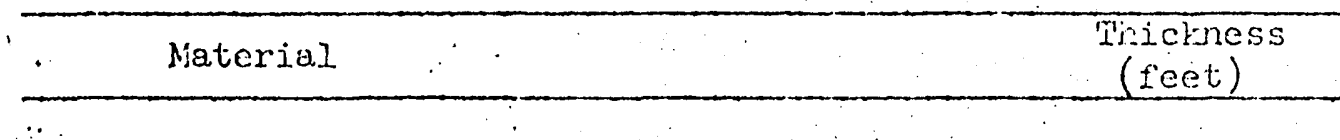

Sandy, buff clay, and caljiche pebiles

10

$5[1$

Buff clay and onie very fine-grained sand

10

601

Very fine to mediun-grainel sand, ard alay

10

6.1.

Very fine to modium-grained said, suò rraval

5

Cile

Buff clay, and sanci

20

530

Buff clay; sand" and gravel

10

645

Buff clay, and sand

20

565

Meülun-grained sanö; fravel and much clay

10

670

- Burf clay, ana sand

13

609

Buff clay, alld bentonite (?)

1.0

69c:

Sancy, burf clay

10

709

Buff clay, and mediun-ratned sana

10

719

Very fine-rrained sand, and clay

10

729

Fine to ccarse-erajned sand, and cjay

739

Very fine-Eralned sand, and clay

759

Very fine to coarse-grained sand, and nuch clay

20

763

Sandy, buff clay

10

773

Very fine-eralned sand, and clay

10

783

Buff clay and some and

25

808 
Table 3.--Sample logs of wells and test hules in vicinity of leGreear Rance, otero county, H. Miex. - Conitinued 25.6 .4 .100 U.S. Governuent

(El Paso teat hole 28, 1953)

- Cintinued

Buff clay

20

825

Buft clay and some fine-rrained sand

20

$8+3$

Medium-Eratned kand, and zJay

10

853

Buff cluy

10

¿603

Buff clay, aud medilini-grajned sard

$2: 3$

883

Fine to mediun-grafned sand, and ciay

10

808

Fine-graineci cand, anj clay

1.0

908

Very fine to mediul-frained sand; olay and

10

918 oravel.

Sandy, buif clay

10

926

Very fine-frained send, ard clay

10

933

Very fine-eraincd salid

10

9he

Very fine-erained saik, and clay

10

958

Clay and verg fine-eralned sand

10

963

Very fine-Graineà sand, and clay

20

988

Clay and rery fine-grained wand

40

1,028

Clay, very rine ti coarse-gralned sand, and sravel

10

$1,0: \hat{3}$

Brown clay.

10

1,040

Brown clay, aid fino-crained gravel

33

1,036

Buff clay, and fine-rained sand.

10

1,096

Brown claj, ILne-crained sand and pravcl.

9

1,105 
Pable 3.-Sample logs of wells and tast holes in vicinity of MoGregor Range, utoro County, li. hex. - Continued

23.6 .4 .200 U.S. Government

(El Paso test sule 28, 1953)

- Continued

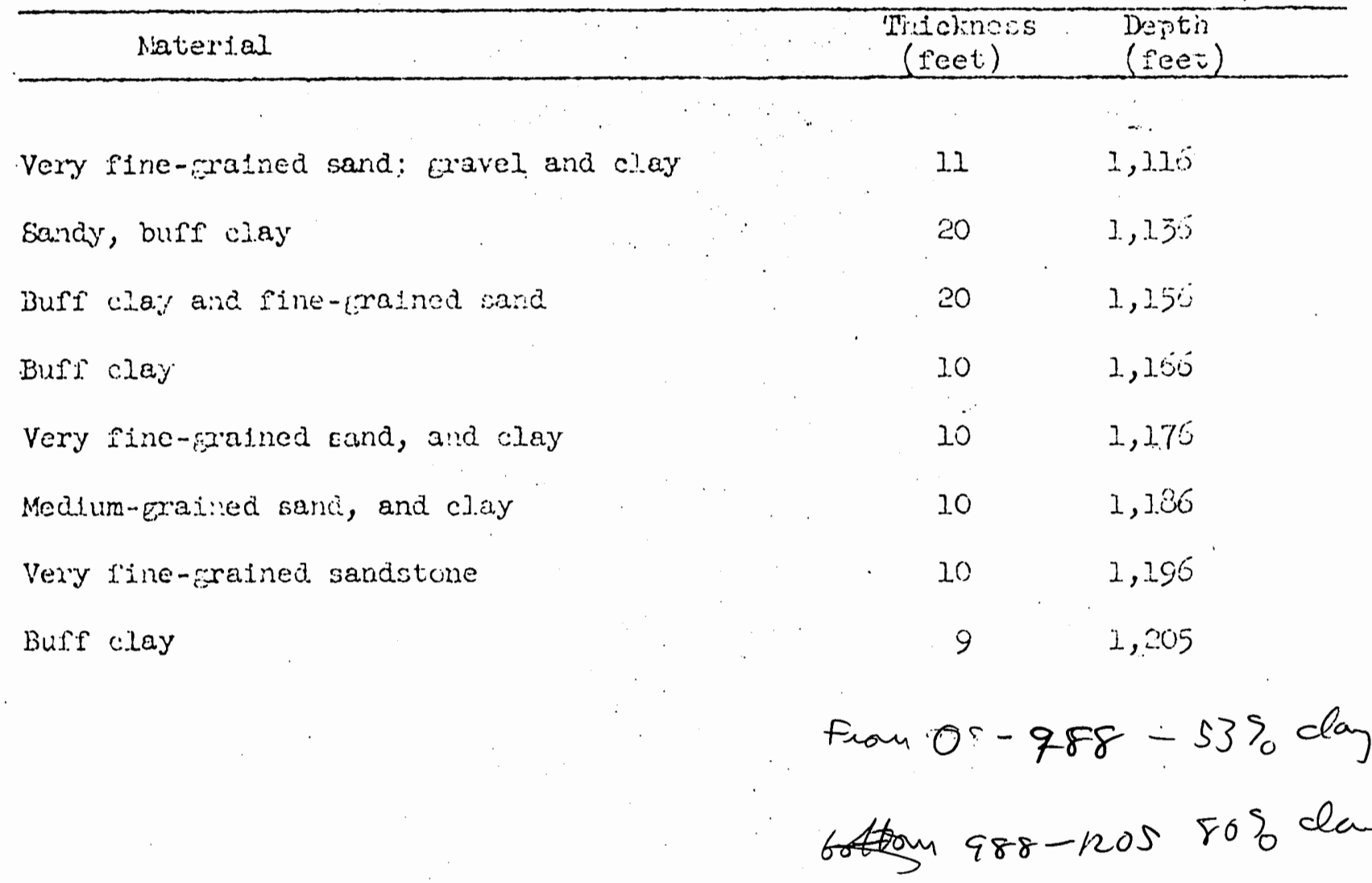


Tabie 3.--Samise logs of wells and test holes in vicinity of McGrecor Rance, Otcru County, N Mex. - Contimed 25.6 .34 .1 .11 U. S. Govermnent

(E] Paso teot hile 3i, 1953)

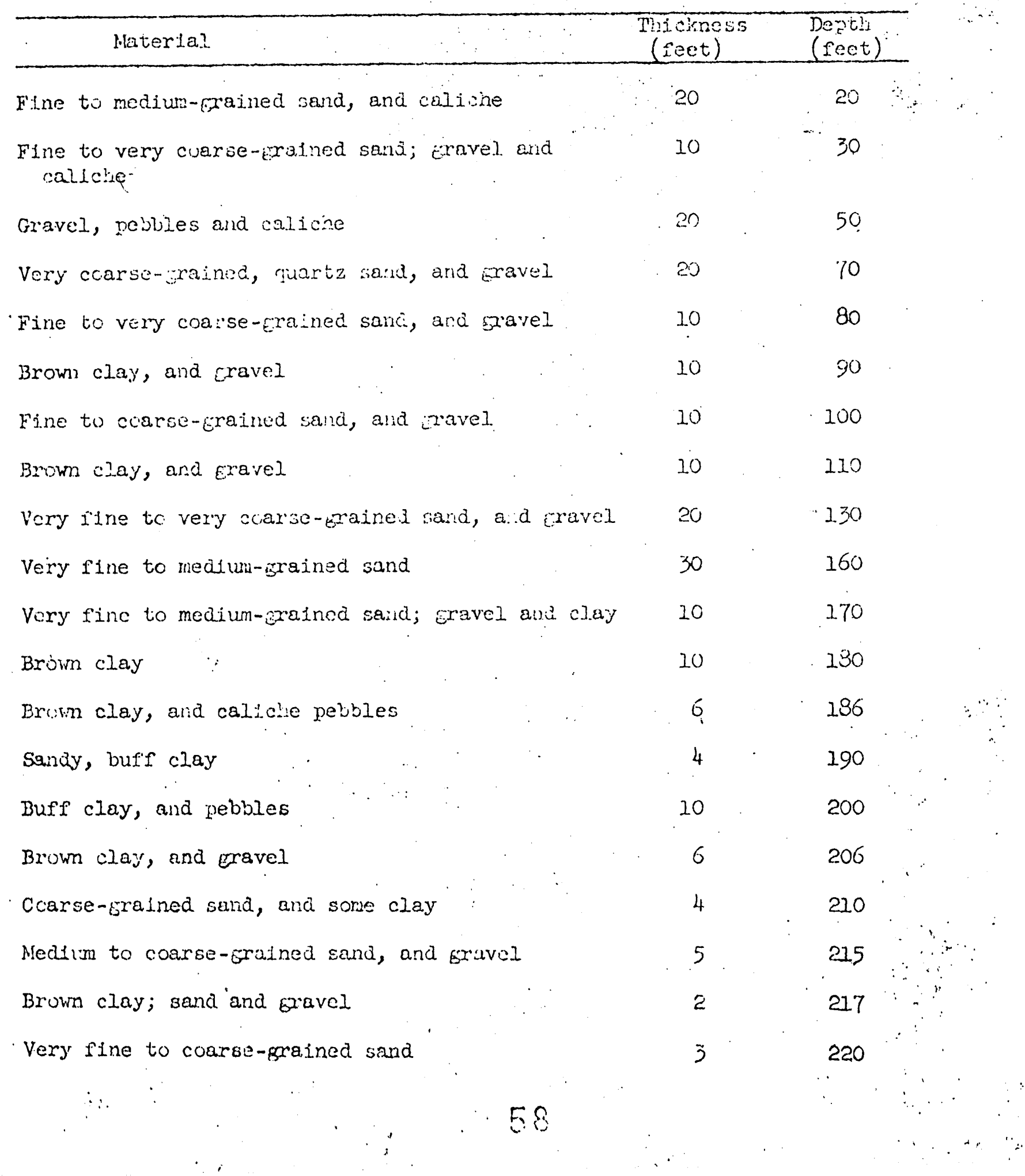


Table 3.--Sample logs of wells and test hules in vicinity af MicGrecror Range, otero County, N. Mex. - Continied 25.6 .34 .21 .2 U. S. Givernment

(El Paso test hole 30, 1953)

- Continued

\begin{tabular}{|c|c|c|}
\hline Miterial & $\begin{array}{c}\text { Thiomers } \\
\text { (feet) }\end{array}$ & $\begin{array}{l}\text { Deptin } \\
(\text { feet) }\end{array}$ \\
\hline Fine to very cuarse-crathed sand, and gravel & 10 & 230 \\
\hline Fine to coarse-Grained sanä; sravel and ciay & 10 & 240 \\
\hline Buff clay, and coarie-preined gravel & 24 & $25)_{4}$ \\
\hline Coarse to very cuarse-rnined sand, and clay & 巨 & 20 \\
\hline Bufr clay, and peobles & 10 & 20 \\
\hline Coarse to very ccarse-grainea salid, aida sme clay & 10 & 230 \\
\hline Sharp or ancular gravel, anà cail zhe & 10 & 290 \\
\hline Sandy, butes clay & 10 & 300 \\
\hline Angular eravel, and scre clay & 20 & 310 \\
\hline Very fine to medruin-praincd bliff sand, and clay & $\dot{u}$ & 315 \\
\hline Buff clay & 4 & 320 \\
\hline Broum to buff clays, and caliche & 10 & 330 \\
\hline Fine to ccarse-grained sand & 6 & 300 \\
\hline Buff clay & 4 & 340 \\
\hline Buff clay, and sravel & 5 & 346 \\
\hline Fine-crained sand & 7 & $35 \%$ \\
\hline Buff clay & 3 & 356 \\
\hline Medjum-cralned sand & 4 & 360 \\
\hline Buff clay, and cuarse-irainod cravel & 20 & 370 \\
\hline Buff clayr & 20 & 390 \\
\hline
\end{tabular}


Table 3.--Sample logs of wells and test boles in vicint by of MeGregor Rance, Otaro County, iN. Mex. - Ccntinued

25.6 .34 .111 U. S. Government

(El Faso tesu nule $\%, 1953$ )

- Continuea

\begin{tabular}{|c|c|c|}
\hline Natertal & $\begin{array}{l}\text { Tictiness } \\
\text { (leet) }\end{array}$ & $\begin{array}{l}\text { Deet: } \\
\text { (fest) }\end{array}$ \\
\hline Modium-zaincd sand & 10 & 400 \\
\hline Very fine to fine-grained sand & 10 & 410 \\
\hline Fine-frained sand; EraveI and aliche & il & 421 \\
\hline Fine to mediull-Grainsd sand, 3 al ajtche & 29 & it 40 \\
\hline Very fine to fine-grained sand & II & 451 \\
\hline Sandy, buff clay, and caliche & 9 & $1+60$ \\
\hline Sandy, buff clay; cravel, ard caliche & 10 & 470 \\
\hline Very fine to fine-crafied sand, and clay & 10 & 480 \\
\hline Sandy, buff clay, anà caliche cravel & 10 & 490 \\
\hline Buff clay, coarse-grained sand, and Eravel & 10 & 500 \\
\hline Very coarse-Erained sanä; gravel aid caliche & 20 & 520 \\
\hline Very fine to fine-irainea sand & 10 & 530 \\
\hline $\begin{array}{l}\text { Mediun to ccarse-crained sand; cravel, and } \\
\text { caliche }\end{array}$ & 12 & 542 \\
\hline Fine to coarse-crained sand; and caliche gravel & 18 & 560 \\
\hline $\begin{array}{l}\text { Medium to coarsc-crained sand; Gravel, callche, } \\
\text { and clay. }\end{array}$ & 20 & 570 \\
\hline Sandy, burf clay; aravel, and callcite sravel & 10 & 500 \\
\hline Clay, crarse-fralned sand, and gravel & 5 & 585 \\
\hline Fine to coarge-graincd said, and gravel & 10 & 595 \\
\hline Very sandy clay, nd ëravel & 10 & 605 \\
\hline 60 & & . \\
\hline
\end{tabular}


lable 3.--Sanple lors of wells and test holes in vicinity of McGrezor Range, Otero County, N. Mex. - Continued $25 \cdot 6.34 .111$ U. S. Goverrment

(El Paiso tect hole 36, 1953)

- Continued

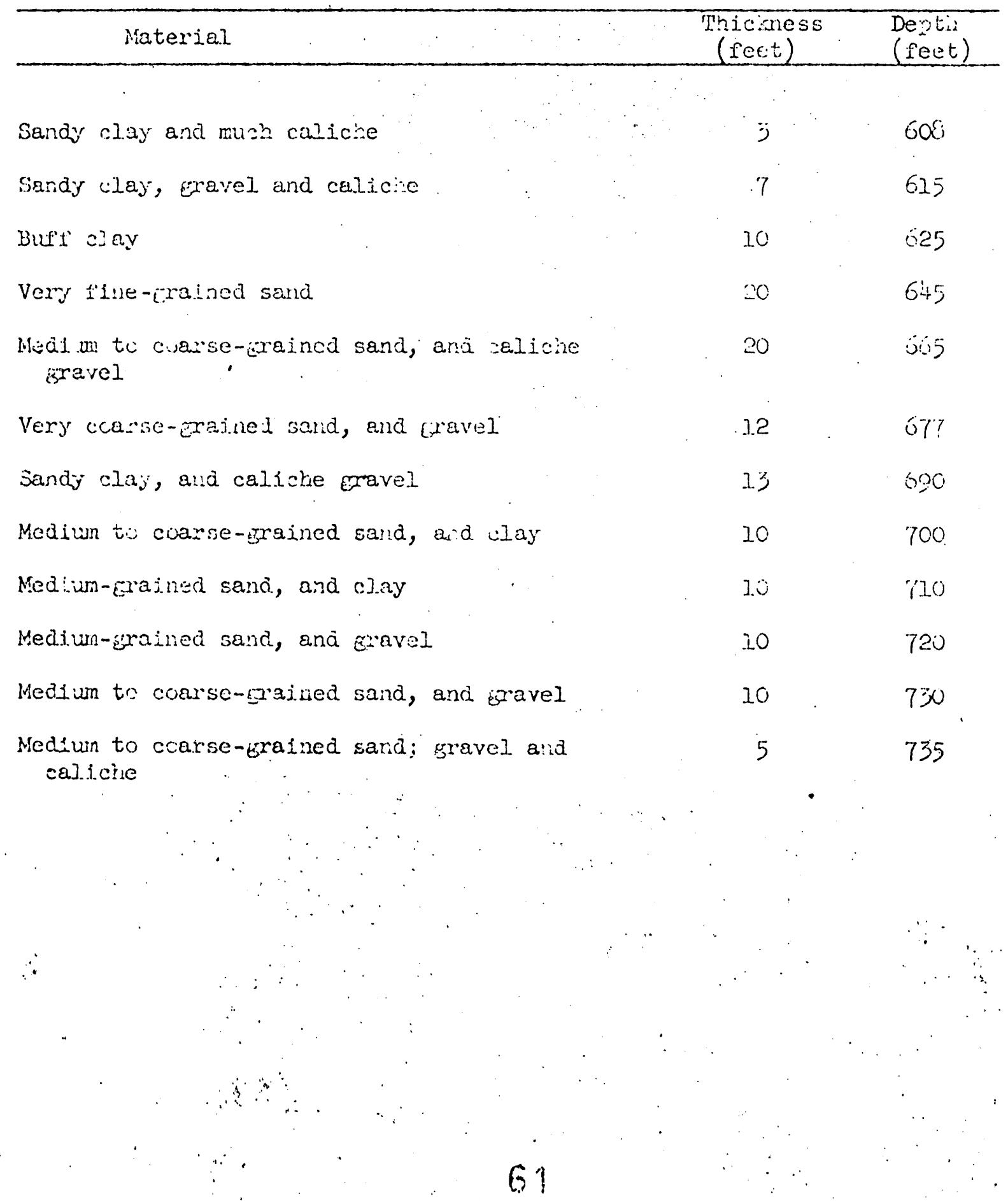


Table 3.--Sample logis df wells and test holes in viclnity of MaGragor Range, Ctero County, M. Mex. - Continued

26.7 .32 .122 State of New Mexiow

(EI Pasi test hole ó, 1.953)

\begin{tabular}{|c|c|c|}
\hline Material & $\begin{array}{l}\text { Thiciness } \\
\text { (feet) }\end{array}$ & $\begin{array}{l}\text { Dept? } \\
\text { (feet) }\end{array}$ \\
\hline$\because \quad \quad$ & 20 & 20 \\
\hline Coarse-grained fravel, red clay and caliche & 30 & 50 \\
\hline Ctarse-grainea anguler gravel, and calici:e & 1.0 & 60 \\
\hline Brown glay, and saliche & 10 & 70 \\
\hline Stunar, brown clay, and caliche & 1.3 & 30 \\
\hline Brown clay; sand and calishe & 20 & 100 \\
\hline Vory cciars-Erainea, angular kravel & 20 & 120 \\
\hline Fine-zainca rea sand & 2 & 122 \\
\hline Ccarse-crained sand; clay, and calicie reviles & 0 & 130 \\
\hline Calishe & .20 & $140^{\circ}$ \\
\hline Clarse to remy coarse-craincd ianu & 10 & 150 \\
\hline Brown clay, and caliche & 10 & 160 \\
\hline $\begin{array}{l}\text { Vory fine to very coarse-eralned said and } \\
\text { ccarse-galned, anejlar gravul }\end{array}$ & 30 & 190 \\
\hline $\begin{array}{l}\text { Very fine to coarse-grained sand, cuarse-grained } \\
\text { angular pravel, and caliche }\end{array}$ & 20 & 200 \\
\hline Sandy, burf clay & 20 & 220 \\
\hline Sandy, brown clay, and caliche pebules & 3.0 & 230 \\
\hline Buff clay and fine-onained, anguiar eravel & 10 & 240 \\
\hline $\begin{array}{l}\text { Very coarse-gralned sand; andular éravel, anda } \\
\text { brown alay }\end{array}$ & 10 & 250 \\
\hline Fine to coarse-oreined, angular gravel. & 10 & 260 \\
\hline
\end{tabular}


Iable 3.--Santie loss of wells and test. boles in vicindty of MicGregcr Rariee, Oterc County, N. Mcx. - Continued 26.7 .32 .122 state of Nrow Mexico

(E1 Paso test hole 6,1953 )

$$
\text { - Continued }
$$

\begin{tabular}{|c|c|c|}
\hline Material & $\begin{array}{l}\text { Thichness } \\
\text { (feet) }\end{array}$ & $\begin{array}{l}\text { Depth } \\
\text { (feet) }\end{array}$ \\
\hline Fine to coarse-Erained sand & 10 & 270 \\
\hline Coarse-srained sand, and ancular pravel & 10 & 230 \\
\hline Fine to coarse-grained sand & 10 & 290 \\
\hline Sandy, buff clay & 10 & 300 \\
\hline Fine $t:$ coarse-grained sanả & 10 & 310 \\
\hline Coarse-grained sand, srme gravel and rinis olay & $10^{\circ}$ & 320 \\
\hline Sanay, brown clay & 10 & 330 \\
\hline Brown clay, crarse-frained sand, aria gravel & 10 & 340 \\
\hline Vory line-grained sand & 8 & $3+3$ \\
\hline Burf clay and ccarse-grained sand & 2 & 350 \\
\hline Brown clay a:d vary fine-grained sandstone & 20 & 370 \\
\hline Angular travel and very fine-frained sand & 10 & 380 \\
\hline Very rine to fine-grained sand & .10 & 390 \\
\hline Sanày, bufi clay $\quad$. & 7 & 397 \\
\hline Brown clay and bentonite (?) & 3 & 400 \\
\hline Pink clay and ccarse-grained sand & 10 & 410 \\
\hline Sandy, pink clay & 4 & 424 \\
\hline Sand and clay & 6 & 420 \\
\hline Fine to medium-rrained sand & 10 & 430 \\
\hline Fine to mediun-sralned sand, and clay & 3.0 & 4160 \\
\hline
\end{tabular}


Table 3.--Semule lous of rells and test holes ir ridintity of NicGrefor Kan!e, Otiro Comaty, N. Mex. - Continued

\section{7 .32 .122 Suate of Now Nex100}

(El Paso test nole 6, 1953)

- C intinued

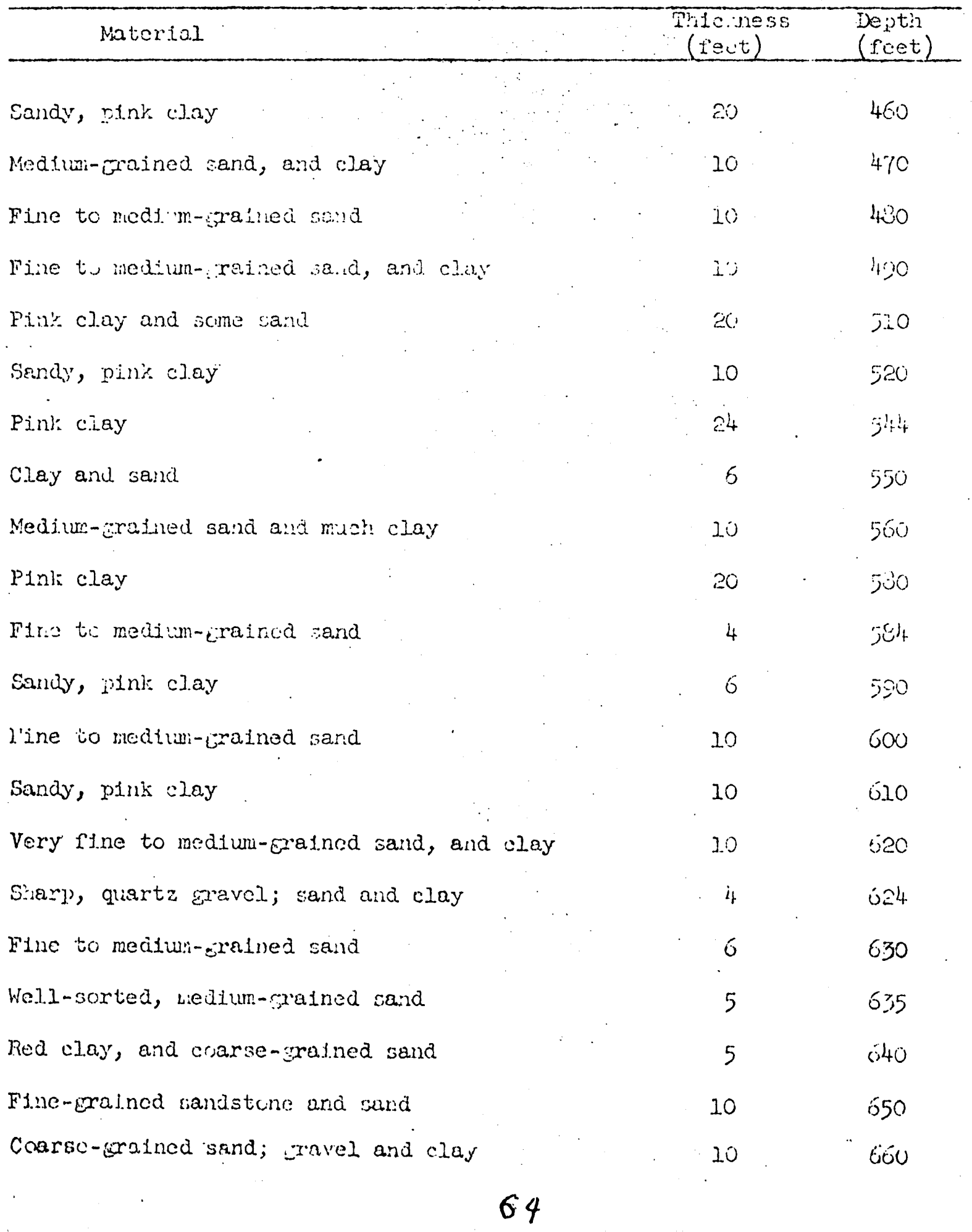


Table 3.--Sample logs of wejus and test holes in vivinity of' McGreeror Ringe, Otero County, $N$. Mex. - Cont1mued 25.7 .32 .122 State of New Mexico

(E]. Taso iest hole 6,1953$)$

- Continuea

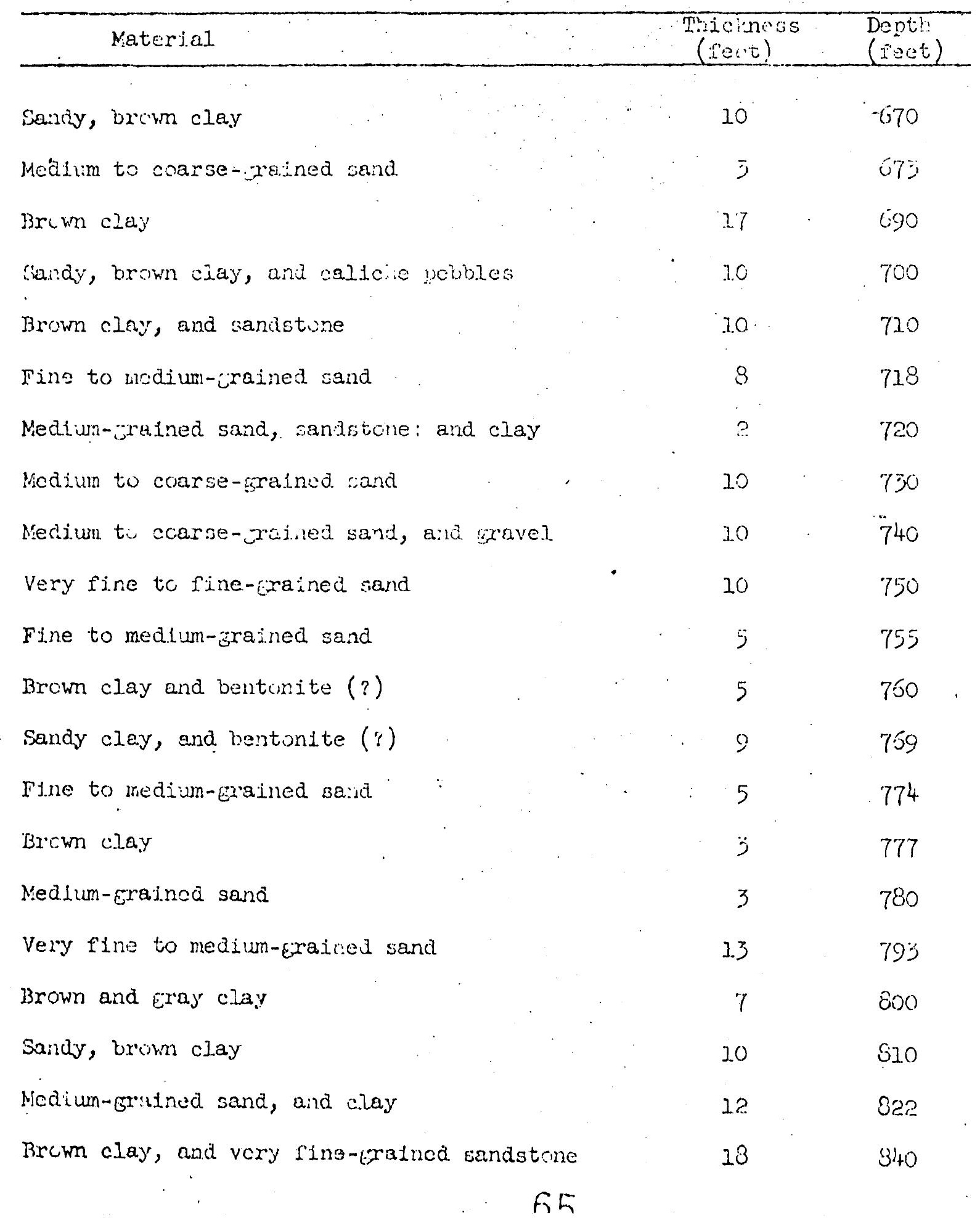


Table 3...-Sample $10, \mathrm{~s}$ of wells and test holes in vicinity of

McGiregur Range, Otero County, N. liex. - Continued

\subsubsection{State of New Vexico}

(E1. Paso tast nole 6, 2953)

- Contimued

\begin{tabular}{|c|c|c|}
\hline Material & $\begin{array}{l}\text { Tuicinuss } \\
\text { (feet) }\end{array}$ & $\begin{array}{l}\text { Depth } \\
\text { (1eet) }\end{array}$ \\
\hline$\therefore \quad \therefore \quad \cdot \quad \cdot$ & 4 & $84 t$ \\
\hline Very fine to fine-irasucd sanci & 5 & 849 \\
\hline Brown olay & .11 & 850 \\
\hline Vory fine-tratiad sandstrne, and brom riay & IU & 870 \\
\hline Vory tiale-erained sardsutine and mecium-Erained & 20 & 880 \\
\hline
\end{tabular}
sinati 
- Table 3.--Sample logs of well.s and test holes in vicinity of McGregur Range, Otaro Countj, N. Miex. - Continuea

$$
26.9 .32 .111
$$

(El Paso test 1.01e 8, 1953)

\begin{tabular}{ccc}
\hline Naterial & Thichess & $\begin{array}{c}\text { Devth } \\
\text { (feet) }\end{array}$ \\
\hline
\end{tabular}

Mediun to coarse-frahied sand, ard caliche $30 \quad 30$

Madium to course-eralred sand

$20 \quad 50$

Vory flne to f'ine-graineu sand

40

90

Very fine to medium-grained sard

20

100

Fine to coarse-crained sand

10

210

Very flne to medium-gralned sand

60

170

Very fine to very coarse-cratica sand

30

200

Vcry line to ccarse-fralned said and some clay

$\ddot{0} 0$

230

Very fine-grained sand, silt and sime ilay

20

250

Very fine to medium-crainca sand. Little clay

10

260

Very fine to medium-crainad sand, and clay

10

270

Brown clay and very fine-Grained sand

10

280

Brown c:lay

10

290

Brown clay and sume very fine-grained sand

10

300

Red clay, some silt and fine-gralned sand

10

310

Red clay and some fine-grained sand

30

340

Red clay, very fine-gralned sand and gray filt

30

370

Red clay, and medrum to coarse-erained sand

10

330

Red clay ana medium to vary coarse-frajned sand

1.0

390 
- 'able 3.--Sample lozs of' wolls and test boles in vinintty af HeGreger Rance, otero County, $N$. Hex. - Continued

\section{$20 \cdot 3 \cdot 32.111$}

(Fi) Paso tesi role $\varepsilon, 1.953$ )

$$
\text { -Conis med }
$$

\begin{tabular}{|c|c|c|}
\hline Material & $\begin{array}{l}\text { Trioleness } \\
\text { (reet) }\end{array}$ & $\begin{array}{l}\text { Diepti } \\
(f e c t)\end{array}$ \\
\hline $\begin{array}{l}\text { Mediun to very coarse-srained serid, clay and } \\
\text { some gravel. }\end{array}$ & 1.0 & 4,10 \\
\hline Mediun to very crarse-wained sand and red clay & 10 & 410 \\
\hline 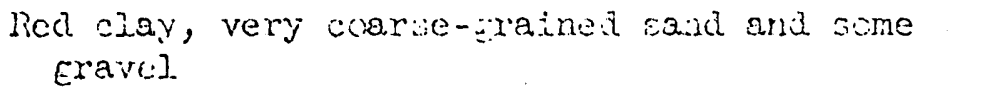 & 20 & 430 \\
\hline Rod clay and very f'ine to ciarse-rrained sand & 20 & 440 \\
\hline Red clay, cray silt a:d sume gravel & 20 & 450 \\
\hline Red clay and silt & 10 & 460 \\
\hline $\begin{array}{l}\text { Ped cley, rilt, very coarce-prained sand and } \\
\text { sonie eravel }\end{array}$ & 30 & 490 \\
\hline Red clay and silt & 10 & 500 \\
\hline Red clay, Eilt and t:in strata of callohe & 10 & 510 \\
\hline $\begin{array}{l}\text { Red clay, silt, fine caltche travel and sone } \\
\text { coarse-cralned saind }\end{array}$ & .10 & 520 \\
\hline Red clay, silt and some caliche & 20 & 540 \\
\hline $\begin{array}{l}\text { Red clay, nicdin to coarse-crained saind, silt } \\
\text { alld soine crovel }\end{array}$ & 10 & 530 \\
\hline Brown elny, silt, end medium to cuarse-urained & sand & 570 \\
\hline $\begin{array}{l}\text { Brown clay, very fjne to medium-grained sand } \\
\text { and sime cravel }\end{array}$ & 20 & 590 \\
\hline $\begin{array}{l}\text { Brown clay, very finc to ccuarue-crained sand, } \\
\text { and cravel }\end{array}$ & 1.0 & $\overline{0} 00$ \\
\hline $\begin{array}{l}\text { Red and brown sility clay, very fine to medium-. } \\
\text { grained sand, and gravel }\end{array}$ & 20 & 620 \\
\hline $\begin{array}{l}\text { Brown clay, very line to mediun-rrained sand, } \\
\text { aud cravel }\end{array}$ & 10 & 630 \\
\hline
\end{tabular}


Iable 3.--Sanple loss of wella and test holeo in vicinity of licGregor Rance, oterc coumit, N. Mex. - Continued

2.0.32.111

(E1 paso test kele 3, 1953)

- Cont lined

\begin{tabular}{|c|c|c|}
\hline Matarial & $\begin{array}{l}\text { Thicliness } \\
\text { (fect) }\end{array}$ & $\begin{array}{l}\text { Dept:? } \\
\text { (feet) }\end{array}$ \\
\hline $\begin{array}{l}\text { Irrcum clay, very fine to coarse-grained sand, } \\
\text { gravel aid fobbles }\end{array}$ & 10 & 640 \\
\hline $\begin{array}{l}\text { Very fine to very coarse-erainad sand, slay, } \\
\text { pravol and pebbles }\end{array}$ & 10 & 850 \\
\hline 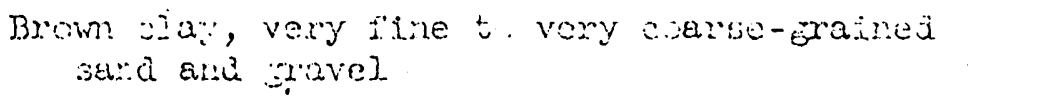 & 20 & 670 \\
\hline $\begin{array}{l}\text { Druwa clay, very fine-grained sand, gravel } \\
\text { and benbles }\end{array}$ & 10 & 680 \\
\hline 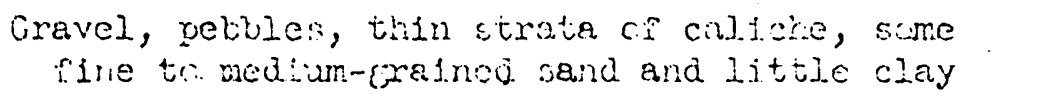 & 5 & 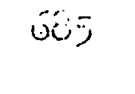 \\
\hline Gravel and pobbles & j & 690 \\
\hline $\begin{array}{l}\text { Gravel, pebbles, red clay and sme very fine to } \\
\text { medían-crained sand }\end{array}$ & 3.0 & 700 \\
\hline 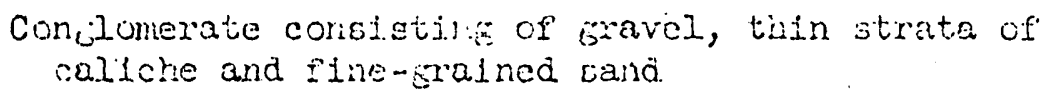 & 30 & 730 \\
\hline $\begin{array}{c}\text { Conslomerate of Eravel, pebblee, and fine-grifned } \\
\operatorname{sana} \text { : }\end{array}$ & .10 & $7^{4}+0$ \\
\hline Conclumerate of gravel and pebbles & 40 & 780 \\
\hline $\begin{array}{l}\text { Conglonerate of gravel, pobbles, some red clay } \\
\text { and very fine-guined sard }\end{array}$ & 30 & 810 \\
\hline $\begin{array}{l}\text { Conglumerate of gravel, pebries and very tine to } \\
\text { ciarse-jrahned said }\end{array}$ & 10 & 820 \\
\hline
\end{tabular}


"'able 4..-Driller's log of well in viciuity of liscrefor Rance, otero County, New Mexico. $26.6 .34 .230 \mathrm{a}$ Southern Pacifie Lines (We.21. 3) (We1.1 140 in water supi]y Paner 919)

\begin{tabular}{|c|c|c|c|c|c|c|c|c|}
\hline & Matortal & & & . & & & $\begin{array}{l}\text { Tincloness } \\
\text { (fect) }\end{array}$ & $\begin{array}{l}\text { Depth } \\
\text { (reet) }\end{array}$ \\
\hline Sand & & & & & & & 20 & 10 \\
\hline Calich & & & & & ." & & 5 & $\cdots 15$ \\
\hline Sand & & & & & & & 135 & 150 \\
\hline Clay & & & & & . & & 5 & $15 \%$ \\
\hline Sand & & & & & & & 20 & 175 \\
\hline Clay & & & & & & & .4 & 179 \\
\hline Sand & & . & & & & & 8 & 187 \\
\hline Clay & $\cdot$ & & & & & & 3 & 190 \\
\hline $\operatorname{San} \hat{a}$ & & & & & . & & 43 & 233 \\
\hline ciay & & & & & & & it & 237 \\
\hline Sand & & & & & & & 7 & 244 \\
\hline Rock & & & & & & & 1 & $21+5$ \\
\hline $\operatorname{sand}$ & & . & & & & . & 55 & $300^{-}$ \\
\hline Clay & & & & & & & 4 & 304 \\
\hline $\operatorname{San} \bar{a}$ & & . : : & $\therefore$ & $\therefore$ & & & 4 & 308 \\
\hline Clay & & & & . & & . & 4 & 312 \\
\hline Sand, & water & & & . & & & 38 & 350 \\
\hline clay & & - & & & & & 10 & 360 \\
\hline Saridst & one & & & & & & 20 & 380 \\
\hline
\end{tabular}


Table 4.-.Driller's.10is of well in vieinity of NicGrogor Ranee, Otero County, New Mexico - Continued

$26.6 .34 .130 \mathrm{a}$ Soithern Paciffe Lines (Woll 3)

(Well 140 in Water Suiply Paner 919)

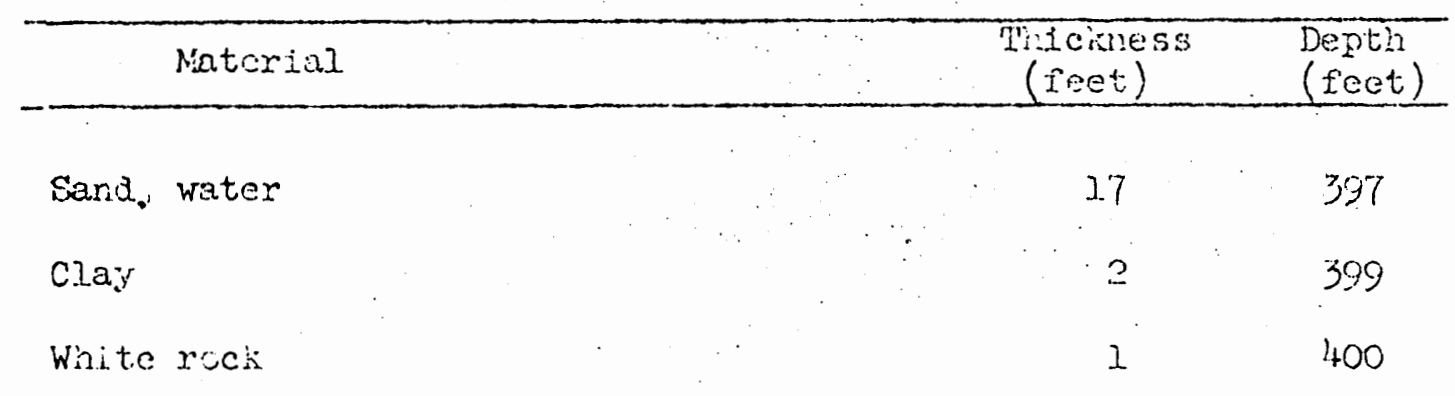


[lable 5.--Driler's log of well in vicinity (r MeGrecor Rance, El Paso Cunty, Texas

1.1.6.340 Iravar Bros.

(Partial log)

\begin{tabular}{|c|c|c|}
\hline liaterial & $\begin{array}{l}\text { Thismers } \\
\text { (sent) }\end{array}$ & $\begin{array}{l}\text { Derti2 } \\
\text { (reat) }\end{array}$ \\
\hline Clay and ssund streaks; very littile sand & $\therefore 4010$ & 400 \\
\hline Coniclomerate and yellow sune & 35 & $4 \dddot{35}$ \\
\hline Conglomerate & 24 & 459 \\
\hline 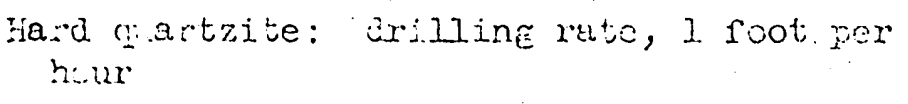 & $?$ & $!+\because{ }^{\circ}$ \\
\hline Blaci: siale, and lime & 12 & 10 \\
\hline Iitie & 5 & 4.65 \\
\hline Llmc and sinale strears & .27 & 502 \\
\hline Soft, blasli siln?e. & 12 & 514 \\
\hline
\end{tabular}

Well is be aring a to 600 fret. 


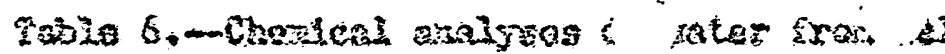
Gero Gon

(f)

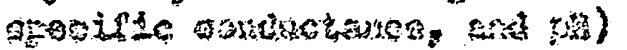

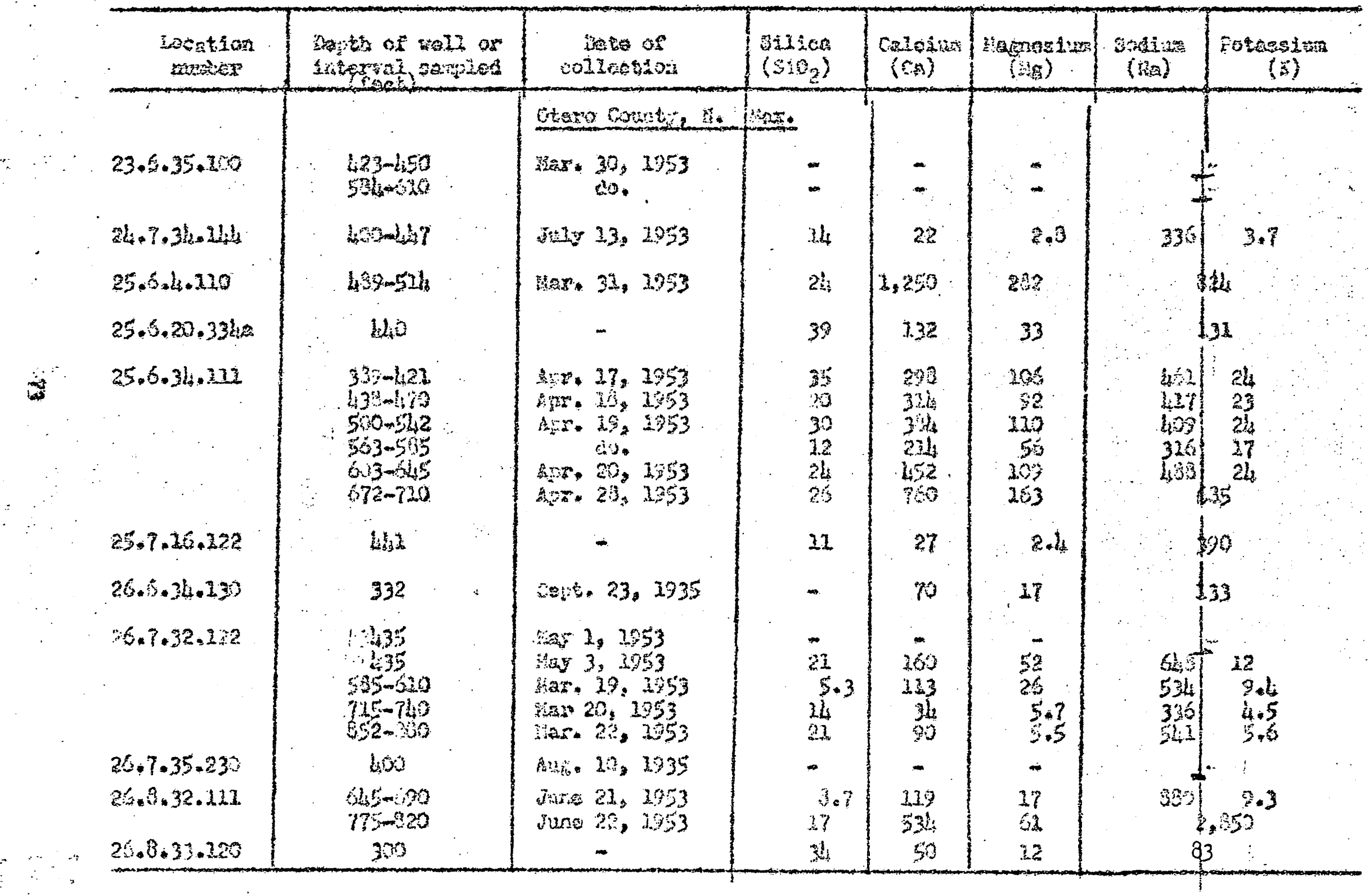


Table 6.-Conical analgses of wet from well ma otero County, H. Fiex.; ad $\mathrm{El}$ Paso vount tholes in the vicinity of Koúregor Range; Ter. Costis. 2

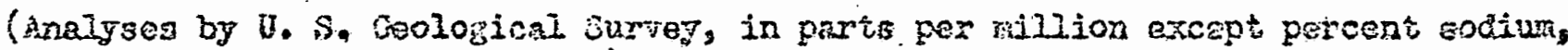
spocinic conductance, and pil

\begin{tabular}{|c|c|c|c|c|c|c|c|c|c|c|c|c|}
\hline \multirow{2}{*}{$\begin{array}{l}\text { Iscation } \\
\text { number }\end{array}$} & \multirow{2}{*}{$\begin{array}{l}\text { ELCar- } \\
\text { Soneto } \\
\left(\mathrm{HCO}_{3}\right)\end{array}$} & \multirow{2}{*}{$\begin{array}{c}\text { Sulfate } \\
\left(\mathrm{So}_{4}\right)\end{array}$} & \multirow{2}{*}{$\frac{\operatorname{mar} 20}{(0 I)}$} & \multirow{2}{*}{$\underset{(E)}{\text { Eluoride }}$} & \multirow{2}{*}{$\left|\begin{array}{ll}\text { Pritrate } \\
\left(\mathrm{MO}_{3}\right)\end{array}\right|$} & \multirow{2}{*}{$\begin{array}{c}\text { Boron } \\
\text { (B) }\end{array}$} & \multirow{2}{*}{$\begin{array}{c}\text { Dissolved } \\
\text { solids }\end{array}$} & \multirow{2}{*}{\multicolumn{2}{|c|}{$\frac{\text { heringss } 2.5 \mathrm{CaCO}_{3}}{\text { Total foumsarbonate }}$}} & \multirow{2}{*}{$\begin{array}{l}\text { Yercen } \\
\text { sodiun } \\
(\mathrm{N} / \mathrm{Na})\end{array}$} & \multirow{2}{*}{$\begin{array}{l}\text { Specjific } \\
\text { conductance } \\
(\text { minconios } \\
\left.\text { et } 25^{\circ} \mathrm{C}\right)\end{array}$} & \multirow{2}{*}{ pin } \\
\hline & & & & & & & & & & & & \\
\hline 23.5 .35 .200 & 47 & 1,470 & 6.720 & - & oter & Count & y, hen Hox & $\frac{1}{17.950}$ & - & - & 39.000 & 6.5 \\
\hline & 35 & 2,460 & 8,010 & - & - & - & - & 8,950 & - & - & 22,500 & 6.6 \\
\hline $24 \cdot 7 \cdot 34 \cdot 14 h_{4}$ & 96 & 387 & 232 & 1.1 & 0.5 & 0.34 & 1,050 & 6 & 0 & 92 & 2,800 & 7.7 \\
\hline 25.6 .4 .120 & 36 & 1,210 & 3,390 & .0 & - & - & 7,000 & 4,200 & 4,250 & 30 & 11,000 & 6.7 \\
\hline $85 \cdot 6 \cdot 20 \cdot 334 \mathrm{~A}$ & SI & 50 & 4,33 & .4 & 6.3 & - & 375 & 4,65 & 390 & - & 1,640 & 7.5 \\
\hline 25.5 .34 .111 & $\begin{array}{l}92 \\
96 \\
53 \\
69 \\
49 \\
35\end{array}$ & $\begin{array}{l}779 \\
571 \\
663 \\
410 \\
212 \\
212\end{array}$ & $\begin{array}{r}940 \\
1,020 \\
1,120 \\
720 \\
1,720 \\
2,700\end{array}$ & $\begin{array}{l}.4 \\
.4 \\
.6 \\
.2 \\
.0\end{array}$ & $\begin{array}{l}4.2 \\
3.5 \\
2.0 \\
.2 \\
1.0 \\
-\end{array}$ & $\begin{array}{l}.25 \\
.29 \\
.15 \\
.26 \\
.23 \\
-\end{array}$ & $\begin{array}{l}2,690 \\
2,510 \\
2,770 \\
1,780 \\
3,050 \\
4,560\end{array}$ & $\begin{array}{l}1,150 \\
2,150 \\
1,4,10 \\
754 \\
1,500 \\
2,570\end{array}$ & $\begin{array}{r}1,100 \\
1,030 \\
1,370 \\
700 \\
1,540 \\
2,540\end{array}$ & $\begin{array}{l}45 \\
43 \\
33 \\
47 \\
40 \\
37\end{array}$ & $\begin{array}{l}4,150 \\
1,330 \\
4,470 \\
3,230 \\
5,490 \\
5,360\end{array}$ & $\begin{array}{l}7.5 \\
7.4 \\
7.3 \\
7.2 \\
7.2 \\
7.1\end{array}$ \\
\hline 25.7 .16 .122 & 76 & 310 & 380 & .9 & 2.0 & - & 1,160 & 78 & 15 & - & 2,350 & 7.5 \\
\hline 26.6 .34 .130 & 98 & 30 & 290 & - & 5.2 & - & 602 & 24.5 & - & - & - & - \\
\hline $25,7.32 .322$ & $\begin{array}{l}113 \\
36 \\
59 \\
81 \\
47\end{array}$ & $\begin{array}{l}-7 \\
602 \\
622 \\
70 \\
44\end{array}$ & $\begin{array}{l}720 \\
710 \\
620 \\
505 \\
950\end{array}$ & $\begin{array}{r}-.5 \\
.5 \\
.3 \\
.7 \\
1.0\end{array}$ & $\begin{array}{r}1.0 \\
.0 \\
.0 \\
.0\end{array}$ & $\begin{array}{l}- \\
.24 \\
.22 \\
.35 \\
.35\end{array}$ & $\begin{array}{l}2,550 \\
1,950 \\
1,010 \\
1,600\end{array}$ & $\begin{array}{l}58 \\
523 \\
339 \\
108 \\
247\end{array}$ & $\begin{array}{l}54.2 \\
340 \\
42 \\
208\end{array}$ & $\begin{array}{l}69 \\
74 \\
85 \\
82\end{array}$ & $\begin{array}{l}3,370 \\
3,890 \\
3,220 \\
1,930 \\
3,220\end{array}$ & $\begin{array}{l}7.6 \\
7.5 \\
7.3 \\
7.4 \\
7.3\end{array}$ \\
\hline $26.7 \cdot 35.230$ & 176 & - & 91 & - & - & - & - & 33 & - & - & - & - \\
\hline $26.8 \cdot 32.111$ & $\begin{array}{l}53 \\
62\end{array}$ & $\frac{163}{6.11}$ & $\begin{array}{l}2,450 \\
5,030\end{array}$ & .9 & .5 & .33 & $\begin{array}{l}2,670 \\
8,130\end{array}$ & $\begin{array}{r}367 \\
1,530\end{array}$ & $\begin{array}{l}324 \\
1530\end{array}$ & $\begin{array}{l}83 \\
30\end{array}$ & $\begin{array}{r}4,300 \\
15,100\end{array}$ & $\begin{array}{l}7.4 \\
7.3\end{array}$ \\
\hline 25.9 .33 .120 & 134 & 1.36 & 41 & .5 & 54 & $=$ & 435 & 174 & 64 & - & 725 & 3.1 \\
\hline
\end{tabular}


Tabie 6.-Chemical enalyses of ter from $a$ a test holes in the vicinity of wecregor Ran

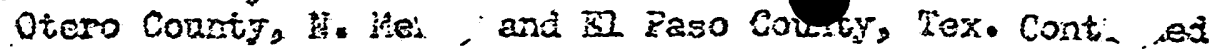

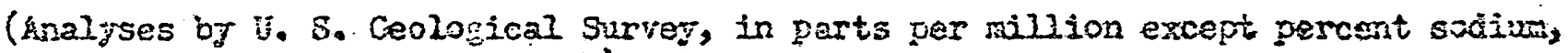
sFecific conductarce, and $\mathrm{pLS}$ )

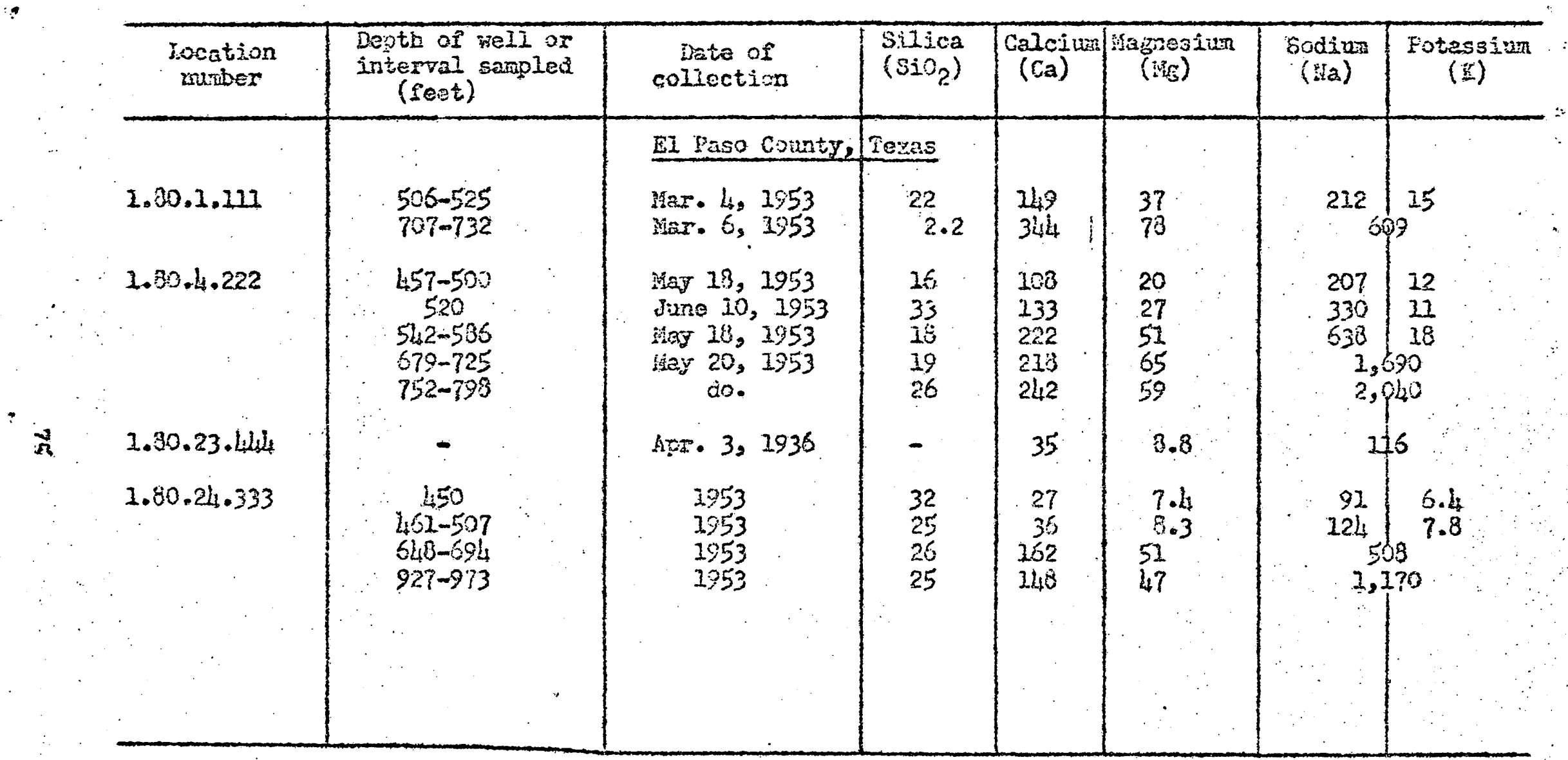




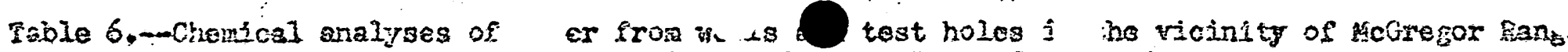

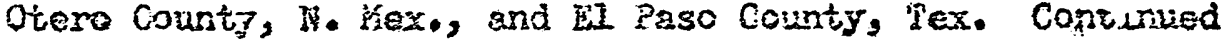

(Analysos by U. 5. Gological Survey, in parts per million except percent soajun, spocifie coiductance, and pij)

\begin{tabular}{|c|c|c|c|c|c|c|c|c|c|c|c|c|}
\hline \multirow{2}{*}{$\begin{array}{l}\text { Location } \\
\text { number }\end{array}$} & \multirow{2}{*}{$\begin{array}{l}\text { Dicar- } \\
\text { bonate } \\
\left(\mathrm{HCO}_{3}\right)\end{array}$} & \multirow{2}{*}{$\frac{(5 \mathrm{a}) \mathrm{fate}}{\left(5 \mathrm{O}_{4}\right)}$} & \multirow{2}{*}{$\frac{\text { buroride }}{(c 1)}$} & \multirow{2}{*}{$\frac{\text { Mueride }}{(n)}$} & \multirow{2}{*}{$\left(\begin{array}{c}\text { itrate } \\
\left(\mathrm{m}_{3}\right)\end{array}\right.$} & \multirow{2}{*}{$\begin{array}{c}\text { Boron } \\
(B)\end{array}$} & \multirow{2}{*}{$\begin{array}{r}\text { Disoudyed } \\
\text { solids }\end{array}$} & \multicolumn{2}{|c|}{ Mardness as $\mathrm{CaCO}_{3}$} & \multirow{2}{*}{ 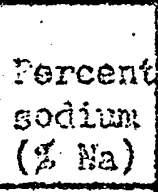 } & \multirow{2}{*}{ 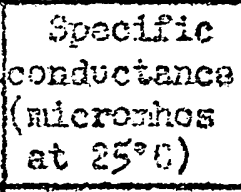 } & \multirow{2}{*}{ pi } \\
\hline & & & & & & & & Tosal & Hioncarbonato & & & \\
\hline & & & - & & 19 & $80 \mathrm{Cos}$ & ty, iexes & & & & & \\
\hline .80 .2 .111 & $\begin{array}{l}72 \\
37\end{array}$ & $\begin{array}{l}70 \\
72\end{array}$ & $\begin{array}{r}632 \\
1,700\end{array}$ & 0.5 & $\begin{array}{r}4.0 \\
.5\end{array}$ & 0.36 & $\begin{array}{l}1,360 \\
2,320\end{array}$ & $\begin{array}{r}524 \\
1,130\end{array}$ & $\begin{array}{r}465 \\
1,150\end{array}$ & $\begin{array}{l}45 \\
53\end{array}$ & $\begin{array}{l}2,180 \\
5,160\end{array}$ & $\begin{array}{l}7.5 \\
7.2\end{array}$ \\
\hline .80 .4 .222 & $\begin{array}{r}91 \\
60 \\
64 \\
94 \\
163\end{array}$ & $\begin{array}{r}36 \\
11 \\
25 \\
233 \\
627\end{array}$ & $\begin{array}{r}492 \\
795 \\
1,510 \\
2,950 \\
3,20\end{array}$ & $\begin{array}{l}.3 \\
.0 \\
.0 \\
.7 \\
.6\end{array}$ & $\begin{array}{l}5.3 \\
5.4 \\
3.0 \\
- \\
-\end{array}$ & $\begin{array}{l}.16 \\
.09 \\
.09 \\
- \\
-\end{array}$ & $\begin{array}{r}943 \\
1,330 \\
2,520 \\
5,220 \\
6,260\end{array}$ & $\begin{array}{l}352 \\
443 \\
764 \\
712 \\
846\end{array}$ & $\begin{array}{l}277 \\
394 \\
717 \\
734 \\
713\end{array}$ & $\begin{array}{l}55 \\
61 \\
64 \\
82 \\
84\end{array}$ & $\begin{array}{r}1,550 \\
2,700 \\
4,350 \\
9,260 \\
10,500\end{array}$ & $\begin{array}{l}7.2 \\
7.5 \\
7.3 \\
7.0 \\
7.0\end{array}$ \\
\hline .00 .23 .444 & 146 & 54 & 139 & - & 5.0 & - & 430 & 124 & - & - & - & - \\
\hline .20 .24 .333 & $\begin{array}{c}151 \\
133 \\
57 \\
86\end{array}$ & $\begin{array}{r}53 \\
67 \\
48 \\
137\end{array}$ & $\begin{array}{r}39 \\
158 \\
1,350 \\
2,040\end{array}$ & $\begin{array}{l}.5 \\
.6 \\
.2 \\
.6\end{array}$ & $\begin{array}{c}5.0 \\
4.5 \\
.0 \\
-\end{array}$ & $\begin{array}{l}.16 \\
.25 \\
- \\
-\end{array}$ & $\begin{array}{r}336 \\
493 \\
2,970 \\
3,610\end{array}$ & $\begin{array}{l}93 \\
124 \\
614 \\
563\end{array}$ & $\begin{array}{r}0 \\
15 \\
567 \\
404\end{array}$ & $\begin{array}{l}- \\
-\end{array}$ & $\begin{array}{r}643 \\
365 \\
3,960 \\
6,560\end{array}$ & $\begin{array}{l}7.6 \\
7.9 \\
7.2 \\
7.1\end{array}$ \\
\hline
\end{tabular}

